\title{
WestVirginiaUniversity
}

THE RESEARCH REPOSITORY @ WVU

Graduate Theses, Dissertations, and Problem Reports

2006

\section{Magnetic and structural properties of nanoparticles of nickel oxide}

Hyunja (Jenny) Shim

West Virginia University

Follow this and additional works at: https://researchrepository.wvu.edu/etd

\section{Recommended Citation}

Shim, Hyunja (Jenny), "Magnetic and structural properties of nanoparticles of nickel oxide" (2006). Graduate Theses, Dissertations, and Problem Reports. 2421.

https://researchrepository.wvu.edu/etd/2421

This Dissertation is protected by copyright and/or related rights. It has been brought to you by the The Research Repository @ WVU with permission from the rights-holder(s). You are free to use this Dissertation in any way that is permitted by the copyright and related rights legislation that applies to your use. For other uses you must obtain permission from the rights-holder(s) directly, unless additional rights are indicated by a Creative Commons license in the record and/ or on the work itself. This Dissertation has been accepted for inclusion in WVU Graduate Theses, Dissertations, and Problem Reports collection by an authorized administrator of The Research Repository @ WVU.

For more information, please contact researchrepository@mail.wvu.edu. 


\section{Magnetic and Structural Properties of Nanoparticles of Nickel Oxide}

By Hyunja (Jenny) Shim

\section{DISSERTATION}

Submitted to the Eberly College of Arts and Sciences

at

West Virginia University

In Partial Fulfillment of the Requirements for the Degree of

Doctor of Philosophy

in Physics

Mohindar S. Seehra, Ph.D., Chair

Larry E. Halliburton, Ph.D.

Lloyd Carroll, Ph.D.

Martina E. Bachlechner, Ph.D.

Sergei Urazhdin, Ph.D.

Morgantown, West Virginia

2006

Keywords: Nickel Oxide, Nanoparticles, Magnetism

Copyright 2006 Hyunja Shim 
ABSTRACT

Magnetic and Structural Properties of Nanoparticles of Nickel Oxide

By Hyunja (Jenny) Shim

In this dissertation, magnetic properties of $\mathrm{NiO}$ nanoparticles (NP) prepared by the sol-gel method in the size range $\mathrm{D}=5 \mathrm{~nm}$ to $20 \mathrm{~nm}$, with and without oleic acid (OA) coating, are reported. Transmission electron microscopy (TEM) studies show the morphology of the smaller particles to be primarily rod-like, changing over to nearly spherical shapes for $\mathrm{D}>10 \mathrm{~nm}$. Average sizes D of NP determined by x-ray diffraction (XRD) are compared with the results from TEM. From the analysis of the XRD line intensities, the particle size dependence of the Debye-Waller factors for $\mathrm{Ni}$ and $\mathrm{O}$ atoms are derived. It is found that the Debye-Waller factors of nickel and oxygen atoms in smaller particles are larger than those in bulk NiO.

For the coated and uncoated $\mathrm{NiO}$ nanorods of $5 \mathrm{~nm}$ diameter, variations of the magnetization $\mathrm{M}$ with temperature $\mathrm{T}(5 \mathrm{~K}$ to $370 \mathrm{~K})$ and temperature variations of the EMR (electron magnetic resonance) spectra were measured to determine the respective blocking temperatures $T_{B}(m)$ and $T_{B}(E M R)$. The following differences are noted: (1) $\mathrm{T}_{\mathrm{B}}(\mathrm{m})$ is reduced from $230 \mathrm{~K}$ (uncoated) to $85 \mathrm{~K}$ (coated) for $\mathrm{H}=25$ Oe; (2) Decrease of $T_{B}(m)$ with $H$ is weaker and the ratio $T_{B}(E M R) / T_{B}(m)$ is smaller for the uncoated particles. These differences are due to stronger interparticle interaction present in the uncoated particles.

Temperature variation $(5 \mathrm{~K}-300 \mathrm{~K})$ of the $\mathrm{AC}$ magnetic susceptibilities ( $\chi^{\prime}$ and $\left.\chi^{\prime \prime}\right)$ at various frequencies $\mathrm{f}(0.1-10,000 \mathrm{~Hz})$ are reported for the coated and uncoated $5 \mathrm{~nm}$ diameter nanorods of $\mathrm{NiO}$. Using the peak in $\chi^{\prime}$ as the blocking temperature $\mathrm{T}_{\mathrm{B}}$, it is observed that $T_{B}$ increases with increasing $f$. The data for the two samples fit the VogelFulcher law: $\mathrm{f}=\mathrm{f}_{0} \exp \left[-\mathrm{E}_{\mathrm{a}} / \mathrm{k}\left(\mathrm{T}_{\mathrm{B}}-\mathrm{T}_{0}\right)\right]$ with $\mathrm{f}_{0}=9.2 \times 10^{11} \mathrm{~Hz}, \mathrm{E}_{\mathrm{a}} / \mathrm{k}=1085 \mathrm{~K}$ and $\mathrm{T}_{0}=162$ $\mathrm{K}(0 \mathrm{~K})$ for the uncoated (coated) particles. This shows that $\mathrm{T}_{0}$ provides a good measure of the effects of interparticle interactions on magnetic relaxation and that these interactions are essentially eliminated with the OA coating.

For all the particles, measurements of $\mathrm{M}$ versus $\mathrm{T}(5 \mathrm{~K}-370 \mathrm{~K})$ in the zero-field cooled (ZFC) and field-cooled (FC) modes are used to determine the average blocking temperature $T_{P}$. For the OA coated particles, $T_{P}$ increases with increase in size $D$ as expected for superparamagnetic particles. However for the uncoated NP, $T_{P}$ decreases initially with increase in size for $\mathrm{D}<10 \mathrm{~nm}$; but for $\mathrm{D}>10 \mathrm{~nm}, \mathrm{~T}_{\mathrm{P}}$ follows the same 
trend as for the coated NP. These differences are interpreted in terms of significant interparticle interaction. The data of $\mathrm{M}$ vs. the applied field $\mathrm{H}$ for $\mathrm{T}>\mathrm{T}_{\mathrm{P}}$ are fit to the modified Langevin function: $\mathrm{M}=\mathrm{M}_{0} \mathscr{L}\left(\mu_{\mathrm{P}} \mathrm{H} / \mathrm{k}_{\mathrm{B}} \mathrm{T}\right)+\chi_{\mathrm{a}} \mathrm{H}$, to determine the magnetic moment $\mu_{\mathrm{P}}$ per particle as a function of size $\mathrm{D}$. The variation of $\mu_{\mathrm{P}}$ with size $\mathrm{D}$ is interpreted in terms of the fraction of spins on the surface layer of the particles which contribute to $\mu_{\mathrm{P}}$. It is observed that this fraction varies as $1 / \mathrm{D}$ reaching nearly $100 \%$ for the $5 \mathrm{~nm}$ particles. From the temperature dependence of $\mathrm{M}_{0}$ and extrapolating to $\mathrm{M}_{0} \rightarrow 0$, the Néel temperatures $T_{N}$ for various sizes are determined. $T_{N}$ for $\mathrm{NiO}$ nanoparticles is found to decreases rapidly with decrease in size for $\mathrm{D}<10 \mathrm{~nm}$. 


\section{ACKNOWLEDGEMENTS}

It is my pleasure to acknowledge the following people for their invaluable support during my years of study at West Virginia University.

First, I would like express my sincere gratitude and many thanks to my research advisor, Prof. M.S. Seehra, for suggesting the problem, for his fruitful guidance, assistance and encouragement throughout my research project and for his assistance in personal matters.

Thanks to each of my committee members, Dr. Larry E. Halliburton, Dr. Martina Bachlechner, Dr. Sergei Urazhdin, and Dr. Lloyd Carroll for spending time out of their insanely busy schedules to fit me in so I could finish.

I wish to thank Dr. A. Manivannan for his generous help in the X-ray diffraction, TEM, and SQUID measurements. I also wish to thank Dr. Nancy Giles, Dr. Thomas H. Myers, Dr. David Lederman and Dr. Art Weldon for their wonderful lectures and generosity with their time. I thank Dr. J. Bonevich for carrying out the TEM studies reported in this work. My gratitude and sincere thanks to Dr. Jagadeesh S. Moodera, Dr. Wooyoung Lee, Dr. Jung -Bum Choi, and Dr. Yang-Ki Hong, for their fruitful advice.

My sincere thanks goes to Prof. Seehra's post-doctoral associates Dr. Prasant Dutta (2003-present) and Dr. Alex Punnoose (2002) for their professional help and friendship and Dr. Halliburton's post-doctoral associate, Dr. Nelson for his professional help in computer programming. I wish to thank the people in my lab, Aashish, Vivek, Sukanaya, Susmita, and Jeremy. My special thanks to Siobhan, Sherry, and Sandy for their friendship and timely help during various occasions. Technical assistance provided by Phil and Tom is greatly acknowledged. I wish to thank my colleagues, Chunchuan, Jorge, Zhang, and everyone else I have forgotten to mention. I express my sincere thanks to my friends, Sungho, Dr. Yang and his family, Pastor Kim and his family. I also wish to acknowledge, my aunt and her family in Chicago, my relatives, uncles, aunts and their family, grand mother and grand father in Korea.

Finally, I have a deep sense of gratitude towards my mother for her constant support and understanding. I would like to thank my brothers, Youngyoon and Youngjun for their invaluable understanding and encouragement. 


\section{TABLE OF CONTENTS}

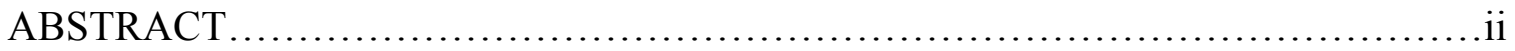

ACKNOWLEDGEMENTS ................................................................... iv

LIST OF FIGURES................................................................

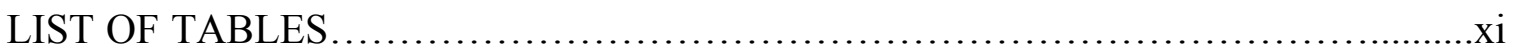

I. INTRODUCTION

1.1 Magnetic properties of Bulk NiO.....................................

1.2 Magnetic properties of $\mathrm{NiO}$ Nanoparticles.................................1

1.3 Motivation for the present work......................................

II. MOLECULAR FIELD THEORY OF MAGNETIC ORDERING

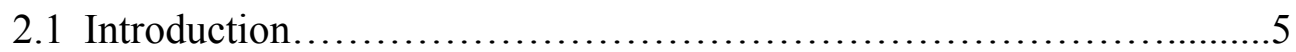

2.2 The secular equation.........................................6

2.3 Three types of ordering and equations for $\mathrm{T}_{\mathrm{N}}$ and $\theta \ldots \ldots \ldots \ldots \ldots \ldots 11$

2.4 The $\mathrm{J}_{1}-\mathrm{J}_{2}$ diagram for FCC systems.................................. 14

$2.5 \mathrm{~J}_{1}$ and $\mathrm{J}_{2}$ for the FCC system.................................... 16

2.6 Results of the Green's Function Theory for $J_{1}$ and $J_{2} \ldots \ldots \ldots \ldots \ldots \ldots$

2.7 Measurement of $\mathrm{J}_{1}$ and $\mathrm{J}_{2}$ for $\mathrm{NiO}$ by other methods..................22

III. SYNTHESIS AND STRUCTUREAL PROPERTIES

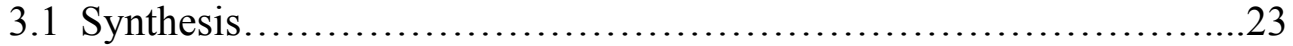

3.2 Transmission Electron Microscopy (TEM) .........................24

3.3 X-ray Diffraction (XRD) .......................................... 31

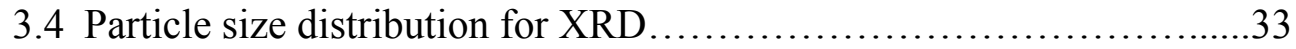

3.5 Structure Factor for NiO..........................................42

IV. REVIEW OF SUPERPARAMAGNETISM

4.1 Superparamagnetic Particles.....................................50

4.2 Critical size for single domain particles in ferromagnets..............56

4.3 Superparamagnetism in Antiferromagnetic Nanoparticles...............57

4.4 The behavior of M vs. H (Stoner-Wohlfarth Model)...................60

4.5 Relaxation and Magnetic Viscosity ...............................62 
V. EXPERIMENTAL RESULTS IN 5 NM NiO NANOPARTICLES

5.1 Electron Magnetic Resonance $($ EMR) ..........................65

5.2 Temperature Variation of the low-field magnetic susceptibility........69

5.3 Field Dependence of Blocking Temperatures........................70

5.4 Magnetization M vs. Field H.................................77

5.5 Frequency Dependence of Blocking Temperatures..................81

VI. SIZE DEPENDENCE OF THE MAGNETIC PROPERTIES IN NiO NANOPARTICLES

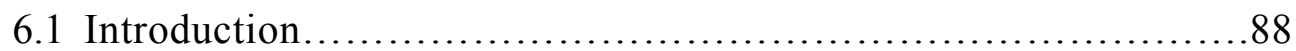

6.2 Magnetization vs. Temperature...............................89

6.3 Magnetization vs. Magnetic Field...............................94

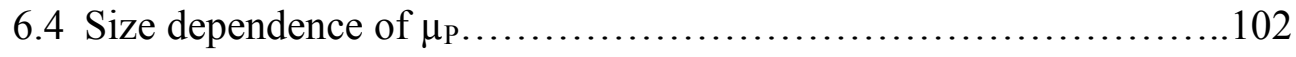

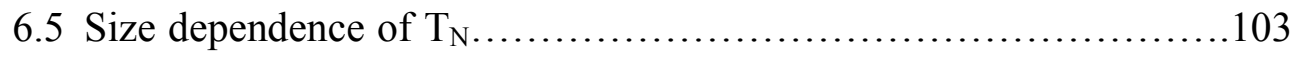

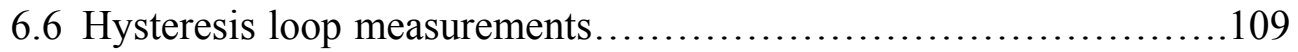

VII. CONCLUSIONS

7.1 Structure and Morphology of NiO-NP ..........................114

7.2 Effects of interparticle interaction on $5 \mathrm{~nm} \mathrm{NiO-NP \ldots \ldots \ldots \ldots ........114}$

7.3 Size dependence of the magnetic properties of NiO-NP............115

7.4 Hysteresis loop measurements of NiO-NP........................115

REFERENCES........................................................... 116 


\section{List of Figures}

Fig. 2.1 The face-centered cubic lattice divided into eight sublattices. ............7

Fig. 2.2 Antiferromagnetic types in FCC lattice. ..............................12

Fig. 2.3 Phase diagram of FCC systems with $\mathrm{J}_{1}$ and $\mathrm{J}_{2}$ [Seehra et al, 1988]......15

Fig. 2.4 The relation between $\mathrm{k}_{\mathrm{B}} \mathrm{T}_{\mathrm{N}} / \mathrm{J}_{2}$ and $\mathrm{J}_{1} / \mathrm{J}_{2}$ calculated by Green's function theory for the FCC antiferromagnetic ordering of type II and the $\mathrm{S}=1$ case [Bartel et al, 1971].

Fig. 3.1 TEM pictures of particle size D $=5 \mathrm{~nm}$ for uncoated (a) and coated (b) cases.

Fig. 3.2 Transmission electron microscopy (TEM) images of $\mathrm{NiO}(7,8,12,1620$ and $41 \mathrm{~nm}$ samples) represented in (a), (b), (c), (d), (e) and (f)

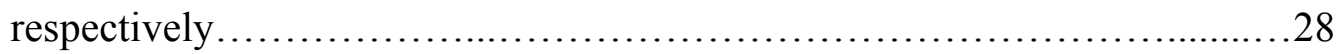

Fig. 3.3 Transmission electron microscopy (TEM) images for coated 7, 8, 12, and $20 \mathrm{~nm}$ samples represented in (a), (b), (c) and (d) respectively. 30

Fig. 3.4 XRD patterns for all particle sizes annealed at various temperatures for uncoated and coated samples. .32

Fig. 3.5 The diffracted beam for actual case (a) and ideal case (b)......34

Fig. 3.6 Diagram shows the effect of crystal size on diffraction. [Klug et al].....34

Fig. 3.7 The comparison of the XRD patterns broadening for the coated and uncoated $5 \mathrm{~nm}$ particles.

Fig. 3.8 Plots of $\beta \cos \theta$ vs. $\sin \theta$ for different samples annealed at the various temperatures and the analysis for the uncoated and coated particles............38

Fig. 3.9 Plot of the strain $\eta$ versus particle size for the coated and uncoated particles

Fig. 3.10 Plot of $\mathrm{Q}=(\mathrm{I} / \mathrm{mLP})^{1 / 2}$ vs. $\sin \theta / \lambda$ for $\mathrm{hkl}$ all even and odd each.........45

Fig. 3.11 Plots of $\ln \mathrm{Q}^{+}\left(\ln \mathrm{Q}^{-}\right)$against $(\sin \theta / \lambda)^{2}$, the slopes give directly the values of $\mathrm{B}_{1}(\mathrm{Ni})$ and $\mathrm{B}_{2}(\mathrm{O})$.

Fig. 3.12 Plots of Debye-Waller factor vs. D using the results from Table 3.4.....49

Fig. 4.1 This diagram shows the prolate ellipsoidal particle with an applied magnetic field $\mathrm{H}$ 
Fig. 4.2 For a single particle, the energy with an angle, $\alpha$ is separated by the energy barrier $1 / 2 \mathrm{CV}$ at $\alpha=\pi / 2$ between the two equivalent states at $\alpha=0$ and $\alpha=$

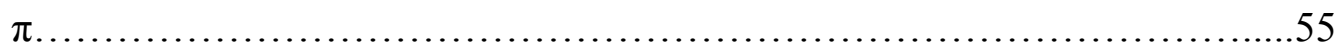

Fig. 4.3 Origin of particle magnetic moments for antiferromagnetic NiO particles proposed by Néel..................................................59

Fig. 4.4 The case of a field applied perpendicular to the polar axis. There is a reversible rotation leading to no hysteresis, and (b) is the case of that of an applied field parallel to the polar axis. No reversible rotation occurs....61

Fig. 4.5 Hysteresis curves of randomly oriented particles calculated by Stoner and Wohlfarth [Stoner et al. 1948].

Fig. 5.1 Plots of the EMR derivative line for several temperatures for the uncoated $\mathrm{NiO}$

Fig. 5.2 Plots of the resonance field $\mathrm{H}_{\mathrm{r}}$, linewidth $\Delta \mathrm{H}$, and intensity $\mathrm{I}_{0}$ of the EMR line against temperature for the coated and uncoated $\mathrm{NiO}$. .68

Fig. 5.3 Temperature dependence of the magnetic susceptibility for FC (fieldcooled) and ZFC (zero-field cooled) cases showing reduction in blocking temperature by coating. .71

Fig. 5.4 Plot of $\chi / \chi_{\max }\left(\chi_{\max }\right.$ is the value at $\mathrm{T}=\mathrm{T}_{\mathrm{B}}$ defined by the maximum) in various applied fields against temperature for coated and uncoated NP.......72

Fig. 5.5 Plots of the blocking temperature $\mathrm{T}_{\mathrm{B}}(\mathrm{H})$ at a magnetic field $\mathrm{H}$ normalized by its value at $\mathrm{H}=25 \mathrm{Oe}$ vs. $\mathrm{H}$. The dotted lines are visual guides...........73

Fig. 5.6 The measured variation of the normalized temperature, $\mathrm{T}_{\mathrm{RH}} \mathrm{vs} . \mathrm{H}^{2 / 3}$. The dotted line and solid lines are for interacting and non-interacting cases respectively .75

Fig. 5.7 Plot of the shift in the peak temperatures $\left[\mathrm{T}_{\mathrm{P}}(0)-\mathrm{T}_{\mathrm{P}}(\mathrm{H})\right]$ against $\mathrm{H}^{2 / 3}$ for the $5 \mathrm{~nm}$ NiO particles. The lines connecting the points are for visual aid. (b) Plot of $\ell n\left[\mathrm{~T}_{\mathrm{P}}(0)-\mathrm{T}_{\mathrm{P}}(\mathrm{H})\right]$ against $\ell n \mathrm{H}$ to check the $\mathrm{H}^{\mathrm{q}}$ variations. ..........76

Fig. 5.8 Isothermal plots of the magnetization $\mathrm{M}$ against applied field $\mathrm{H}$ at

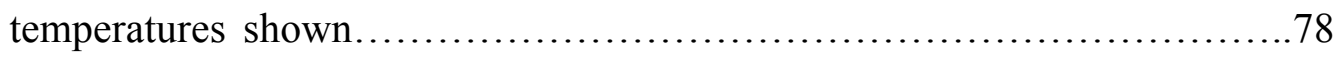


Fig. 5.9 Fitting of the $M$ vs. $H$ data to Eq. (4.16) yielding the plot of $\left(M-\chi_{a} H\right) / M_{0}$ vs. $\mathrm{H} / \mathrm{T}$. The solid line is the Langevin variation with $\mu_{\mathrm{P}}=1240(1000) \mu_{\mathrm{B}}$ for uncoated (coated) NP.

Fig. 5.10 Plot of $\mathrm{M}$ vs. $\mathrm{H}$ measured at $\mathrm{T}=5 \mathrm{~K}$ shows that the coercivity $\mathrm{H}_{\mathrm{c}}$ is smaller for the uncoated particles vs. the coated particles .80

Fig. 5.11 Temperature variation of $\chi^{\prime}$ and $\chi^{\prime \prime}$ in uncoated NiO NP at different frequencies is shown. The lines through the points are for visual aid. .83

Fig. 5.12 Temperature dependence of $\chi^{\prime}$ vs. T for the coated NP. .84

Fig. 5.13 Plot of $\ell n \mathrm{f}$ against $1 / \mathrm{T}_{\mathrm{B}}$ for the coated and uncoated $\mathrm{NiO} \mathrm{NP}$. The solid lines are fits to Eq. (5.8) with parameters given in the plot. The size of error bars for the uncoated sample is within the size of the symbols .85

Fig. 5.14 Plot of $\ell n \mathrm{f}$ against $\mathrm{T}_{\mathrm{B}}$ for the coated and uncoated $\mathrm{NiO} 5 \mathrm{~nm} \mathrm{NP}$. The solid lines are fits to Eq. (5.5) with $\mathrm{T}_{0}=162 \mathrm{~K}$ for the uncoated and $\mathrm{T}_{0}=0 \mathrm{~K}$ for the coated $\mathrm{NiO} \mathrm{NP}$. The magnitudes of $\mathrm{E}_{\mathrm{a}} / \mathrm{k}$ and $\mathrm{f}_{0}$ used in the fit are the same as in Fig. 5.13.

Fig. 6.1 Temperature variation of the magnetic susceptibility $\chi$ of the uncoated NiO-NP measured in $\mathrm{H}=100$ Oe for the FC and ZFC modes. The arrows indicate the location of $\mathrm{T}_{\mathrm{P}}$, the temperatures at which $\chi(\mathrm{ZFC})$ peaks for each case.

Fig. 6.2 Same as Fig. 6.1 except for the OA coated NiO-NP...................92

Fig. 6.3 Variation of $\mathrm{T}_{\mathrm{P}}$ with particle size for the uncoated and coated NiO-NP.

The dotted lines through the points are for a guide to eye.

Fig. 6.4 Plots of measured magnetization $\mathrm{M}$ vs. applied field $\mathrm{H}$ for the uncoated $\mathrm{NiO}-\mathrm{NP}$ at several temperatures above $\mathrm{T}_{\mathrm{P}}$. The lines joining the points are for visual aid.

Fig. 6.5 Same as in Fig. 5.4 except the data are for the OA coated NiO-NP.....97

Fig. 6.6 Plots of $\chi_{\mathrm{a}}$ vs. T show the linear decrease of $\chi_{\mathrm{a}}$ with temperature.........98

Fig. 6.7 Temperature dependence of $\mathrm{M}_{0}$ (Eq. (4.16)) for the uncoated and coated $\mathrm{NiO}-\mathrm{NP}$ for $\mathrm{T}>\mathrm{T}_{\mathrm{P}}$. The solid lines through the data points represent linear variations whereas the dotted lines are extrapolations to $\mathrm{M}_{0} \rightarrow$ zero to estimate the Néel temperature $\mathrm{T}_{\mathrm{N}}$ 
Fig. 6.8 Plots of $\left(\mathrm{M}-\chi_{\mathrm{a}} \mathrm{H}\right) / \mathrm{M}_{0}$ against $\mathrm{H} / \mathrm{T}$ for selected coated and uncoated NiONP. The solid lines are fits to Eq. (4.16) with $\mu_{\mathrm{P}}$ (magnetic moment/particle) values shown on the figures......................................... 100

Fig. 6.9 $\mu_{\mathrm{P}}$ determined at different temperatures by fitting the data of $\mathrm{M}$ vs. $\mathrm{H}$ to Eq. (4.16). For the smaller coated and uncoated NiO-NP, $\mu_{\mathrm{P}}$ so determined is essentially temperature independent.

Fig. 6.10 Plot of $\mu_{P}$ vs. particle size D shows that $\mu_{P}$ increases with increase in D.

Fig. 6.11 Plot of the fraction $f$ of the surface layer spins contributing to $\mu_{P}$ against $D$ for the coated and uncoated NiO-NP. The dashed line is the fitted 1/D variation for the uncoated particles only.

Fig. 6.12 Plots of estimated $\mathrm{T}_{\mathrm{N}}$ (Fig. 6.7) against particle size $\mathrm{D}$ for the coated and uncoated NiO-NP. The dashed line is a theoretical fit with the parameters given in the text................................................... 108

Fig. 6.13 Hysteresis loops for the various particle sizes measured in zero - field cooled (ZFC) and field cooled (FC) cases at $\mathrm{T}=5 \mathrm{~K}$ for the uncoated $\mathrm{NiO}$. 110

Fig. 6.14 Hysteresis loops for the various particle sizes are measured in zero - field cooled (ZFC) and field cooled (FC) cases at $\mathrm{T}=5 \mathrm{~K}$ for coated $\mathrm{NiO}$ samples.

Fig. 6.15 Plot of the variations of loop shift $\mathrm{H}_{\mathrm{E}}$ and coercivity $\mathrm{H}_{\mathrm{C}}$ with particle size under FC and ZFC condition. 


\section{List of Tables}

Table. 2.1 Summary of the values of $J_{1}$ and $J_{2}$ calculated by the Molecular Field theory for AF materials.................................................

Table. 2.2 Difference between $\mathrm{J}_{1}$ and $\mathrm{J}_{2}$, determined by the molecular field theory

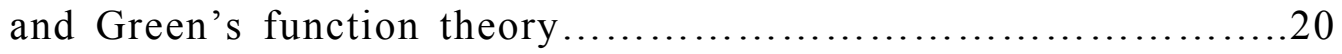

Table. 2.3 Difference of $\mathrm{J}_{1}$ and $\mathrm{J}_{2}$ determined by several methods to compare with the

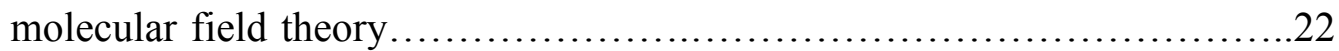

Table. 3.1 The summary of the particle size calculated from TEM analysis comparing the results derived from XRD measurements.............................41

Table. 3.2 Information about each Bragg peak in the x-ray pattern of NiO...........44

Table. 3.3 Summary of the results of the calculation from Fig $3.10 \ldots \ldots \ldots \ldots \ldots \ldots . . . . .46$

Table. 3.4 The Debye-Waller Temperature Factor for all particle sizes..............48

Table. 6.1 Calculated values of the $\mathrm{Ni}^{2+}$ spins for a sphere of diameter $\mathrm{D}: \mathrm{n}_{\mathrm{A}}=\#$ spins on the surface layer; $n_{D}=$ total number of spins; $n_{C}=$ number of spins contributing to $\mu_{\mathrm{P}}$ evaluated from the Langevin fits. Also listed are the ratios

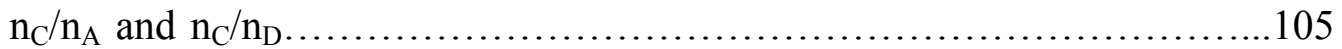

Table. 6.2 The hysteresis loop parameters observed in the ZFC and FC modes are summarized for each particle and here field unit is Oe.....................112 


\section{CHAPTER I}

\section{INTRODUCTION}

\subsection{Magnetic properties of Bulk NiO}

The magnetic properties of bulk $\mathrm{NiO}$ have been studied for several years and are reasonably well understood. $\mathrm{NiO}$ is crystallized in the $\mathrm{NaCl}$ structure and below its Néel temperature $\mathrm{T}_{\mathrm{N}} \approx 523 \mathrm{~K}, \mathrm{Ni}^{2+}$ ions in the ferromagnetic (111) planes are stacked antiferromagnetically along the $<111>$ direction [Shull et al, 2000, Barbier et al, 2004 and Milano et al, 2004]. The antiferromagnetic (AF) order in $\mathrm{NiO}$ originates from the superexchange coupling of $\mathrm{Ni}$ atoms in alternating (111) planes through oxygen $2 \mathrm{p}$ electronic orbitals. $\mathrm{NiO}$ is the $3 \mathrm{~d}$ transition metal (TM) mono-oxide. The orbital moment of the $3 \mathrm{~d}$ TM ion is usually quenched in solids because atomic $3 \mathrm{~d}$ orbitals are greatly deformed in solids by crystal field. However, the spin-orbit interaction in 3d TM monooxides can restore some of the orbital moment of localized $3 \mathrm{~d}$ electrons by reducing crystal field effect. Thus, the experimental magnetic moment / Ni atom $=2.2 \pm 0.2 \mu_{\mathrm{B}}$ is observed with the unquenched orbital moment contribution about $17 \%$ [Neubeck et al, 1999 and Kwon et al, 2000]. The magnitudes of the nearest-neighbor and next-nearest neighbor exchange constants $\mathrm{J}_{1}$ and $\mathrm{J}_{2}$ are calculated as $34 \mathrm{~K}$ and $202 \mathrm{~K}$ respectively using the random-phase-approximation Green's-function theory [Srinivason et al, 1984, Seehra et al, 1988]. The above values are close to the experimental results, from the fit to the experimental spin-wave dispersion curves [Hutchings et al, 1972] and from the Raman scattering data [Dietz et al, 1971].

\subsection{Magnetic properties of NiO Nanoparticles}

In contrast to bulk $\mathrm{NiO}$, the magnetic properties of $\mathrm{NiO}$ in reduced dimensions such as in the nanoparticle (NP) from for sizes D $<30 \mathrm{~nm}$ are not well understood. Since the magnetic nanoparticles have disordered arrangement, particle size distribution, 
random orientation of magnetization, these make their behavior more complex and difficult to understand. Early work [Richardson et al, 1956] investigated the magnetic properties of colloidal $\mathrm{NiO}$ for particle size $\mathrm{D}<200 \mathrm{~nm}$ and particularly, observed peaks in susceptibility measurements for $\mathrm{T}<\mathrm{T}_{\mathrm{N}}$ for crystal sizes 10 to $20 \mathrm{~nm}$. This led Néel to propose the model of superparamagnetism [Néel, 1961] in which large residual moments can occur in AF-NP due to the uncompensated spins especially, on the surface. For the size-range $\mathrm{D} \geq 5 \mathrm{~nm}$, the magnetic properties of NiO-NP have been studied [Makhlouf et al, 1997]. Although their magnetization curves above the blocking temperatures could not be fit into one single curve with a Langevin function, they showed that at $\mathrm{T}=293 \mathrm{~K}$, the magnetic moment per particle $\mu_{\mathrm{P}} \approx 2000 \mu_{\mathrm{B}}$ determined from the Langevin fit is larger by an order of magnitude than the expected value from the two-sublattice model which is applied to bulk NiO. In follow up studies [Kodama et al, 1997 and 1999], numerical modeling of spin configurations yielded 8-, 6-, or 4-sublattice configuration to explain the finite size effects and the observed high coercivities and loop shifts. However they did not address the variation of $\mu_{\mathrm{P}}$ with particle size in their experimental and modeling studies. Also neutron diffraction studies to verify the proposed departures from the two sublattice magnetic ordering have not yet been reported. Recently, Rubinstein et al [2001] have reported on the temperature dependence of the electron magnetic resonance (EMR) spectra of $6 \mathrm{~nm} \mathrm{NiO}$ particles. Similar to the observations reported in ferrrihydrite NP

[Seehra et al. 2001], the resonance line was observed to shift to lower fields with decrease in temperature. However, the observed line shapes were very complex and distorted so that the line-width $\Delta \mathrm{H}$ and the resonance field $\mathrm{H}_{\mathrm{r}}$ could not be measured accurately and hence their temperature variations could not be compared with the predictions for nanoparticle system [Nagata et al. 1992].

\subsection{Motivation for the present work}

Currently, nature of materials in reduced dimensions (thin films, wires, nanoparticles (NP)) has become a very interesting area of study due to their unique magnetic properties as well as their numerous technological applications [Dormann et al. 1992, Kluwer et al. 1994, Speliotis, 1999, and Kodama et al. 1999]. Especially, interest in 
spin ordering at antiferromagnetic (AF) interfaces with ferromagnetic (F) layers [Mocuta et al. 2003, and Ohldag et al. 2001], has strongly increased since the exchange bias at a $\mathrm{F} / \mathrm{AF}$ interface can be applied to lots of fields which rely on exchange bias and spin electronics. Exchange bias at a $\mathrm{F} / \mathrm{AF}$ interface provides an unidirectional magnetic anisotropy resulting a hysteresis loop shift. This property is a key feature in modern magnetic field spin-valve devices. AF $\mathrm{NiO}$ can be a good candidate as $\mathrm{T}_{\mathrm{N}}$ of $\mathrm{NiO}$ is above room temperature. It is of special interest in spin-valve devices which require an $\mathrm{AF} / \mathrm{F}$ (ferromagnet) interface to produce exchange bias at room temperature [Berkowitz et al. 1999 and references therein]. Thus, it is worthy to study the magnetic properties of NiO NP.

This thesis is divided into seven chapters and the contents are as follows. In the second chapter, I discuss magnetic properties of bulk NiO with the Molecular Field theory which is the simplest effective field approximation [Smart, 1966]. Using this theory, I show the three types of antiferromagnetic orderings and ferromagnetic ordering in the face-centered cubic lattice. Curie-Weiss temperature $\theta$ and Néel temperature $T_{N}$ are calculated in terms of exchange constants $J_{1}\left(J_{2}\right)$ for the nearest neighbor (for the next nearest neighbor) and compared with experimental results. In the third chapter, the synthesis procedures of $\mathrm{NiO} \mathrm{NP}$ are specifically introduced. To reduce interparticle interaction, oleic acid (OA) coating is employed. The morphology and structure of the obtained various sized particles are studied through the x-ray diffraction (XRD) and transmission electron microscopy (TEM). Comparison of the sizes determined from the broadening of the $\mathrm{x}$-ray diffraction (XRD) lines and TEM studies shows that TEM studies of NP are important for understanding their structural/magnetic properties. In the fourth chapter, the general properties of superparamagnetism are explained for ferromagnetic and antiferromagnetic systems. In the fifth chapter, one of the important issued in NP magnetism, the effect of interparticle interaction, is focused on to explain the various measured properties with our OA coated and uncoated NiO NP, especially, for the smallest particle with nominal size $5 \mathrm{~nm}$. The coated particles are expected to have much weaker interparticle dipolar interaction vs. the uncoated particles. In the sixth chapter, I focus on the changes in the magnetic properties and morphology of the NiO$\mathrm{NP}$ with increase in the nominal size $\mathrm{D}$ from $5 \mathrm{~nm}$ to about $20 \mathrm{~nm}$. To investigate the 
effect of interparticle interaction, I also provide a comparison between the magnetic properties of the OA coated vs. the uncoated particles. And in the seventh chapter, a summary of the major results of this work is given. 


\section{CHAPTER II}

\section{Molecular Field Theory of Magnetic Ordering}

\subsection{Introduction}

A magnetic ion in a crystal experiences exchange interactions with other magnetic ions. In molecular field theory, these interactions are replaced by an effective field or a molecular field. This discussion of the molecular field theory is based on the information given in the book [Smart, 1996]. Let the two-sublattices consist of "a" and "b" atoms which are ordered antiferromagnetically and the Heisenberg exchange Hamiltonian is given by

$$
\mathrm{H}_{\mathrm{ex}}=-2 \sum_{a \neq b} \mathrm{~J}_{\mathrm{ab}} \mathrm{S}_{\mathrm{a}} \cdot \mathrm{S}_{\mathrm{b}}
$$

where $\mathrm{J}_{\mathrm{ab}}$ is the exchange constant and $\mathrm{S}_{\mathrm{a}}$ and $\mathrm{S}_{\mathrm{b}}$ are the spins on atoms $\mathrm{a}$ and $\mathrm{b}$.

The total fields acting on "a" and "b" atoms are

$$
\begin{aligned}
& \mathrm{H}_{\mathrm{a}}=\mathrm{H}_{0}+\mathrm{H}_{\mathrm{eff}}=\mathrm{H}_{0}+\gamma \mathrm{M}_{\mathrm{b}} \\
& \mathrm{H}_{\mathrm{b}}=\mathrm{H}_{0}+\mathrm{H}_{\mathrm{eff}}=\mathrm{H}_{0}+\gamma \mathrm{M}_{\mathrm{a}}
\end{aligned}
$$

where $\gamma$ is the molecular field coefficient and $\mathrm{H}_{0}$ is applied field. Let $\mathrm{J}_{\mathrm{ab}}$ be non-zero only for the nearest-neighbors which number $\mathrm{z}$.

Then

$$
<\mathrm{H}_{\mathrm{ex}}>=-2 \mathrm{~J}_{\mathrm{ab}}<\mathrm{S}_{\mathrm{a}}>\cdot \sum<\mathrm{S}_{\mathrm{b}}>
$$

The expectation values of $\mathrm{H}_{\mathrm{ex}}$ can be written as

$$
<\mathrm{H}_{\mathrm{ex}}>=-\mathrm{g} \mu_{\mathrm{B}} \mathrm{H}_{\mathrm{eff}} \cdot<\mathrm{S}_{\mathrm{a}}>
$$

By substituting Eq. (2.4) into (2.5), we have

$$
\mathrm{H}_{\text {eff }}=2 \mathrm{z} \mathrm{J} \mathrm{Ja}_{\mathrm{ab}}<\mathrm{S}_{\mathrm{b}}>/ \mathrm{g} \mu_{\mathrm{B}}=\gamma \mathrm{M}=\gamma \mathrm{g} \mu_{\mathrm{B}} \mathrm{N}_{\mathrm{a}}<\mathrm{S}_{\mathrm{b}}>
$$

where $\mathrm{N}_{\mathrm{a}}$ is the number of atom in "a" sublattice, $\mu_{\mathrm{B}}$ is the Bohr magneton and $\mathrm{g}$ is the Landé g-factor.

So, we have 


$$
\gamma=2 \mathrm{~J}_{\mathrm{ab}} \mathrm{z} / \mathrm{N}_{\mathrm{a}} \mathrm{g}^{2} \mu_{\mathrm{B}}^{2} \text {. }
$$

Eq. (2.7) shows that $\gamma$ is proportional to the exchange constant $\mathrm{J}_{\mathrm{ab}}$. The transition metal oxides, $\mathrm{MnO}, \mathrm{NiO}, \mathrm{CoO}$, and $\mathrm{FeO}$ crystallize in the $\mathrm{NaCl}$ (FCC) structure. Since there are four (4) magnetic ions (e.g. $\mathrm{Ni}^{2+}$ ) per unit cell, the two sublattice model is inadequate. In comparison, the SC lattice has only one atom/cell and BCC lattice has two atoms per unit cell. When a material orders antiferromagnetically (AF) below its Néel temperature, $\mathrm{T}_{\mathrm{N}}$, the size of the magnetic unit cell becomes double that of the chemical unit cell because of AF ordering. Therefore, the magnetic unit cell of the corresponding $\mathrm{SC}, \mathrm{BCC}$, and $\mathrm{FCC}$ chemical unit cells have two, four, and eight magnetic ions respectively. Thus, in describing ordering in SC, BCC, and FCC cells, we need to consider 2, 4, and 8 sublattices respectively. For discussing ordering in $\mathrm{NiO}$, the $\mathrm{Ni}^{2+}$ ions are divided into eight sublattices as shown in Fig.2.1.

\subsection{The secular equation}

We begin with the general equation of the exchange Heisenberg Hamiltonian

$$
\mathrm{H}=-2 \sum_{i \neq j} \mathrm{~J}_{\mathrm{ij}} \mathrm{S}_{\mathrm{i}} \cdot \mathrm{S}_{\mathrm{j}}
$$

where $\mathrm{J}_{\mathrm{ij}}$ is the exchange interaction between an $\mathrm{i}$ atom and one of its $\mathrm{j}$ neighbors.

The total field acting on one atom of the $i^{\text {th }}$ sublattice is

$$
\mathrm{H}_{\mathrm{i}}=\mathrm{H}_{0}+\sum_{\substack{j=1 \\ i \neq j}}^{n} \gamma_{\mathrm{ij}} \mathrm{M}_{\mathrm{j}}
$$

where $\gamma_{\mathrm{ij}}$ is the molecular field coefficient, $\mathrm{M}_{\mathrm{j}}$ is the magnetization of the $\mathrm{j}^{\mathrm{t}}$ sublattice and $\mathrm{n}$ is the number of sublattices.

Following the Eq. (2.7), we have

$$
\begin{array}{ll}
\gamma_{i j}=0 & \text { for } i=j \\
\gamma_{i j}=n\left(2 J_{i j} z_{i j}\right) / N g^{2} \mu_{B}^{2} & \text { for } i \neq j
\end{array}
$$

where $\mathrm{z}_{\mathrm{ij}}$ is the number of $\mathrm{j}$ neighbors of an $\mathrm{i}$ atom and $\mathrm{N}$ is the total number of atoms. 
Eq. (2.10) and Eq. (2.11) state that atoms interact with atoms on the other sublattices only. For $\mathrm{NiO}$, a given atom has 12 nearest neighbors at a distance of a/ $\sqrt{2}$ on six different lattices and six next-nearest neighbors on the cubic edges at a distance of a.

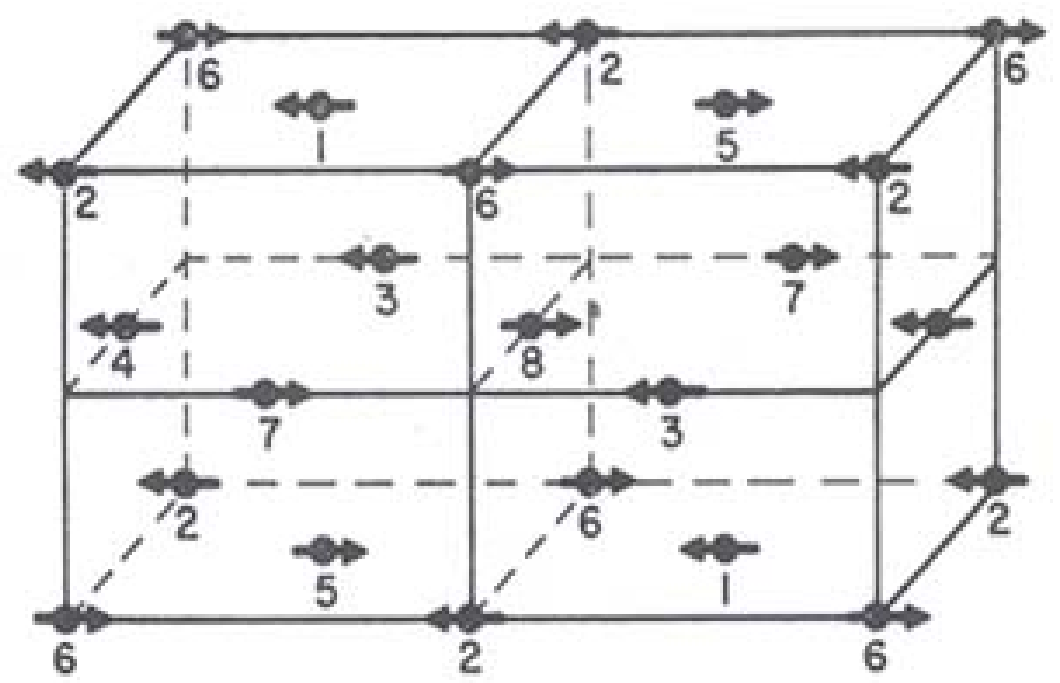

Fig. 2.1 The face-centered cubic lattice divided into eight sublattices. 
Thus, from the Fig.2.1, let $\gamma_{12}=\gamma_{13}=\gamma_{14}=\gamma_{16}=\gamma_{17}=\gamma_{18}$ be $\gamma_{1}$ for the nearest neighbors (nm) and $\gamma_{15}$ be $\gamma_{2}$ for the next-nearest neighbors (nnm). For each $\mathrm{H}$,

$$
\begin{aligned}
& \mathrm{H}_{1}=\mathrm{H}_{0}+8 / 6 \gamma_{1} \mathrm{M}_{2}+\ldots \ldots+8 \gamma_{2} \mathrm{M}_{5}+\ldots . .+8 / 6 \gamma_{1} \mathrm{M}_{8} \\
& \mathrm{H}_{2}=\mathrm{H}_{0}+8 / 6 \gamma_{1} \mathrm{M}_{1}+\ldots \ldots+8 \gamma_{2} \mathrm{M}_{6}+\ldots . .+8 / 6 \gamma_{1} \mathrm{M}_{8} \\
& \ldots \ldots \ldots \ldots \\
& \mathrm{H}_{8}=\mathrm{H}_{0}+8 / 6 \gamma_{1} \mathrm{M}_{1}+\ldots \ldots+8 \gamma_{2} \mathrm{M}_{4}+\ldots . .+8 / 6 \gamma_{1} \mathrm{M}_{7}
\end{aligned}
$$

The relation of $\mathrm{M}$ and $\mathrm{H}$ is represented by the Curie law,

$$
\begin{aligned}
\mathrm{M}_{\mathrm{i}} & =\mathrm{C} / \mathrm{nT} \mathrm{H}_{\mathrm{i}} \\
& =\mathrm{C} / \mathrm{nT}\left(\mathrm{H}_{0}+\sum \gamma_{\mathrm{ij}} \mathrm{M}_{\mathrm{j}}\right)
\end{aligned}
$$

where the Curie constant, $\mathrm{C}=\mathrm{N} / 8 \mathrm{~g}^{2} \mu_{\mathrm{B}}^{2} \mathrm{~S}(\mathrm{~S}+1) / 3 \mathrm{k}_{\mathrm{B}}$.

So, $\mathrm{H}_{1}, \mathrm{H}_{2}, \ldots$, and $\mathrm{H}_{8}$ in Eq. (2.12) can be replaced by $8 \mathrm{~T} / \mathrm{C} \mathrm{M}_{1}, 8 \mathrm{~T} / \mathrm{C} \mathrm{M}_{2}, \ldots$, and $8 \mathrm{~T} / \mathrm{C} \mathrm{M}_{8}$. We can rewrite Eq. (2.12) as

$$
\begin{aligned}
8 \mathrm{~T} / \mathrm{C} \mathrm{M}_{1}-8 / 6 \gamma_{1} \mathrm{M}_{2}-\ldots-8 \gamma_{2} \mathrm{M}_{5}-\ldots-8 / 6 \gamma_{1} \mathrm{M}_{8} & =\mathrm{H}_{0} \\
-8 / 6 \gamma_{1} \mathrm{M}_{1}+8 \mathrm{~T} / \mathrm{C} \mathrm{M}_{2}-\ldots-8 \gamma_{2} \mathrm{M}_{6}-\ldots-8 / 6 \gamma_{1} \mathrm{M}_{8} & =\mathrm{H}_{0} \\
\quad \ldots \ldots \ldots \ldots . . & \\
-8 / 6 \gamma_{1} \mathrm{M}_{1}-8 / 6 \gamma_{1} \mathrm{M}_{2}-\ldots . & 8 \gamma_{2} \mathrm{M}_{4}-\ldots .+8 \mathrm{~T} / \mathrm{C} \mathrm{M}_{8}=\mathrm{H}_{0}
\end{aligned}
$$

Eq. (2.14) can be represented by the matrix with the applied field $\mathrm{H}_{0}=0$ and the determinant of the secular equation is(with $\mathrm{a}_{0}=8 \mathrm{~T} / \mathrm{C}, \mathrm{a}_{1}=-4 / 3 \gamma_{1}, \mathrm{a}_{2}=-8 \gamma_{2}$ ),

$$
\left|\begin{array}{cccccc}
\mathrm{a}_{0} & \mathrm{a}_{1} & \ldots & \mathrm{a}_{2} & \ldots \ldots & \mathrm{a}_{1} \\
\mathrm{a}_{1} & \mathrm{a}_{0} & \ldots & \mathrm{a}_{2} & \ldots . & \mathrm{a}_{1} \\
: & : & \ldots & : & \ldots . \\
\mathrm{a}_{2} & \mathrm{a}_{1} & \ldots & \mathrm{a}_{0} & \ldots & \mathrm{a}_{1} \\
: & : & \ldots & : & \ldots . \\
\mathrm{a}_{1} & \mathrm{a}_{1} & \ldots & \mathrm{a}_{2} & \ldots \ldots \\
& & & & \ldots & \mathrm{a}_{0}
\end{array}\right|=0
$$


The eight solutions of Eq. (2.15) yield the transition temperatures. To solve this Eq. (2.15), the standard procedure of subtracting rows and columns is employed (without affecting the solutions), leading to only three independent solutions due to degeneracy. The first factor leads to quadruple degeneracy, $\left(a_{0}-a_{2}\right)^{4}=0$ or $a_{0}=a_{2}$.

These yield

$$
\mathrm{T}_{\mathrm{N} 2}=-\mathrm{C} \gamma_{2}
$$

In the remaining determinant, add row 1 to row 5 . This changes the determinant $8 \times 8$ to $7 \times 7$. Repeat this step until the determinant becomes $4 \times 4$. Thus we finally get a $3 \times 3$ matrix,

$$
-A\left|\begin{array}{rrc}
-\mathrm{A} & -\mathrm{A} & \mathrm{A}+4 \mathrm{a}_{1} \\
\mathrm{~A} & 0 & 2 \mathrm{a}_{1} \\
0 & \mathrm{~A} & 2 \mathrm{a}_{1}
\end{array}\right| \quad=0
$$

where $A \equiv\left(a_{2}+a_{0}-2 a_{1}\right)$

So, we have the results of Eq. (2.17) as

$$
\begin{array}{lll} 
& \mathrm{A}^{3}=0, & \text { triple roots } \\
\text { And } \quad \mathrm{A}=-8 \mathrm{a}_{1}, & \text { single root }
\end{array}
$$

From Eq. (2.18) and Eq. (2.19) respectively

$$
\begin{aligned}
& \mathrm{T}_{\mathrm{N}}=\mathrm{C}\left(-1 / 3 \gamma_{1}+\gamma_{2}\right) \\
& \mathrm{T}_{\mathrm{N}}=\mathrm{C}\left(\gamma_{1}+\gamma_{2}\right)
\end{aligned}
$$

The eigen vectors for each transition temperature can be obtained by substituting each solution of the matrix into original matrix. For the first case of $\mathrm{a}_{0}=\mathrm{a}_{2}$, the result is

$$
a_{0}\left(M_{1}+M_{5}\right)+a_{1}\left(M_{2}+M_{3}+\ldots . .+M_{8}\right)=0
$$

This means

$$
\mathrm{M}_{1}=-\mathrm{M}_{5}
$$


Similarly

$$
M_{2}=-M_{6}, M_{3}=-M_{7}, M_{4}=-M_{8}
$$

For the solution, $\mathrm{a}_{2}+\mathrm{a}_{0}-2 \mathrm{a}_{1}=0$,

$$
\begin{aligned}
& a_{0}\left(M_{1}+M_{2}+M_{3}+M_{4}\right)-a_{1}\left(M_{5}+M_{6}+M_{7}+M_{8}\right) \\
& \quad+a_{1}\left\{3\left(M_{1}+M_{2}+M_{3}+M_{4}\right)+5\left(M_{5}+M_{6}+M_{7}+M_{8}\right)\right\}=0
\end{aligned}
$$

The eigen vectors of this equation are

$$
\begin{aligned}
& M_{1}=M_{5}, M_{2}=M_{6}, M_{3}=M_{7}, M_{4}=M_{8} \\
& \text { and } M_{1}+M_{2}+M_{3}+M_{4}=M_{5}+M_{6}+M_{7}+M_{8}=0
\end{aligned}
$$

And for the last case, $\mathrm{a}_{2}+\mathrm{a}_{0}+6 \mathrm{a}_{1}=0$,

$$
a_{0}\left(M_{1}-M_{5}\right)+a_{1}\left(M_{2}+M_{3}+M_{4}-6 M_{5}+M_{6}+M_{7}+M_{8}\right)=0
$$

So,

$$
\mathrm{M}_{1}=\mathrm{M}_{2}=\mathrm{M}_{3}=\mathrm{M}_{4}=\mathrm{M}_{5}=\mathrm{M}_{6}=\mathrm{M}_{7}=\mathrm{M}_{8}
$$

In summary, the solutions are

First case, $\quad \mathrm{T}_{\mathrm{N} 2}=-\mathrm{C} \gamma_{2}$, where $\mathrm{J}_{2}<0$

$$
\mathrm{M}_{1}=-\mathrm{M}_{5}, \mathrm{M}_{2}=-\mathrm{M}_{6}, \mathrm{M}_{3}=-\mathrm{M}_{7} \text {, and } \mathrm{M}_{4}=-\mathrm{M}_{8}
$$

This is what is observed in $\mathrm{NiO}$ and represents the AF ordering of type II.

Second case, $\quad \mathrm{T}_{\mathrm{N} 1}=\mathrm{C}\left(-1 / 3 \gamma_{1}+\gamma_{2}\right)$, where $\mathrm{J}_{1}<0$ and $\mathrm{J}_{2}>0$

$$
\begin{gathered}
\mathrm{M}_{1}=\mathrm{M}_{5}, \mathrm{M}_{2}=\mathrm{M}_{6}, \mathrm{M}_{3}=\mathrm{M}_{7}, \mathrm{M}_{4}=\mathrm{M}_{8} \\
\text { and } \mathrm{M}_{1}+\mathrm{M}_{2}+\mathrm{M}_{3}+\mathrm{M}_{4}=\mathrm{M}_{5}+\mathrm{M}_{6}+\mathrm{M}_{7}+\mathrm{M}_{8}=0
\end{gathered}
$$

This one shows the AF ordering for type I

And the last case, $\quad \mathrm{T}_{\mathrm{C}}=\mathrm{C}\left(\gamma_{1}+\gamma_{2}\right)$, where $\mathrm{J}_{1}>0$ and $\mathrm{J}_{2}>0$

$$
\mathrm{M}_{1}=\mathrm{M}_{2}=\mathrm{M}_{3}=\mathrm{M}_{4}=\mathrm{M}_{5}=\mathrm{M}_{6}=\mathrm{M}_{7}=\mathrm{M}_{8}
$$

This case is the ferromagnetic state. 


\subsection{Three types of ordering and equations for $T_{N}$ and $\theta$}

Eq. (2.27) describes the antiferromagnetic ordering of magnetic atoms. Atoms are ordered ferromagnetically in $\mathrm{x}-\mathrm{y}$ plane and the direction of spins is changed alternately as shown in Fig. 2.2 (a) choosing $\mathrm{M}_{1}=\mathrm{M}_{2}=-\mathrm{M}_{3}=-\mathrm{M}_{4}$. This arrangement can also occur in $\mathrm{y}-\mathrm{z}$ plane and $\mathrm{x}-\mathrm{z}$ plane. The number of nearest neighbor pairs ordered antiferromagnetically is the largest among the other orderings. So this is called the antiferromagnetic ordering of type I. The feature of ordering in Eq. (2.26) also represents antiferromagnetic ordering. The atoms within planes perpendicular to a particular cube diagonal are ordered ferromagnetically and the directions of these atoms are antiparallel with adjacent planes as shown in Fig.2.2 (b). This is called antiferromagnetic ordering of type II. There is another type of antiferromagnetic ordering called antiferromagnetic ordering type III. This ordering is similar to type I with the only difference being the change in the direction of one spin at the cube edge followed by the same sublattice. This is shown in Fig. 2.2 (c). And in the last case shown in Eq. (2.28), $\mathrm{M}_{1}, \mathrm{M}_{2}, \ldots$, and $\mathrm{M}_{8}$, all have same magnitude and direction, leading to the ferromagnetic state.

The Curie-Weiss temperature $\theta$ and the Néel temperature $\mathrm{T}_{\mathrm{N}}$ for the $\mathrm{NiO}$ system can be represented in terms of exchange constants $J_{1}$ and $J_{2}$. Eq. (2.14) can be written as

$$
\begin{aligned}
\mathrm{M}_{1} & =\mathrm{C} / 8 \mathrm{~T}\left\{\mathrm{H}_{0}+4 / 3 \gamma_{1}\left(\mathrm{M}_{2}+\ldots+\mathrm{M}_{8}\right)+8 \gamma_{2} \mathrm{M}_{5}\right\} \\
\mathrm{M}_{2} & =\mathrm{C} / 8 \mathrm{~T}\left\{\mathrm{H}_{0}+4 / 3 \gamma_{1}\left(\mathrm{M}_{1}+\ldots+\mathrm{M}_{8}\right)+8 \gamma_{2} \mathrm{M}_{6}\right\} \\
& \ldots \ldots \ldots \ldots \\
\mathrm{M}_{8} & =\mathrm{C} / 8 \mathrm{~T}\left\{\mathrm{H}_{0}+4 / 3 \gamma_{1}\left(\mathrm{M}_{1}+\ldots+\mathrm{M}_{7}\right)+8 \gamma_{2} \mathrm{M}_{4}\right\}
\end{aligned}
$$

In the paramagnetic region,

$$
\begin{aligned}
\mathrm{M} & =\sum \mathrm{M}_{\mathrm{i}} \\
& =\mathrm{C} / 8 \mathrm{~T}\left\{8 \mathrm{H}_{0}+4 / 3 \gamma_{1} 6\left(\mathrm{M}_{1}+\ldots+\mathrm{M}_{8}\right)+8 \gamma_{2}\left(\mathrm{M}_{1}+\ldots+\mathrm{M}_{8}\right)\right\} \\
& =\mathrm{C} / \mathrm{T}\left\{\mathrm{H}_{0}+\left(\gamma_{1}+\gamma_{2}\right) \mathrm{M}\right\} \\
\mathrm{M} & =\mathrm{C} / \mathrm{T} \mathrm{H}_{0} /\left\{1-\mathrm{C} / \mathrm{T}\left(\gamma_{1}+\gamma_{2}\right)\right\}
\end{aligned}
$$

And

$$
\chi=\partial \mathrm{M} / \partial \mathrm{H}_{0}=\mathrm{C} /\left\{\mathrm{T}-\mathrm{C}\left(\gamma_{1}+\gamma_{2}\right)\right\}=\mathrm{C} /\{\mathrm{T}+|\theta|\}
$$

where the paramagnetic transition temperature, $\theta=\left|-C\left(\gamma_{1}+\gamma_{2}\right)\right|$. 


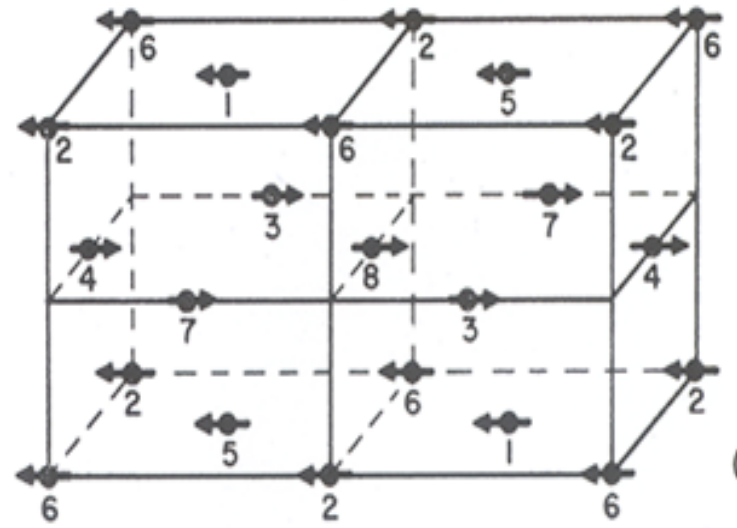

(a)

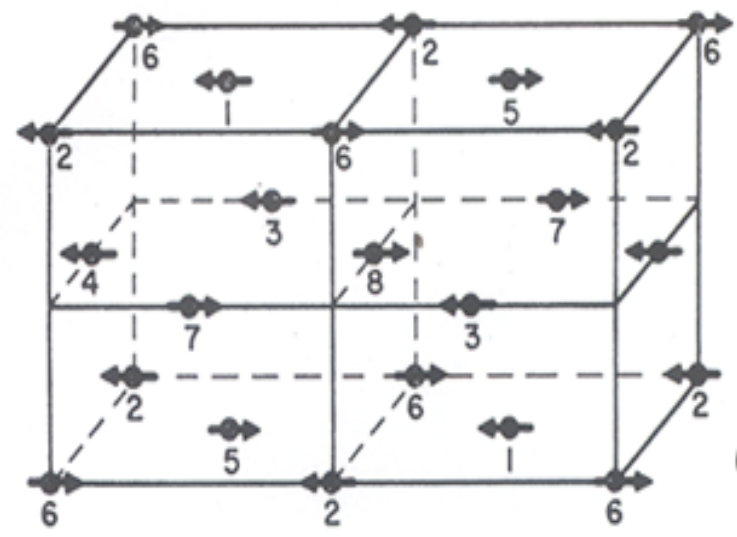

(b)

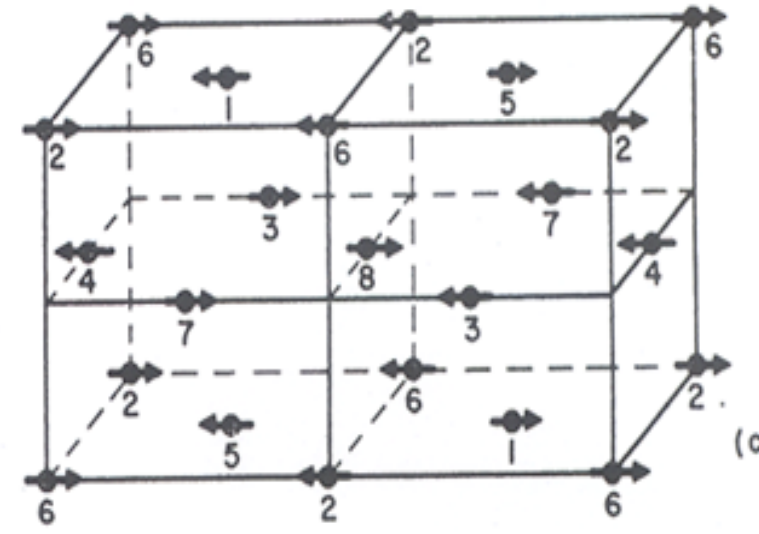

(c)

Fig. 2.2 Antiferromagnetic types in FCC lattice. 
This FCC system has 12 nearest neighbors and 6 next nearest neighbors. From Eq. (2.11),

$$
\text { and } \quad \begin{aligned}
\gamma_{1} & =(2 \times 12) \mathrm{J}_{1} /\left(\mathrm{N} / 8 \mathrm{~g}^{2} \mu_{\mathrm{B}}{ }^{2}\right), \\
\gamma_{2} & =(2 \times 6) \mathrm{J}_{2} /\left(\mathrm{N} / 8 \mathrm{~g}^{2} \mu_{\mathrm{B}}{ }^{2}\right)
\end{aligned}
$$

Thus, the transition temperature $\theta$ has the form,

$$
\theta=2 \mathrm{~S}(\mathrm{~S}+1)\left(12 \mathrm{~J}_{1}+6 \mathrm{~J}_{2}\right) / 3 \mathrm{k}_{\mathrm{B}}
$$

Similarly, the transition temperatures for each ordering type, determined for $\mathrm{H}_{0}=0$, are also given by $\mathrm{J}_{1}$ and $\mathrm{J}_{2}$.

In Eq. (2.28),

$$
\begin{aligned}
\mathrm{T}_{\mathrm{C}} & =\mathrm{C}\left(\gamma_{1}+\gamma_{2}\right) \\
& =2 \mathrm{~S}(\mathrm{~S}+1)\left(12 \mathrm{~J}_{1}+6 \mathrm{~J}_{2}\right) / 3 \mathrm{k}_{\mathrm{B}}
\end{aligned}
$$

In Eq. (2.27),

$$
\begin{aligned}
\mathrm{T}_{\mathrm{N} 1} & =\mathrm{C}\left(-1 / 3 \gamma_{1}+\gamma_{2}\right) \\
& =2 \mathrm{~S}(\mathrm{~S}+1)\left(-4 \mathrm{~J}_{1}+6 \mathrm{~J}_{2}\right) / 3 \mathrm{k}_{\mathrm{B}}
\end{aligned}
$$

And in Eq. (2.26),

$$
\begin{aligned}
\mathrm{T}_{\mathrm{N} 2} & =-\mathrm{C} \gamma_{2} \\
& =-2 \mathrm{~S}(\mathrm{~S}+1)\left(6 \mathrm{~J}_{2}\right) / 3 \mathrm{k}_{\mathrm{B}}
\end{aligned}
$$

For the antiferromagnetic ordering of type III, P.W. Anderson [1950] proposed the magnetic structure given by the transition temperature $\mathrm{T}_{\mathrm{N} 3}$.

$$
\begin{aligned}
\mathrm{T}_{\mathrm{N} 3} & =\mathrm{C} / 3\left(-\gamma_{1}+\gamma_{2}\right) \\
& =2 \mathrm{~S}(\mathrm{~S}+1)\left(-4 \mathrm{~J}_{1}+2 \mathrm{~J}_{2}\right) / 3 \mathrm{k}_{\mathrm{B}}
\end{aligned}
$$

The results for the transition temperatures show that, in ferromagnetic ordering, the real transition temperature, $T_{C}$, and the paramagnetic transition temperature, $\theta$, are the same in Eq. (2.34) and Eq. (2.35). But the transition temperatures, $T_{N}$, for the antiferromagnetic ordering are different depending on the magnetic ordering. 


\subsection{The $J_{1}-J_{2}$ diagram for $F C C$ systems}

In a paper in 1988, Seehra and Giebultowitz investigated the relationship between the magnetic structures of FCC systems and the exchange constants $J_{1}$ and $J_{2}$. Using the values of $J_{1}$ and $J_{2}$ which are known accurately from experiments, they drew the phase diagram shown in Fig. 2.3. The exchange constants $J_{1}$ and $J_{2}$ are dominant in systems where magnetic ions occupy the FCC lattice sites and there exists four types of orders in magnetic structure for the values of $J_{1}$ and $J_{2}$ except where $J_{1}>0$ and $J_{2}=0$. The three antiferromagnetic orderings and ferromagnetic ordering are possible as calculated in previous section.

In Fig. 2.3, phase boundaries are shown determined by making the differences in the transition temperatures in Eq. (2.35), Eq. (2.36), Eq. (2.37), and Eq. (2.38) to equal zero. For Eq. (2.35) and Eq. (2.36), the difference is

$$
\mathrm{T}_{\mathrm{C}}-\mathrm{T}_{\mathrm{N} 1}=2 \mathrm{~S}(\mathrm{~S}+1)\left(16 \mathrm{~J}_{1}\right) / 3 \mathrm{k}_{\mathrm{B}}
$$

Thus, the boundary of these regions, $\mathrm{J}_{1}=0$ is obtained by allowing Eq. (2.39) to equal zero.

Similarly, for Eq. (2.35) and Eq. (2.37), $\quad \mathrm{J}_{1}=-\mathrm{J}_{2}$

for Eq. (2.37) and Eq. (2.38), $\quad \mathrm{J}_{1}=2 \mathrm{~J}_{2}$

for Eq. (2.36) and Eq. (2.38), $\quad \mathrm{J}_{2}=0$

That is,

$$
\begin{array}{ll}
\mathrm{J}_{1}=0 & \text { between AFI and F } \\
\mathrm{J}_{1}=-\mathrm{J}_{2} & \text { between F and AFII } \\
\mathrm{J}_{1}=2 \mathrm{~J}_{2} & \text { between AFII and AFIII } \\
\mathrm{J}_{2}=0 & \text { between AFIII and AFI }
\end{array}
$$

These authors suggested that the closer a magnetic structure gets to the boundary, the more unstable it becomes. For instance, EuSe is located close to the boundary and experimentally shows that magnetic ordering is changed from AFII to F even in small difference of temperature. And the materials in AFII are quite stable since they all are further away from the boundaries. Thus, if $\mathrm{J}_{1}$ and $\mathrm{J}_{2}$ are known accurately among the FCC systems, transition can be predicted. 


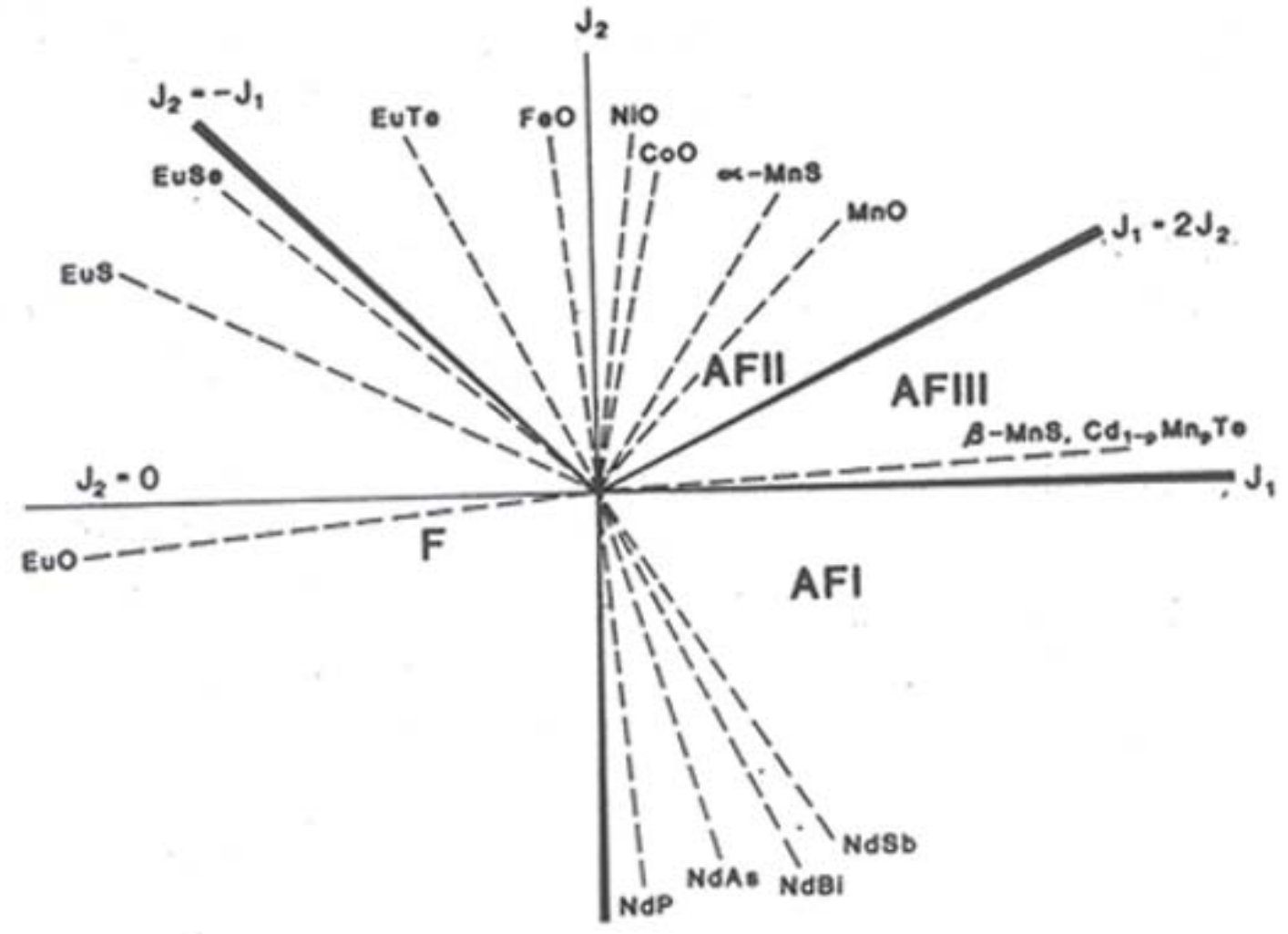

Fig.2.3 Phase diagram of FCC systems with $\mathrm{J}_{1}$ and $\mathrm{J}_{2}$ [Seehra et al, 1988]. 


\section{$2.5 J_{1}$ and $J_{2}$ for the FCC system}

In section 2.3, equations for $\mathrm{T}_{\mathrm{N}}$ and $\theta$ are derived using the molecular field theory. Many experiments show the values of $\mathrm{T}_{\mathrm{N}}$ and $\theta$ for particular materials [Smart, 1996] and $J_{1}$ and $J_{2}$ can be calculated by substituting these values into the equations for $T_{N}$ and $\theta$. Table 2.1 shows the calculated values of $\mathrm{J}_{1}$ and $\mathrm{J}_{2}$ for the FCC systems.

For $\mathrm{NiO}$ with $\mathrm{S}=1$, Eq. (2.34) and Eq. (2.37) are

$$
\begin{aligned}
& \theta=2 \mathrm{~S}(\mathrm{~S}+1)\left(12 \mathrm{~J}_{1}+6 \mathrm{~J}_{2}\right) / 3 \mathrm{k}_{\mathrm{B}}=-1310 \mathrm{~K} \\
& \mathrm{~T}_{\mathrm{N} 2}=-2 \mathrm{~S}(\mathrm{~S}+1)\left(6 \mathrm{~J}_{2}\right) / 3 \mathrm{k}_{\mathrm{B}}=523 \mathrm{~K}
\end{aligned}
$$

Thus, we obtain $\mathrm{J}_{1} / \mathrm{k}_{\mathrm{B}}=-81.9$ and $\mathrm{J}_{2} / \mathrm{k}_{\mathrm{B}}=-65.4$ for $\mathrm{NiO}$ from Eq. (2.41). $\mathrm{J}_{1}$ and $\mathrm{J}_{2}$ for the other compounds are calculated in a similar way for each magnetic ordering type.

The above calculated values of $\mathrm{J}_{1}$ and $\mathrm{J}_{2}$ for $\mathrm{NiO}$ are quite different from the experimental values obtained in neutron scattering experiments. Experimental value estimates that $J_{1}$ is approximately zero and $J_{2}$ has a value much bigger than $J_{1}$. It shows that the molecular field theory does not always agree with the actual exchange values since this theory neglects higher order interactions. In the paper [Srinivasan et al, 1983], the authors solved this difficulty using Green's function analysis which is discussed in the next section. 


\begin{tabular}{|c|c|c|c|c|c|}
\hline Compound & $\begin{array}{l}\text { Type of } \\
\text { ordering }\end{array}$ & $\mathrm{T}_{\mathrm{N}}\left({ }^{\circ} \mathrm{K}\right)$ & $\theta\left({ }^{\circ} \mathrm{K}\right)$ & $-\mathrm{J}_{1} / \mathrm{k}_{\mathrm{B}}$ & $-\mathrm{J}_{2} / \mathrm{k}_{\mathrm{B}}$ \\
\hline $\mathrm{MnO}$ & AFII & 116 & -610 & 7.1 & 3.3 \\
\hline $\mathrm{FeO}$ & AFII & 198 & -570 & 7.8 & 8.3 \\
\hline $\mathrm{CoO}$ & AFII & 292 & -330 & 1.3 & 19.5 \\
\hline $\mathrm{NiO}$ & AFII & 523 & -1310 & 81.9 & 65.4 \\
\hline$\alpha-\mathrm{MnS}$ & AFII & 154 & -465 & 4.4 & 4.4 \\
\hline $\mathrm{MnS}_{2}$ & AFIII & 60 & -592 & 5.5 & 5.9 \\
\hline $\mathrm{MnTe}_{2}$ & AFI & 100 & -528 & 6.7 & 1.6 \\
\hline
\end{tabular}

Table. 2.1. The summary of the values of $J_{1}$ and $J_{2}$ calculated by the Molecular Field theory for AF materials. 


\subsection{Results of the Green's Function Theory for $\mathbf{J}_{\mathbf{1}}$ and $\mathbf{J}_{\mathbf{2}}$}

There is another method to evaluate $\mathrm{J}_{1}$ and $\mathrm{J}_{2}$, the nearest neighbor and nextnearest neighbor exchange constants, in the molecular field theory. In the previous section, $\mathrm{J}_{1}$ and $\mathrm{J}_{2}$ are determined from the transition temperature $\mathrm{T}_{\mathrm{N}}$ and paramagnetic transition temperature $\theta$. However, for some materials, it is difficult to measure exact $\theta$ experimentally. Thus, susceptibility $\chi$ is used instead of $\theta$. In Eq. (2.32), susceptibility $\chi$ is $\chi(\mathrm{T})=\mathrm{C} /\left\{\mathrm{T}-\mathrm{C}\left(\gamma_{1}+\gamma_{2}\right)\right\}$, where $\mathrm{C}=(\mathrm{N} / 8) \mathrm{g}^{2} \mu_{\mathrm{B}}^{2} \mathrm{~S}(\mathrm{~S}+1) / 3 \mathrm{k}_{\mathrm{B}}$. For the case of antiferromagnetic type II, the transition temperature $T_{N}$ is

$$
\mathrm{T}_{\mathrm{N}}=-4 \mathrm{~S}(\mathrm{~S}+1)\left(\mathrm{J}_{2}\right) / \mathrm{k}_{\mathrm{B}}
$$

And at this transition $\mathrm{T}_{\mathrm{N}}$, susceptibility $\chi$ is

$$
\chi\left(\mathrm{T}_{\mathrm{N}}\right)=-\mathrm{Ng}^{2} \mu_{\mathrm{B}}^{2} / 16\left\{12\left(\mathrm{~J}_{1}+\mathrm{J}_{2}\right)\right\} .
$$

In the paper [Srinivasan et al, 1984], the authors calculate the values of $\mathrm{J}_{1}$ and $\mathrm{J}_{2}$ for $\mathrm{NiO}$, with the values of $\chi\left(\mathrm{T}_{\mathrm{N}}\right)=8.8 \times 10^{-6} \mathrm{~cm}^{3} / \mathrm{g}, \mathrm{S}=1, \mathrm{~g}=2.23$ and $\mathrm{T}_{\mathrm{N}}=524 \mathrm{~K}$. From the Eq. (2.42) and Eq. (2.43), they came up with

$$
\mathrm{J}_{1} / \mathrm{k}_{\mathrm{B}}=-51.5 \mathrm{~K} \text { and } \mathrm{J}_{2} / \mathrm{k}_{\mathrm{B}}=-65.5 \mathrm{~K}
$$

It is well known that the molecular field theory is not always a good approximation. Thus, the authors attempt to estimate $\mathrm{J}_{1}$ and $\mathrm{J}_{2}$ using the random-phase-approximation Green's function theory. Bartel and Morosin [1971] calculated the relation of $\mathrm{k}_{\mathrm{B}} \mathrm{T}_{\mathrm{N}} / \mathrm{J}_{2}$ versus $\mathrm{J}_{1} / \mathrm{J}_{2}$ for $\mathrm{S}=1$. This figure is shown in Fig. 2.4 which used two times larger values of $\mathrm{J}_{1}$ and $\mathrm{J}_{2}$ as the authors start from Hamiltonian $\mathrm{H}=-\sum \mathrm{J}_{\mathrm{ij}} \mathrm{S}_{\mathrm{i}} \cdot \mathrm{S}_{\mathrm{j}}$ to obtain $\mathrm{J}_{1}$ and $\mathrm{J}_{2}$. First they assume $\mathrm{J}_{1}=0$ which leads $\mathrm{k}_{\mathrm{B}} \mathrm{T}_{\mathrm{N}} / \mathrm{J}_{2}=2.67$ and $\mathrm{J}_{2} / \mathrm{k}_{\mathrm{B}}$ is calculated to $-196 \mathrm{~K}$ yielding to half reduced $\mathrm{J}_{2} / \mathrm{k}_{\mathrm{B}}=-98 \mathrm{~K}$ in our notation. From Eq. $(2.43),\left(\mathrm{J}_{1}+\mathrm{J}_{2}\right)=-117 \mathrm{~K}$ for $\chi$ $\left(\mathrm{T}_{\mathrm{N} 2}\right)=8.8 \times 10^{-6} \mathrm{~cm}^{3} / \mathrm{g}$ and $\mathrm{J}_{2} / \mathrm{k}_{\mathrm{B}}=-98 \mathrm{~K}$ yield $\mathrm{J}_{1} / \mathrm{k}_{\mathrm{B}}=-19 \mathrm{~K}$. This in turn represents the ratio, $\mathrm{J}_{1} / \mathrm{J}_{2}=0.19$, yielding $\mathrm{k}_{\mathrm{B}} \mathrm{T}_{\mathrm{N}} / \mathrm{J}_{2}=2.626$. Thus, again $\mathrm{J}_{2} / \mathrm{k}_{\mathrm{B}}=-99.6 \mathrm{~K}$ and $\mathrm{J}_{1} / \mathrm{k}_{\mathrm{B}}$ $=-17.4 \mathrm{~K}$ are obtained. If this step is repeated, $\mathrm{J}_{1}$ and $\mathrm{J}_{2}$ converge to constant values for $\mathrm{J}_{1}$ and $\mathrm{J}_{2}$. The last values are $\mathrm{J}_{1} / \mathrm{k}_{\mathrm{B}}=-17 \mathrm{~K}$ and $\mathrm{J}_{2} / \mathrm{k}_{\mathrm{B}}=-101 \mathrm{~K}$. Here, $\mathrm{J}_{1}$ and $\mathrm{J}_{2}$, according to Green's function theory are different from the results according to the molecular field theory. However, Green's function approximation agrees well with the experimental 
values from the spin-wave dispersion curve, and Raman scattering. Fig. 2.4 shows the relations of $k_{B} T_{N} / J_{2}$ vs. $J_{1} / J_{2}$ which calculated by Green's function theory for FCC antiferromagnetic ordering of type II and $\mathrm{S}=1$ case [Bartel et al, 1971]. This calculation is only valid for FCC antiferromagnetic ordering type II and $\mathrm{S}=1$ case such as $\mathrm{NiO}$. Although Green's function is complicated for the $\mathrm{S} \neq 1$ case, many people have already solved $J_{1}$ and $J_{2}$ for the other compounds using Green's function approximation. The results are shown in Table 2.2. 


\begin{tabular}{|c|c|c|c|c|}
\hline \hline & \multicolumn{2}{|c|}{ By Molecular field theory } & \multicolumn{2}{c|}{ By Green`s function theory } \\
\hline Compounds & $-\mathrm{J}_{1} / \mathrm{k}_{\mathrm{B}}$ & $-\mathrm{J}_{2} / \mathrm{k}_{\mathrm{B}}$ & $-\mathrm{J}_{1} / \mathrm{k}_{\mathrm{B}}$ & $-\mathrm{J}_{2} / \mathrm{k}_{\mathrm{B}}$ \\
\hline $\mathrm{MnO}$ & 7.1 & 3.3 & 5 & 5.5 \\
\hline $\mathrm{FeO}$ & 7.8 & 8.3 & -0.9 & 8.0 \\
\hline $\mathrm{CoO}$ & 1.3 & 19.5 & 2.8 & 13.7 \\
\hline $\mathrm{NiO}$ & 81.9 & 65.4 & 17.0 & 101.0 \\
\hline$\alpha-\mathrm{MnS}$ & 5.5 & 5.9 & 3.5 & 6.4 \\
\hline \hline
\end{tabular}

Table 2.2 shows the difference between $\mathrm{J}_{1}$ and $\mathrm{J}_{2}$, determined by the molecular field theory and Green's function theory. 


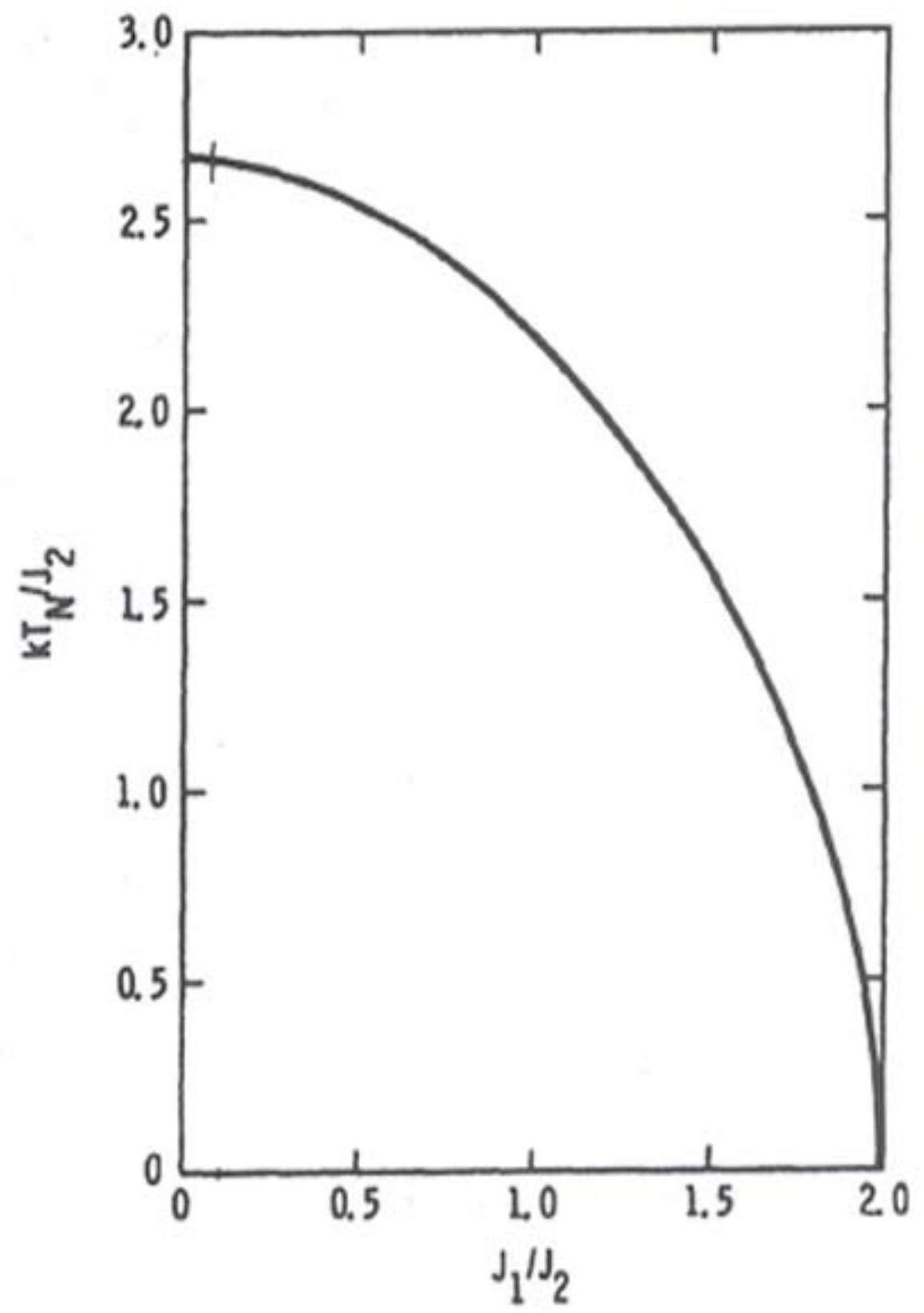

Fig. 2.4 shows the relation between $\mathrm{k}_{\mathrm{B}} \mathrm{T}_{\mathrm{N}} / \mathrm{J}_{2}$ and $\mathrm{J}_{1} / \mathrm{J}_{2}$ calculated by Green's function theory for the FCC antiferromagnetic ordering of type II and the $\mathrm{S}=1$ case [Bartel et al, 1971]. 


\subsection{Measurement of $\mathrm{J}_{1}$ and $\mathrm{J}_{2}$ for $\mathrm{NiO}$ by other methods}

There are several ways to measure $J_{1}$ and $J_{2}$ experimentally such as measurement of spin - wave dispersion by Inelastic Neutron Scattering techniques [Hutchings et al, 1972] and Raman scattering data [Dietz et al, 1971].

\begin{tabular}{ccc}
\hline \hline Experiment & $-\mathrm{J}_{1} / \mathrm{k}_{\mathrm{B}}$ & $-\mathrm{J}_{2} / \mathrm{k}_{\mathrm{B}}$ \\
\hline Neutron work & -8.0 & 111 \\
& & \\
Raman Scattering & small & 107 \\
& & 65 \\
Molecular field theory & & \\
(from $\theta$ and $\mathrm{T}_{\mathrm{N}}$ ) & 82 & 66 \\
& & \\
Molecular field theory & & \\
(from $\mathrm{T}_{\mathrm{N}}$ and $\chi$ ) & 52 & 101 \\
& & \\
\hline \hline
\end{tabular}

Table 2.3 shows the difference of $\mathrm{J}_{1}$ and $\mathrm{J}_{2}$ determined by several methods to compare with the molecular field theory. 


\section{CHAPTER III}

\section{Synthesis and Structural Properties}

\subsection{Synthesis}

The NiO nanoparticles were synthesized chemically through the sol-gel technique [Pierre et al. 1998], which is used to produce metal oxides. The synthesis procedure consisted of four steps: the reaction of the precursor to form sol, gelation, drying, and annealing. The first step is to make a nickel hydroxide $\mathrm{Ni}(\mathrm{OH})_{2}$ precursor called a sol. To get this, a completely dissolved nickel nitrate $\mathrm{Ni}\left(\mathrm{NO}_{3}\right)_{2} \cdot 6 \mathrm{H}_{2} \mathrm{O}$ aqueous solution and an aqueous sodium hydroxide $\mathrm{NaOH}$ are mixed, making $\mathrm{pH}=12$. The reaction is

$$
\mathrm{Ni}\left(\mathrm{NO}_{3}\right)_{2} \cdot 6 \mathrm{H}_{2} \mathrm{O}+2 \mathrm{NaOH} \rightarrow \mathrm{Ni}(\mathrm{OH})_{2}+2 \mathrm{NaNO}_{3}+6 \mathrm{H}_{2} \mathrm{O} \text {. }
$$

The reason for the $\mathrm{pH}=12$ is that for this $\mathrm{pH}$ only, the XRD (x-ray diffraction) pattern of the product has no impurities, except the pattern of powder $\mathrm{Ni}(\mathrm{OH})_{2}$. This means that when the $\mathrm{pH}=12$, the two chemicals have completely reacted. The $\mathrm{Ni}(\mathrm{OH})_{2}$ precursor was centrifuged to remove any liquid. During this activity, the sample was washed many times with distilled water to remove any impurities. It is called gelation. The gel was left at room temperature overnight to dry the gel. It yielded powder form of $\mathrm{Ni}(\mathrm{OH})_{2}$ by grinding the dried gel. $\mathrm{Ni}(\mathrm{OH})_{2}$ was determined to decompose to $\mathrm{NiO}$ at $200 \mathrm{C}$ using thermogravimetric measurements according to the equation,

$$
\mathrm{Ni}(\mathrm{OH})_{2} \rightarrow \mathrm{NiO}+\mathrm{H}_{2} \mathrm{O} \text {. }
$$

To obtain $\mathrm{NiO}$ particles with different sizes, the obtained powder samples are annealed at various temperatures. Using X-ray diffraction (in section 3.3), it was found that particles with the sizes of 5, 7, 8, 12,16, 20, and $41 \mathrm{~nm}$ were obtained by annealing at the temperatures of $523,573,623,673,723,773$, and $873 \mathrm{~K}$ respectively. Here, each sample will be known by its nominal size, determined by XRD.

$\mathrm{NiO}$ nanoparticles have been studied by several groups [Jacobs et al, 1963, Richardson et al, 1991 and references therein]. Although NiO nanoparticles are strongly aggregated, these authors did not consider the effect of interparticle interactions which affect magnetic properties. Bødker et al [Bødker et al, 2000] proposed methods to reduce the interparticle interactions by particle coating. We employed this coating method to 
verify the effect of interparticle interactions. Each size of $\mathrm{NiO}$ powder was milled using a ball mill with a tungsten carbide vial and four 7/16 inch tungsten carbide balls made by Spex. These particles, when ground into a fine powder, were dispersed in $0.01 \mathrm{M} \mathrm{HNO}_{3}$,

given ultrasonic treatment, and centrifuged to get dispersed particles. To fix this dispersion, a small amount of Oleic Acid was added. The samples were air dried overnight. These coated samples were investigated in many experiments such as $\mathrm{XRD}$, TEM, and SQUID for magnetic analysis and their properties compared with those of uncoated samples.

\subsection{Transmission Electron Microscopy (TEM)}

We investigated the particle size distribution and morphology through the use of Transmission Electron Microscopy (TEM) for various sized particles of both uncoated and coated $\mathrm{NiO}$ nanoparticles. The particles here are labeled according to the average size determined by XRD. TEM studies reported here were kindly done by Dr. John Bonevich (National Institute of Standards and Technology, Gaithersburg, MD) on samples prepared by us for the TEM studies.

A TEM is capable of providing crystallographic information and also produce images of nanoparticle surfaces. In a TEM, the electrons from a source enter the sample and are scattered as they pass through it. These particles are then focused by an objective lens and are amplified by a magnifying lens, finally producing the desired image [Poole et al. 1990]. The results from the TEM micrographs are really important and interesting since for $\mathrm{D}<10 \mathrm{~nm}$, the particles are primarily in the form of nanorods. However for $\mathrm{D}$ $>10 \mathrm{~nm}$, the particle shapes become more rounded. In none of the earlier studies of $\mathrm{NiO}$ nanoparticles prepared by the sol-gel methods, such features have been reported [Bødker et al. 2000, and Tiwari et al. 2005].

Fig. 3.1 shows the results of TEM measurements for the particle size, $D=5 \mathrm{~nm}$ of both the uncoated (a) and coated (b) cases. In Fig. 3.1 (a), the overall shape of the particles looks like entangled hair and the rods are very strongly aggregated. Thus, efforts were made to figure out the diameter of all rods. The coated nanorods of particle size 5 $\mathrm{nm}$ tend to be less entangled and more straight and so were easier to measure. We picked 
rods as many as we could to measure the diameter and the obtained average diameter was approximately $1.7 \mathrm{~nm}$ for both the uncoated and coated nanorods.

For the samples of nominal XRD sizes of 7 (7) nm and 8 (11) nm, there are mixed shapes of ellipses and rods in uncoated (coated) cases. The uncoated cases shown in Fig 3.2 (a), (b) and Fig. 3.3 (a), (b) were so hazy that the exact size could not be read, especially for the $7 \mathrm{~nm}$ cases since the rods lay one upon another. In Table 3.1, we have summarized the sizes of the particles as determined from TEM and XRD measurements. It is noted that the diameters of the nanorods for different particles are smaller than the sizes measured by the XRD. However, it turns out that volume of the nanorods nearly equals the volume of an equivalent sphere with diameter equal to the size of particles determined by XRD. For the ellipse, the obtained sizes were 7 and $9 \mathrm{~nm}$, which were closer to the XRD results, similar to the coated 7 and $11 \mathrm{~nm}$ particles. The typical particle size distribution for the coated $7 \mathrm{~nm}$ particles is shown in Fig. 3.3 (e). Through the TEM pictures of the uncoated $8 \mathrm{~nm}$ and the coated $11 \mathrm{~nm}$ particles, we can see that fewer particles are in the form of nanorods compared to the 5 and $7 \mathrm{~nm}$ samples. Fig. 3.2 (c) represents the uncoated $12 \mathrm{~nm}$ particles showing the reduced quantity of the nanorods and rods that look like a pill with uniform sides. For the uncoated $16 \mathrm{~nm}$ particles and coated $17 \mathrm{~nm}$ particles, their TEM show that the spheres and ellipses are mixed, but there are no rods. It is shown in Fig. 3.2 (d) and Fig. 3.3 (c). For the uncoated $20 \mathrm{~nm}$ and $41 \mathrm{~nm}$ particles and the coated $20 \mathrm{~nm}$ sample, TEM pictures are shown in Fig. 3.2 (e), (f) and Fig. 3.3 (d) respectively. As the particle size becomes larger, the shape of the particles changes to a near sphere and, so it is much easier to measure the particle size, now that the particles are much more dispersed. Thus, we could determine the particle size distribution statistically. These bar graphs are shown in Fig 3.2 (g) and Fig. 3.3 (f) for the uncoated $20 \mathrm{~nm}$ and coated $27 \mathrm{~nm}$ samples.

It is evident that the shape of nanoparticle is transformed from a rod to a sphere by using a higher annealing temperature. For the particle size D $>10 \mathrm{~nm}$, most of the rods have disappeared and are replaced by nearly spherical particles. The particle sizes are estimated from these spheres and ellipses which are very close to the size obtained by XRD. 


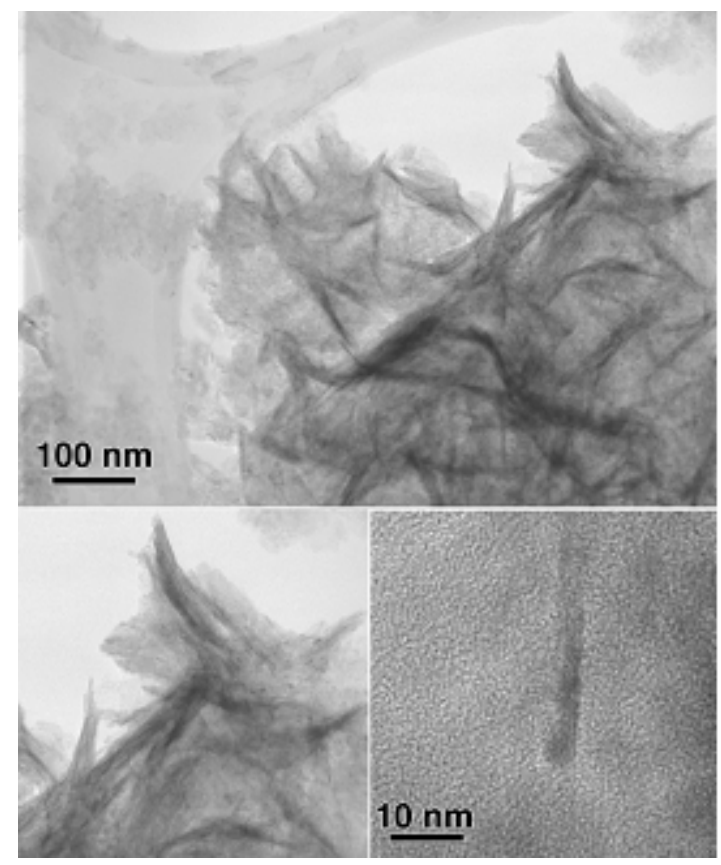

(a) uncoated

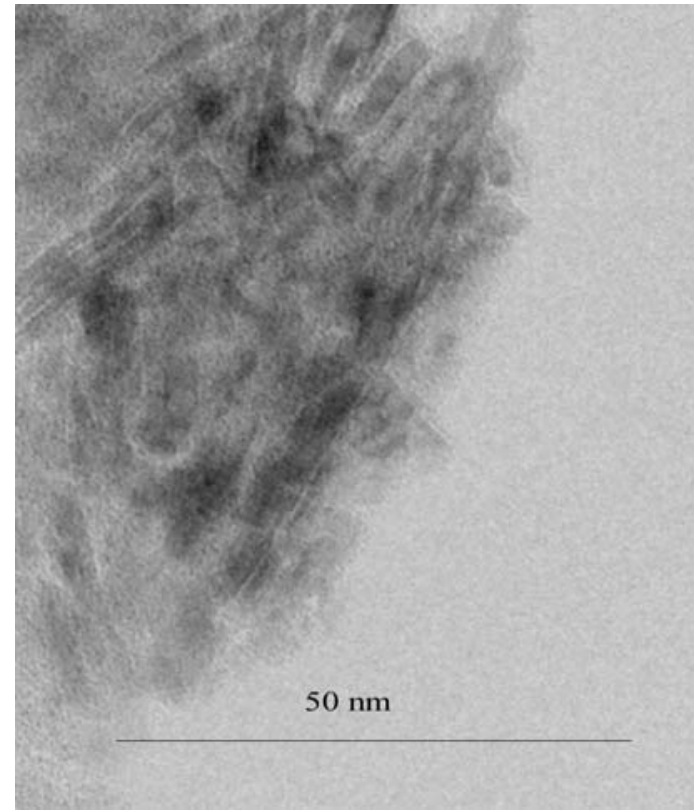

(b) coated

Fig. 3.1 TEM pictures of particle size $\mathrm{D}=5 \mathrm{~nm}$ for uncoated (a) and coated (b) cases. 


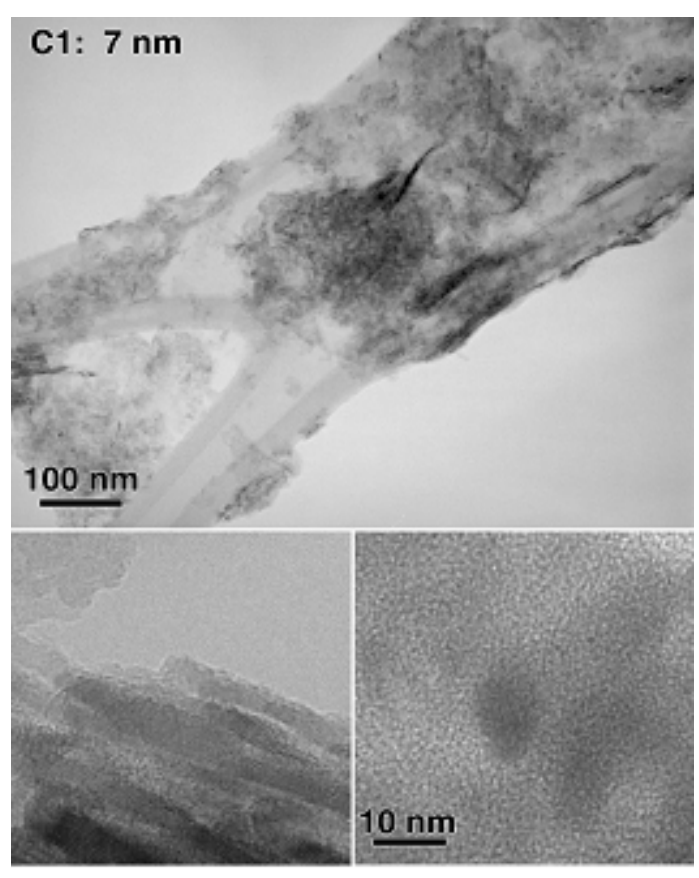

(a)

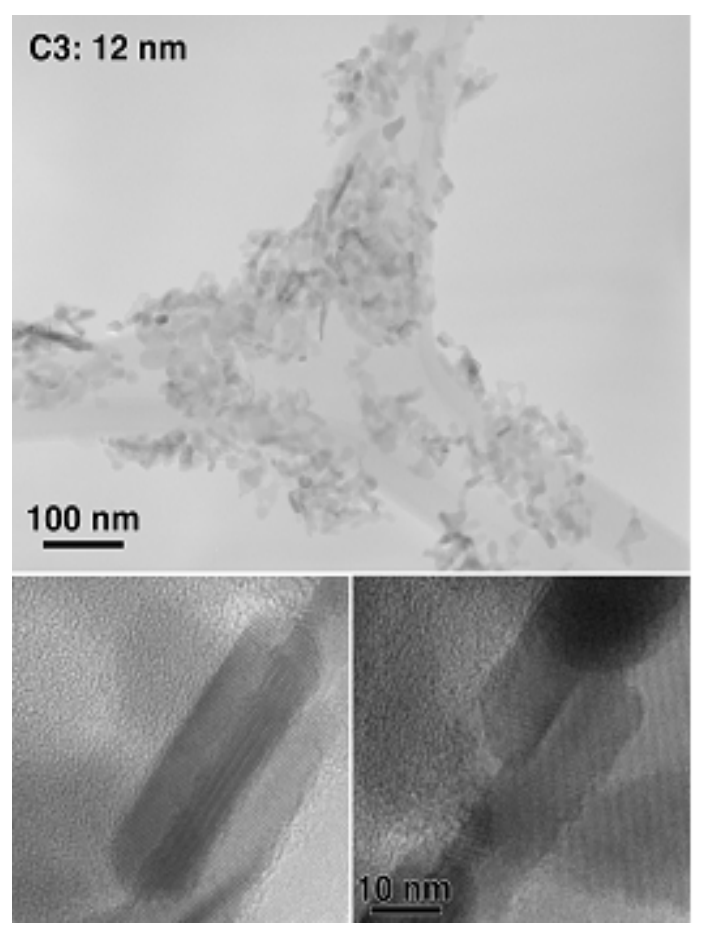

(c)

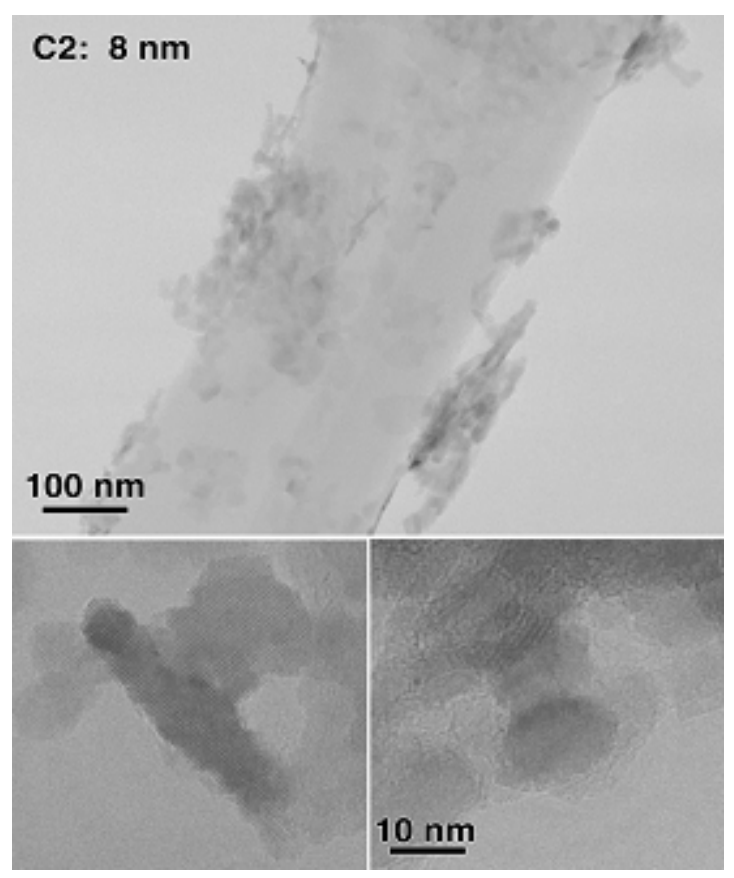

(b)

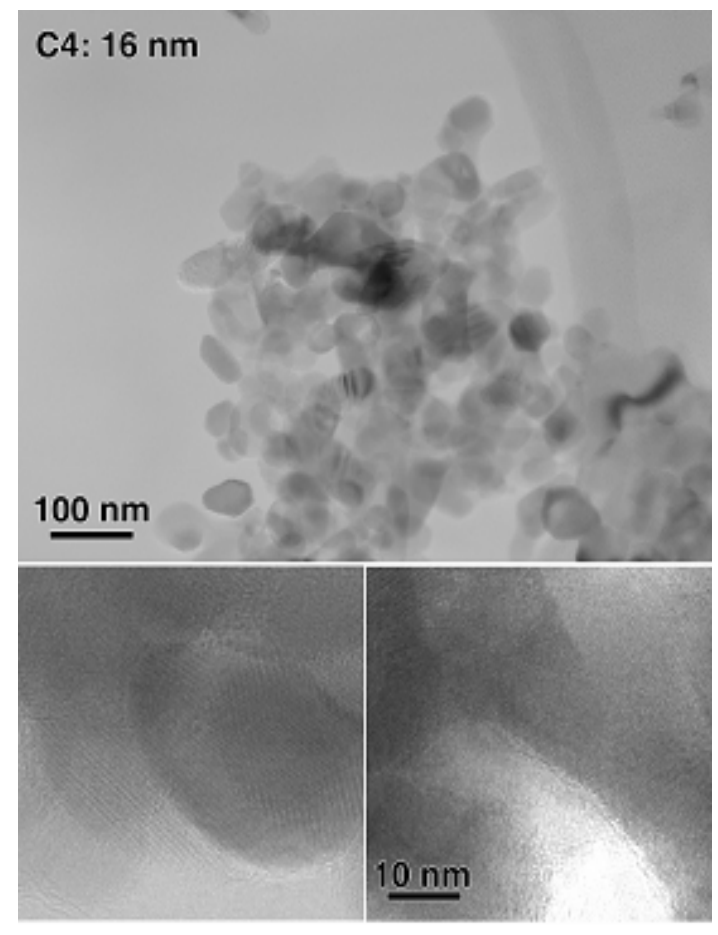

(d) 


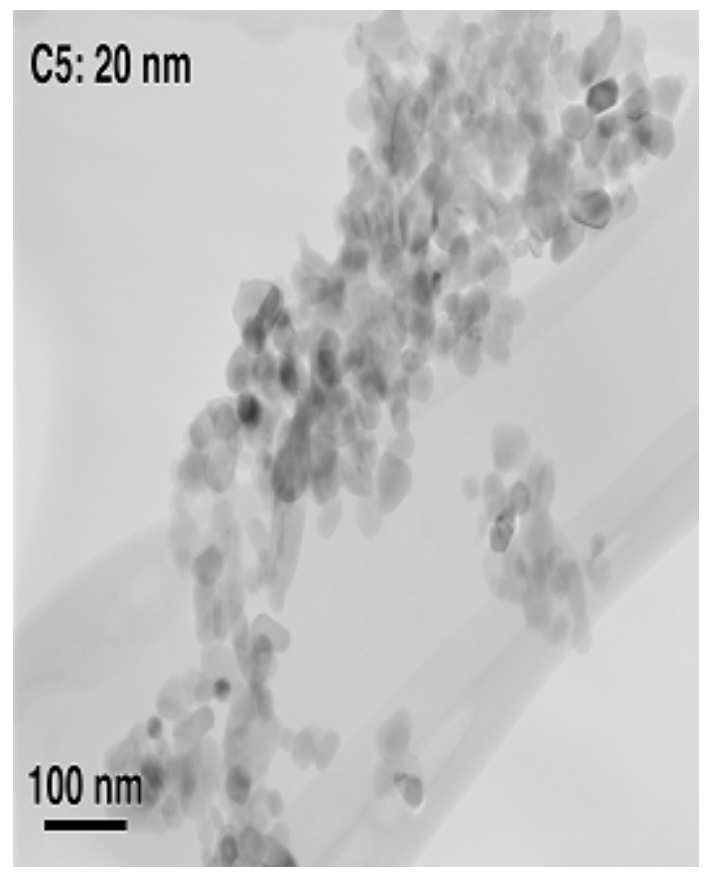

(e)

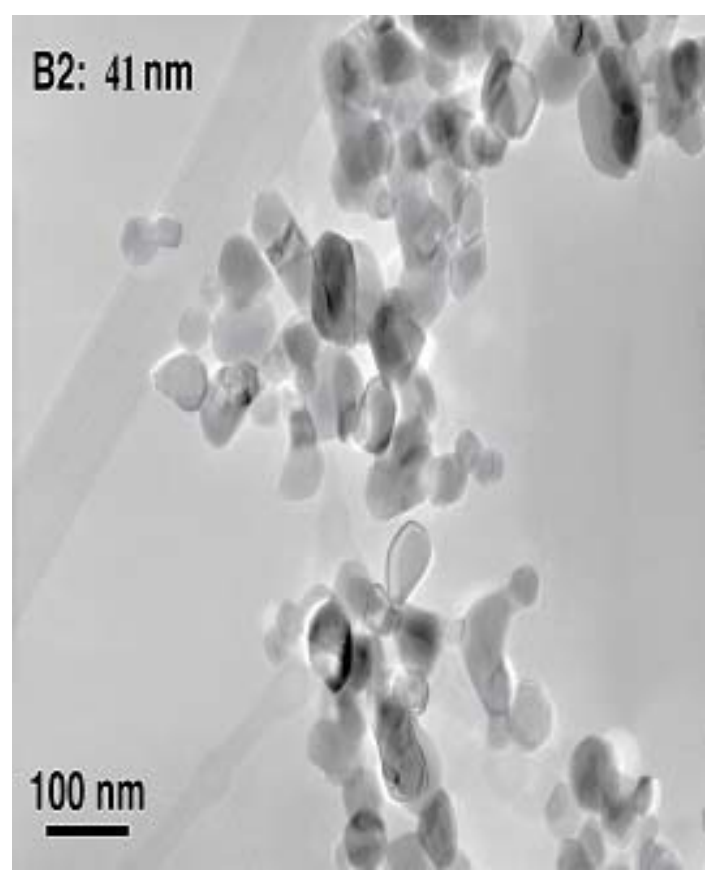

(f)

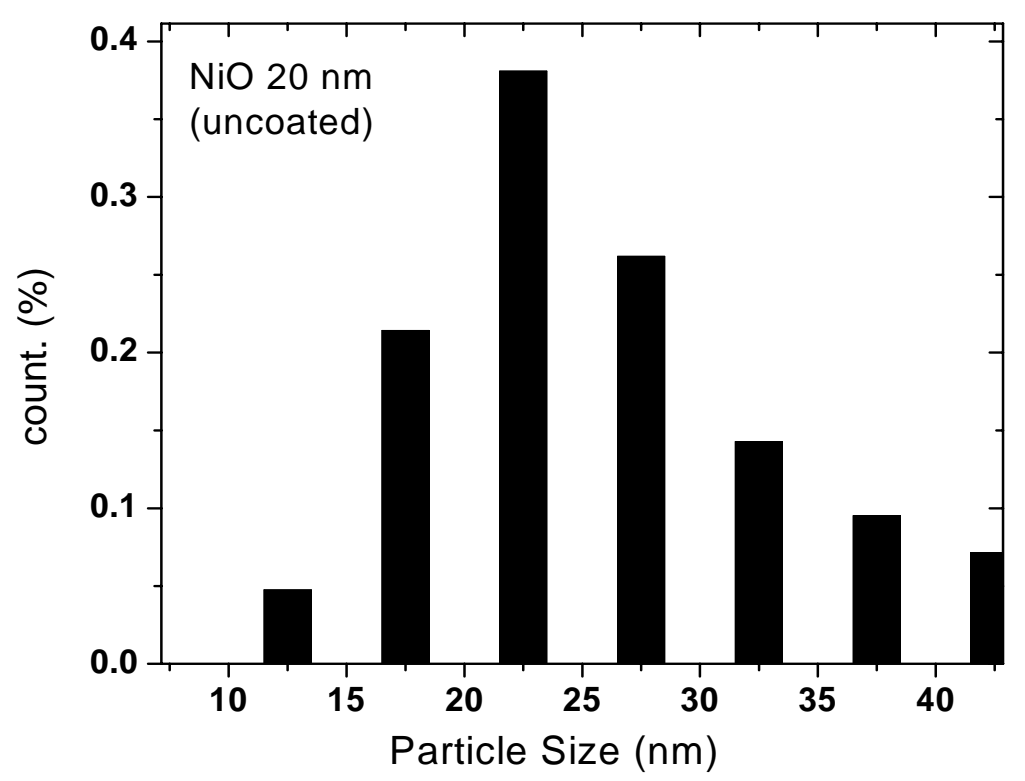

(g)

Fig. 3.2 Transmission electron microscopy (TEM) images of $\mathrm{NiO}$ 7, 8, 12, 1620 and 41 $\mathrm{nm}$ samples represented in (a), (b), (c), (d), (e) and (f) respectively. 


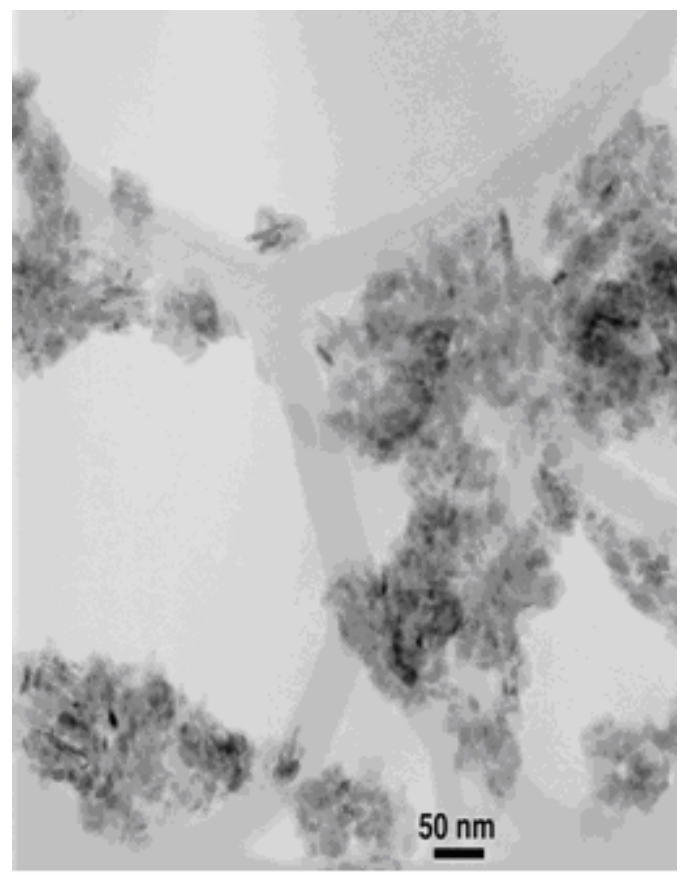

(a)

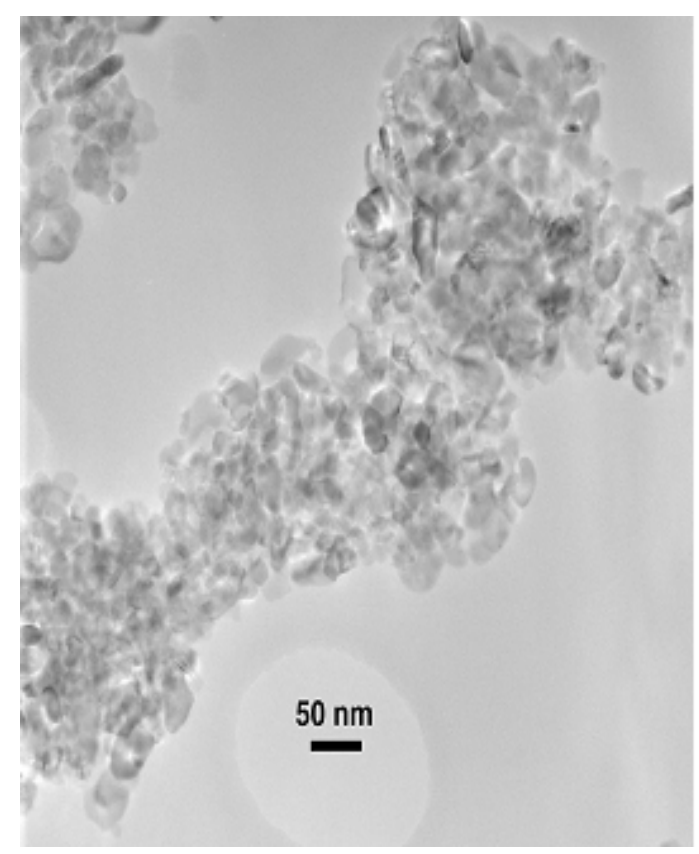

(c)

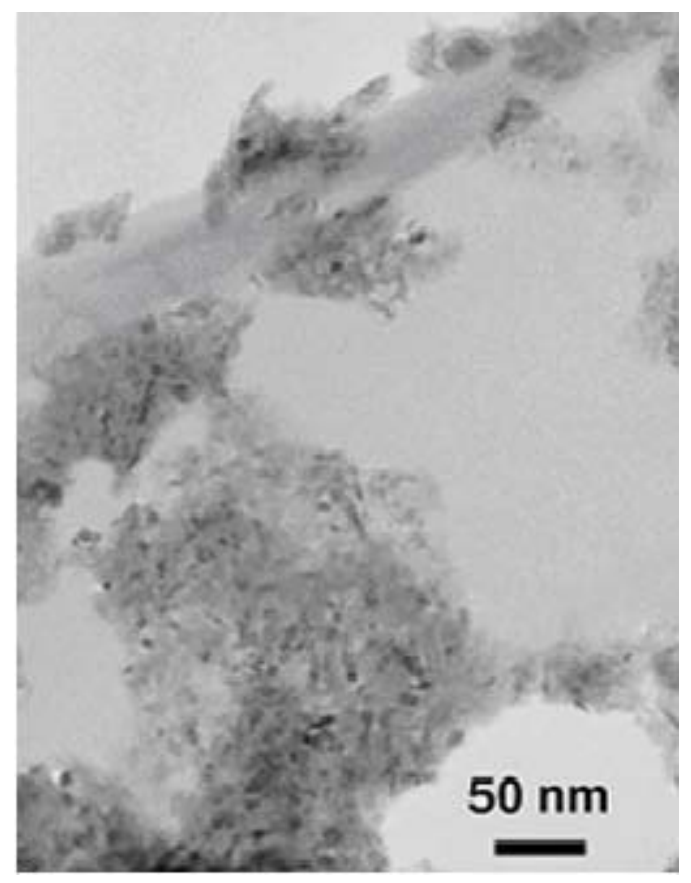

(b)

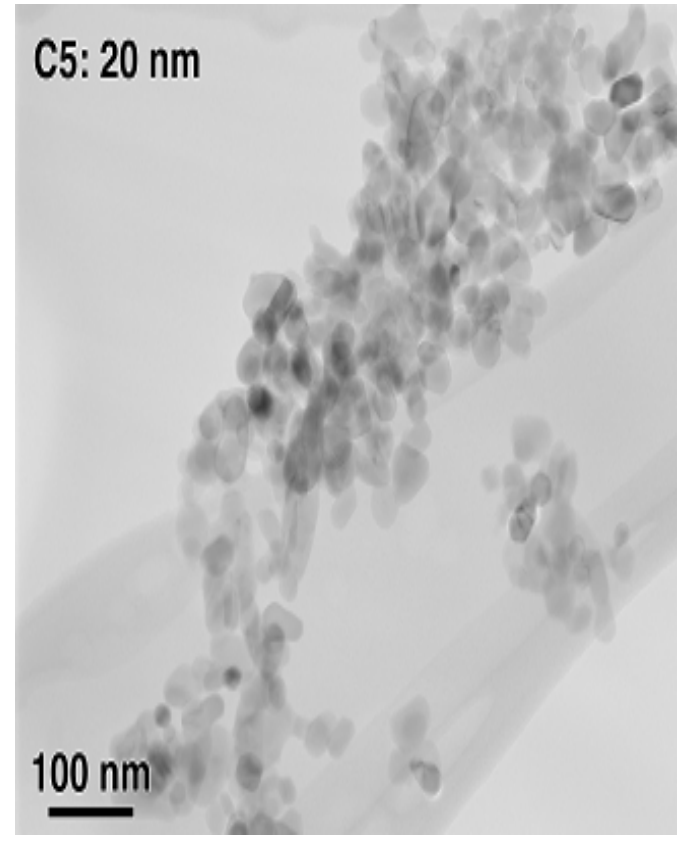

(d) 


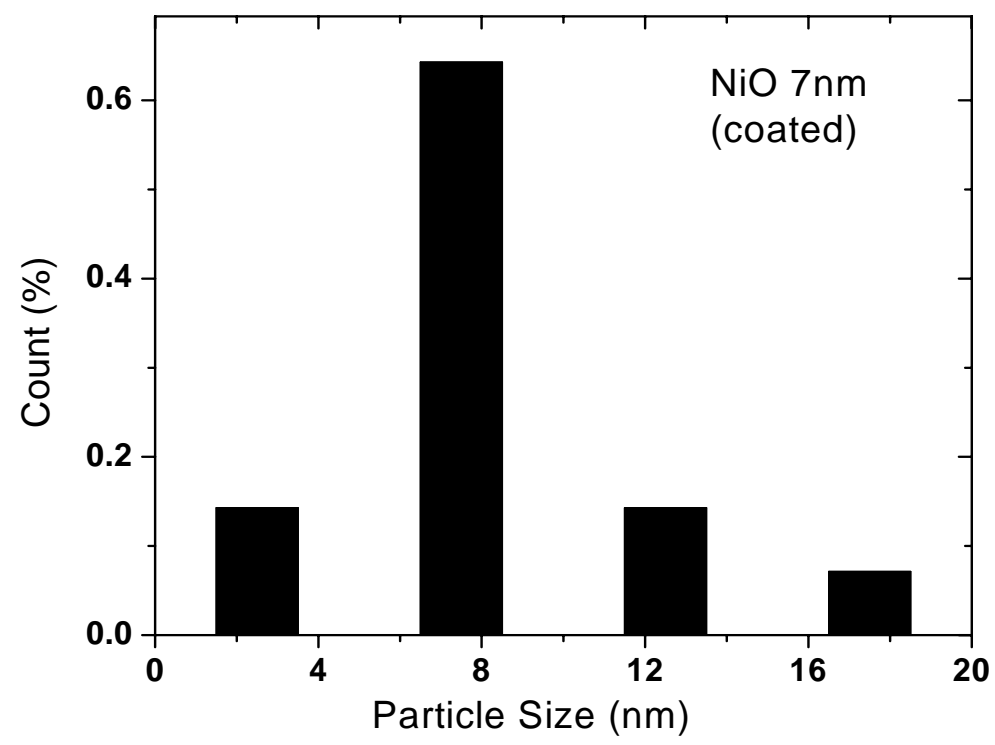

(e)

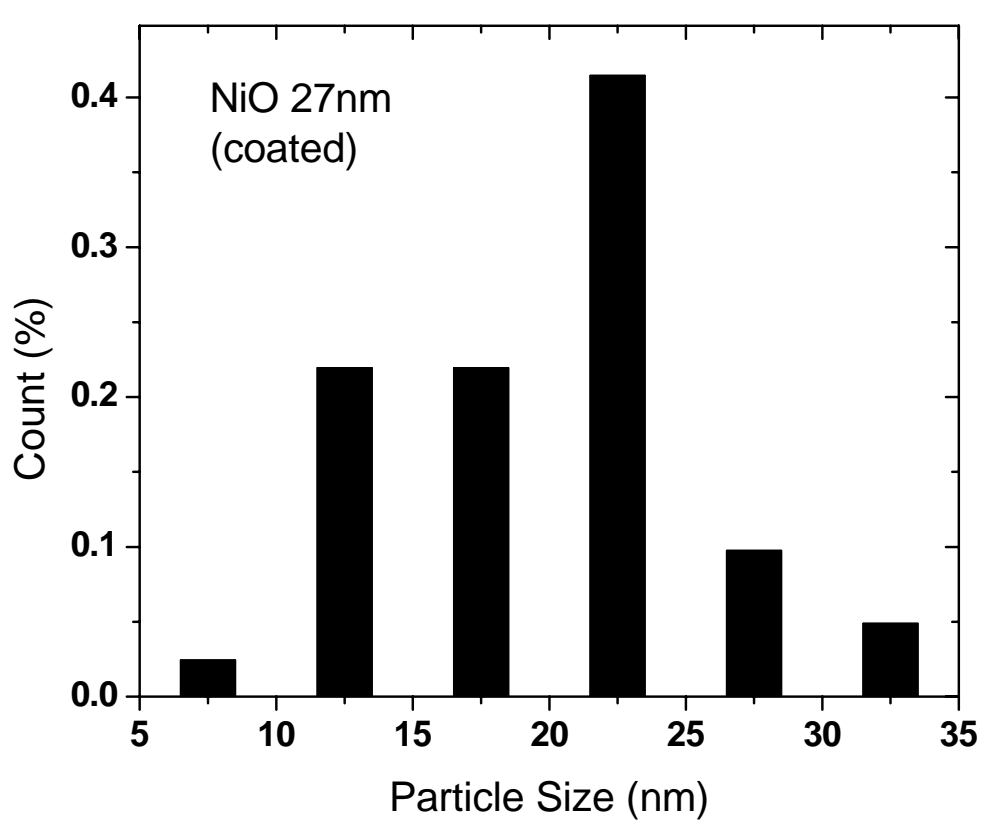

(f)

Fig. 3.3 Transmission electron microscopy (TEM) images for coated 7, 8, 17, and $27 \mathrm{~nm}$ samples represented in (a), (b), (c) and (d) respectively. 


\subsection{X-ray Diffraction (XRD)}

$\mathrm{X}$-ray diffraction analysis was performed on all the samples using a Rigaku Diffractometer and monochromatic radiation $\mathrm{CuK} \alpha$. This diffractometer was used to determine the unknown spacing of the crystal planes with the known wavelength ( $\lambda=$ $1.5418 \AA$ ) of X-rays using a powder sample. In our experiments on $\mathrm{NiO}$, voltage applied to the target was $40 \mathrm{kV}$, filament current was set to $30 \mathrm{~mA}$ and the scan range of $2 \theta$ was $20^{\circ}$ to $100^{\circ}$ at the speed $0.06^{\circ} / 5 \mathrm{sec}$. Small amounts of sample annealed at various temperatures were placed in the middle of glass sample holder and pressed gently by hand to make a smooth surface. Then, one to two drops of ethanol were added onto the spot which the sample was located and dried in air. The sample holder with the sample was placed vertically in the diffractometer. Fig. 3.4 shows the results for each sample. With these results, we matched the phase of each sample to the phase of the pure $\mathrm{NiO}$ using the software JADE and JCPDC data base. There were no significant impurities. Any line shift for different annealing temperatures is negligible and the Bragg lines of small particle are broadened due to the small crystalline size. These results are discussed next. 

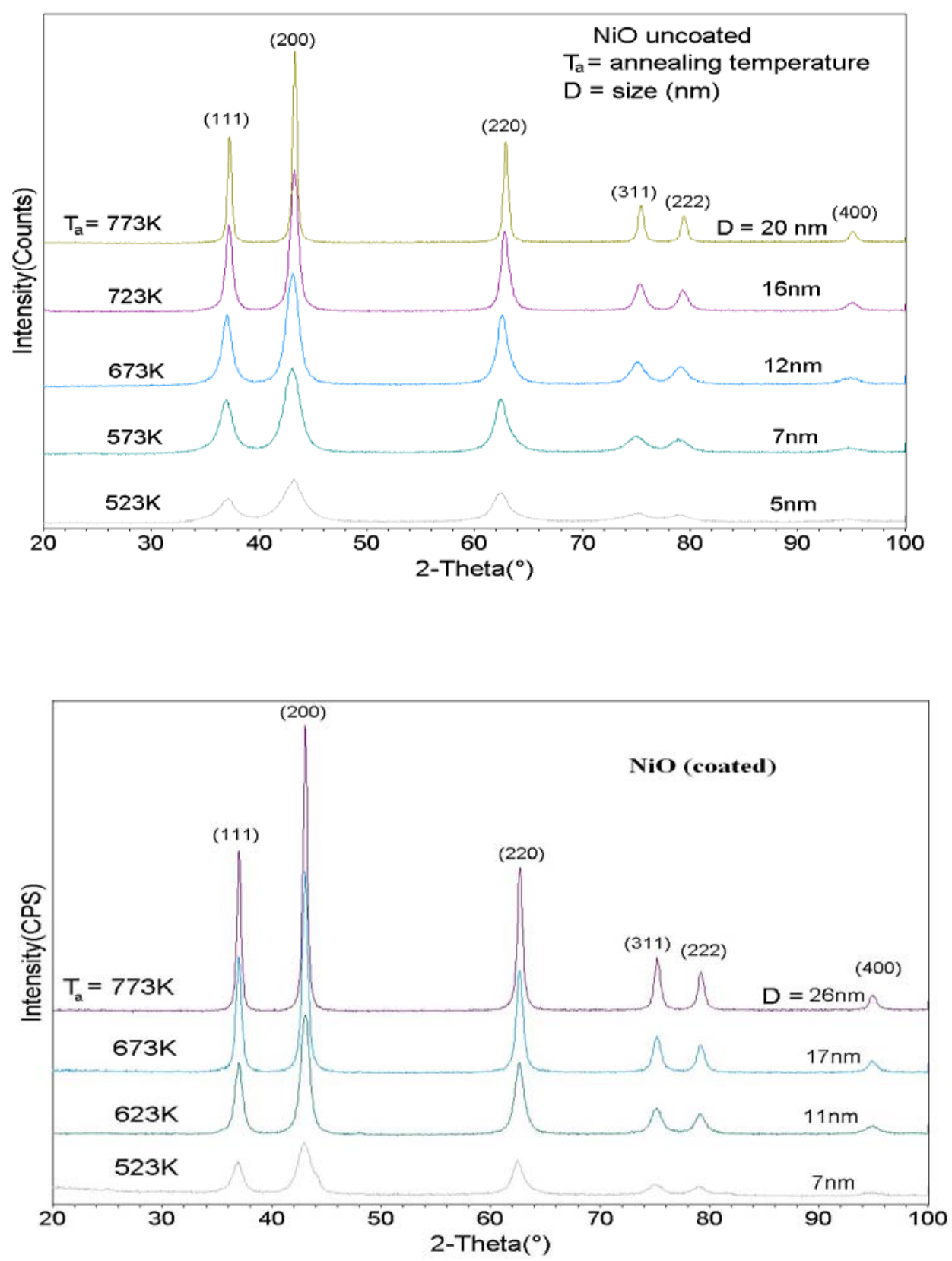

Fig. 3.4 shows XRD patterns for all particle size annealed at various temperatures for uncoated and coated samples. 


\subsection{Particle size broadening from XRD}

XRD patterns contain significant information about the material: the Miller indices, the unit cell dimensions and especially the particle size and strain and so on. In the previous section, we identified the patterns by comparing with the standard $\mathrm{NiO}$ phase in JADE. The identified phase in JADE gives us basic information of indices and structure of materials directly. The NiO indices and structure observed from JADE were (111), (200), (311), (222), and (400) and a sodium chloride structure. Important information from XRD patterns is the determination of particle size and strain. For the powder sample, the analysis of XRD patterns were fit to the modified Scherrer equation,

$$
\beta \cos \theta=0.9 \lambda / \mathrm{D}+\eta \sin \theta
$$

where $D$ is the particle size, $\eta$ is the strain and $\beta$ is full-width at half maximum of a diffraction peak at the angle $2 \theta$.

To understand the broadening due to particle size $\mathrm{D}$, we follow the procedure outlined in Klug et al. [1974]. First the Bragg's law for X-ray diffraction is given by (Figs. 3.5 and 3.6):

$$
2 \mathrm{~d} \sin \theta_{\mathrm{B}}=\mathrm{n} \lambda .
$$

According to Eq. (3.4), at $\theta=\theta_{\mathrm{B}}$, the path difference between rays refracted from consecutive planes is $\lambda$ whereas at all other angles, the phases of the scattered rays are such as to cancel out completely for an infinite crystal. This leads to a $\delta$-function at $\theta=$ $\theta_{\mathrm{B}}$.

When a crystal is of finite size $\mathrm{t}=\mathrm{md}$ with $\mathrm{m}+1$ plains (Fig. 3.6), then for some angles near $\theta_{\mathrm{B}}$ complete cancellation of the beam only occurs at $2 \theta_{1}$ and $2 \theta_{2}$ slightly different from $2 \theta_{\mathrm{B}}$. We define the width $\mathrm{B}$ as

$$
\mathrm{B}=1 / 2\left(2 \theta_{1}-2 \theta_{2}\right)=\theta_{1}-\theta_{2}
$$

where $\left(\theta_{1}-\theta_{2}\right)$ is the angular spread which gives rise to the width. Then the equations for the angles $\theta_{1}$ and $\theta_{2}$ are 


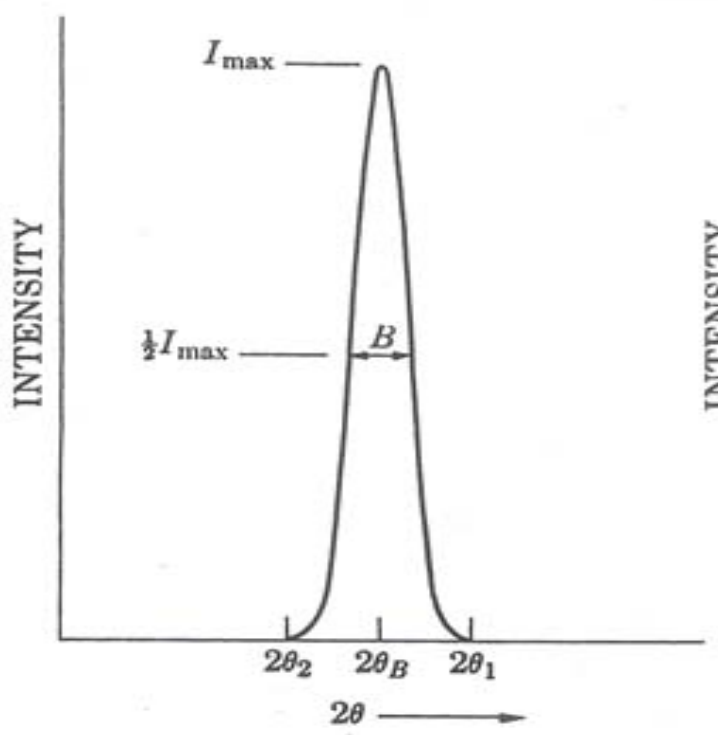

(a)

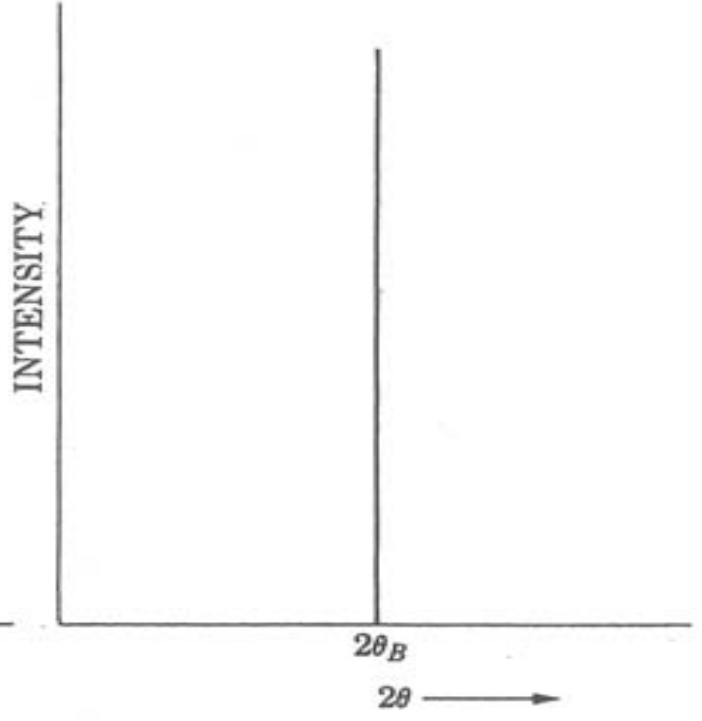

(b)

Fig. 3.5 indicates the diffracted beam for actual case (a) and ideal case (b).

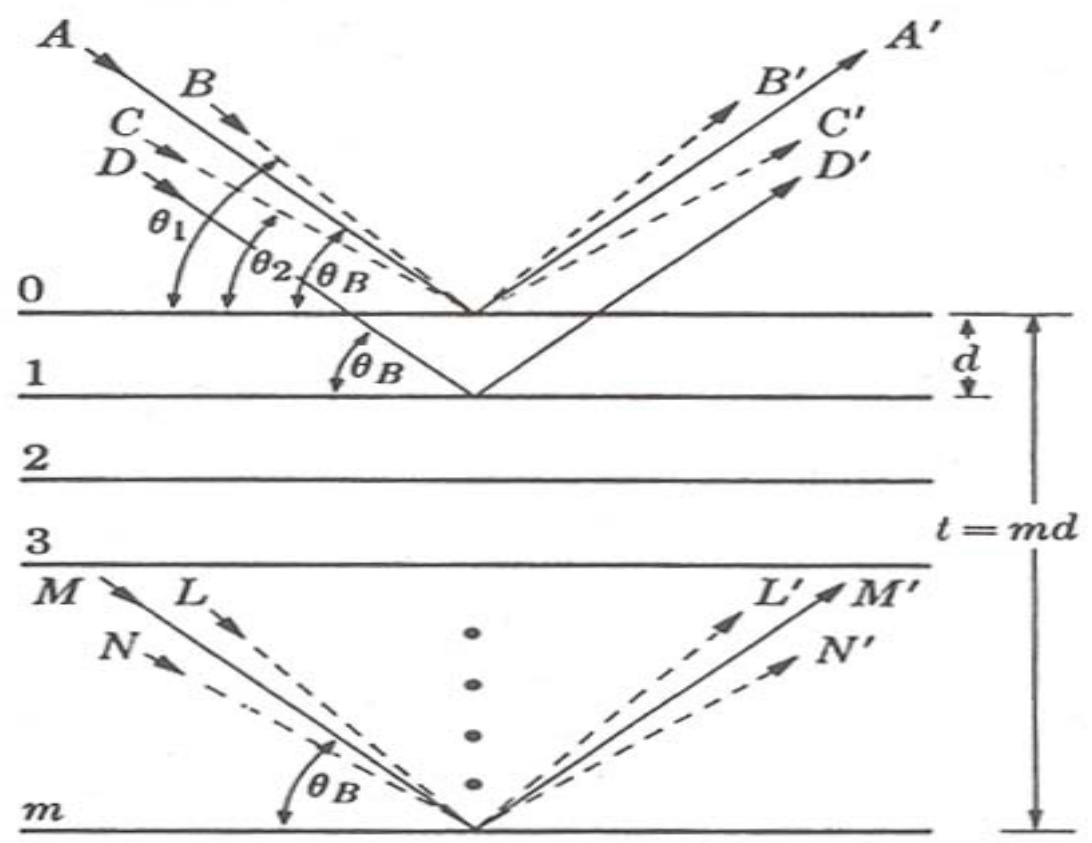

Fig. 3.6 Diagram shows the effect of crystal size on diffraction. [Klug et al]. 


$$
\begin{aligned}
& 2 \mathrm{t} \sin \theta_{1}=(\mathrm{m}+1) \lambda \\
& 2 \mathrm{t} \sin \theta_{2}=(\mathrm{m}-1) \lambda
\end{aligned}
$$

By subtraction in Eq (3.6),

$$
\begin{gathered}
\mathrm{t}\left(\sin \theta_{1}-\sin \theta_{2}\right)=\lambda \\
2 \mathrm{t} \cos \left\{\left(\theta_{1}+\theta_{2}\right) / 2\right\} \sin \left\{\left(\theta_{1}-\theta_{2}\right) / 2\right\}=\lambda
\end{gathered}
$$

As $\theta_{1}$ and $\theta_{2}$ are both very close to $\theta_{\mathrm{B}}$, approximately, $\theta_{1}+\theta_{2}=2 \theta_{\mathrm{B}}$ and $\sin \left\{\left(\theta_{1}-\theta_{2}\right) / 2\right\}=$ $\left\{\left(\theta_{1}-\theta_{2}\right) / 2\right\}$. Therefore,

$$
\begin{gathered}
2 \mathrm{t}\left\{\left(\theta_{1}-\theta_{2}\right) / 2\right\} \cos \theta_{\mathrm{B}}=\lambda, \\
\mathrm{t}=\lambda /\left(\mathrm{B} \cos \theta_{\mathrm{B}}\right)
\end{gathered}
$$

A more accurate form is,

$$
\mathrm{t}=0.89 \lambda /\left(\mathrm{B} \cos \theta_{\mathrm{B}}\right)
$$

Eq (3.9) is the Scherrer formula. Here t represents the particle size D. The accurate width $\mathrm{B}$ can be obtained by taking into account the instrument error due to separation of the $\mathrm{K} \alpha$ doublet. At the low angle, the separation of $\alpha_{1}$ and $\alpha_{2}$ is negligible. But at high angle, it becomes more important. The corrected width $\beta$ will be

$$
\beta_{\text {corr }}^{2}=B^{2}-b^{2}
$$

where $\mathrm{b}$ is instrumental width and depends on angle $\theta$.

When the Bragg peak of XRD is considered, we also should take into account the broadening due to strain. The effect of strain is two fold, line shift and broadening. If strain is uniform, the line is shifted from where it should be and if strain is non - uniform, there is a shape distortion resulting in the broadening of the line. The peak of metal oxide can be broadened due to stress or due to smaller particle size. Although it is not always possible to know whether the observed broadening is due to stress or to smaller particle size, through our XRD pattern and TEM studies, we found that the XRD peaks are broadened as the particle size is reduced and when we compare two TEM pictures of 5 
$\mathrm{nm}$ coated and uncoated samples, the coated sample is more straight than twisted uncoated one. Thus, the uncoated particle has a more broadened peak due to non uniform stress. This comparison is shown in Fig. 3.7. Therefore the width $\beta$ should include strain factor. The total width $\beta$ is

$$
\beta_{\text {tot }}=\beta_{\text {corr }}=\beta_{\text {crystalline }}+\beta_{\text {strain }}
$$

To calculate the broadening due to strain, let $2 \mathrm{~d}=\lambda / \sin \theta$ and differentiate both sides to yield $2 \Delta \mathrm{d}=-\mathrm{d} / \tan \theta(2 \Delta \theta)$. Thus $\beta_{\text {strain }}$ is

$$
\beta_{\text {strain }}=2 \Delta \theta=-2(\Delta \mathrm{d} / \mathrm{d}) \tan \theta \approx \eta \tan \theta
$$

where $\eta$ is strain constant.

Thus the total breadth $\beta_{\text {tot }}$ is

$$
\begin{aligned}
& \beta_{\text {tot }}=0.9 \lambda / D \cos \theta+\eta \tan \theta \\
& \beta_{\text {tot }} \cos \theta=0.9 \lambda / D+\eta \sin \theta
\end{aligned}
$$

Eq (3.13) is the modified Scherrer relation. From the graph $\beta_{\text {tot }} \cos \theta_{B}$ vs. $\sin \theta$ (Fig. 3.8), the intercept at $\sin \theta=0$, yields $D=0.89 \lambda /\left(\beta_{\text {tot }} \cos \theta_{B}\right)$ and the slope of the lines yields $\eta$. Thus, for the uncoated samples, the resulting particle sizes are 4.8, 7.0, 8.5, 12.0, 16.0, 20.0, and $41.0 \mathrm{~nm}$ annealed at temperatures 523, 573, 623, 673, 723, 773 and $873 \mathrm{~K}$ respectively. Similarly for the coated particles, sizes 5.3, 7.0, 11.0, 17.0and $27.0 \mathrm{~nm}$ are obtained for the annealing temperature 523, 573, 623, 673 and $773 \mathrm{~K}$. These results are shown in Table 3.1.

From the plot of strain $\eta$ vs. particle size D shown in Fig. 3.9, there are large error bars for particle sizes $<10 \mathrm{~nm}$. Above $10 \mathrm{~nm}$, the strain for both uncoated and coated particles does not show any particle size dependence. This corresponds to the pattern of transition of the shape of the particles from rods to spheres. Significant reduction of the strain for the coated particles for $\mathrm{D}<10 \mathrm{~nm}$, is observed as compared to the values of the uncoated particles. Note that sizes here are average sizes determined by $\mathrm{XRD}$ in terms of diameter of a sphere with equivalent volume. 


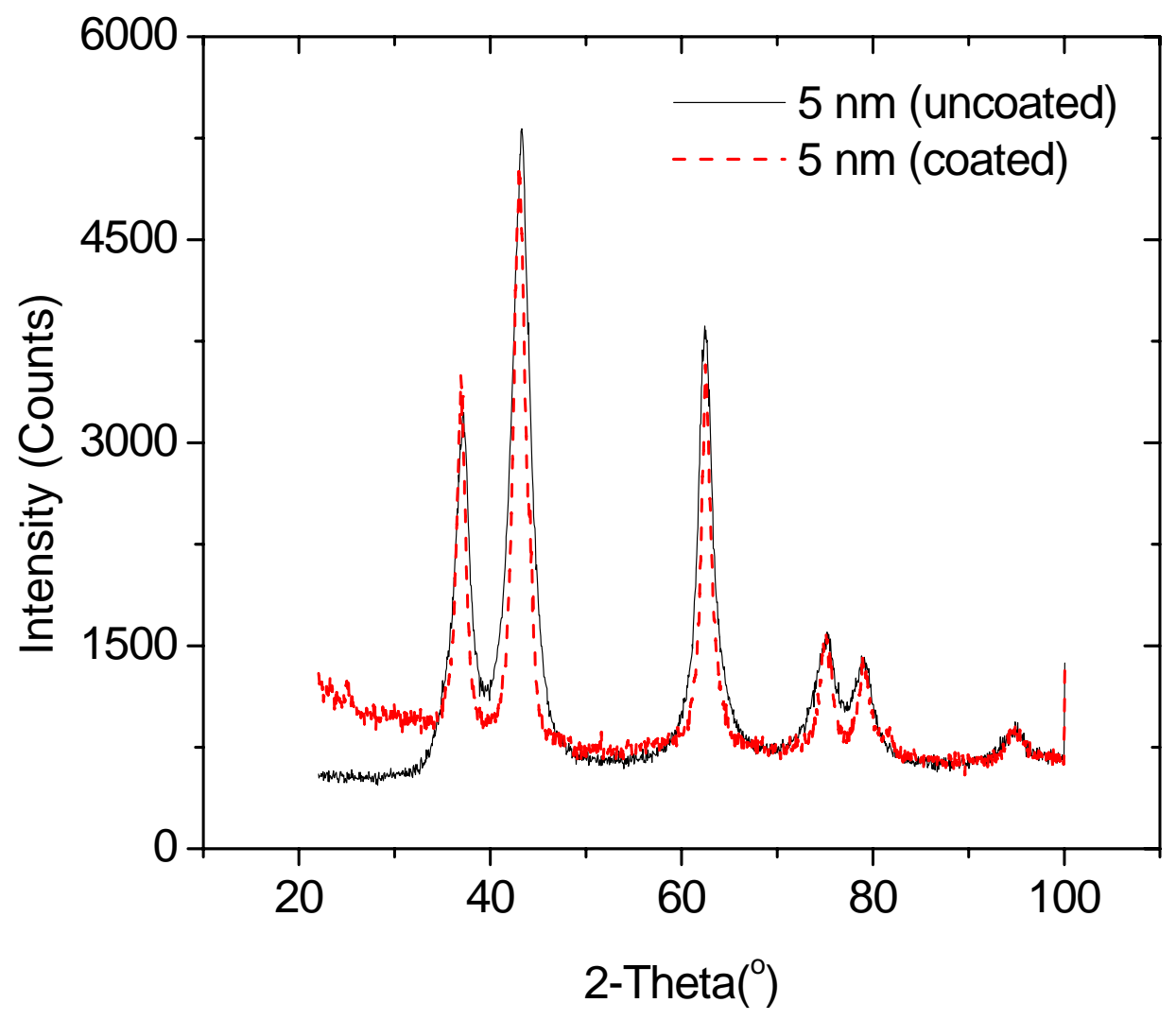

Fig. 3.7 The comparison of the XRD patterns broadening for the coated and uncoated 5 $\mathrm{nm}$ particles. 


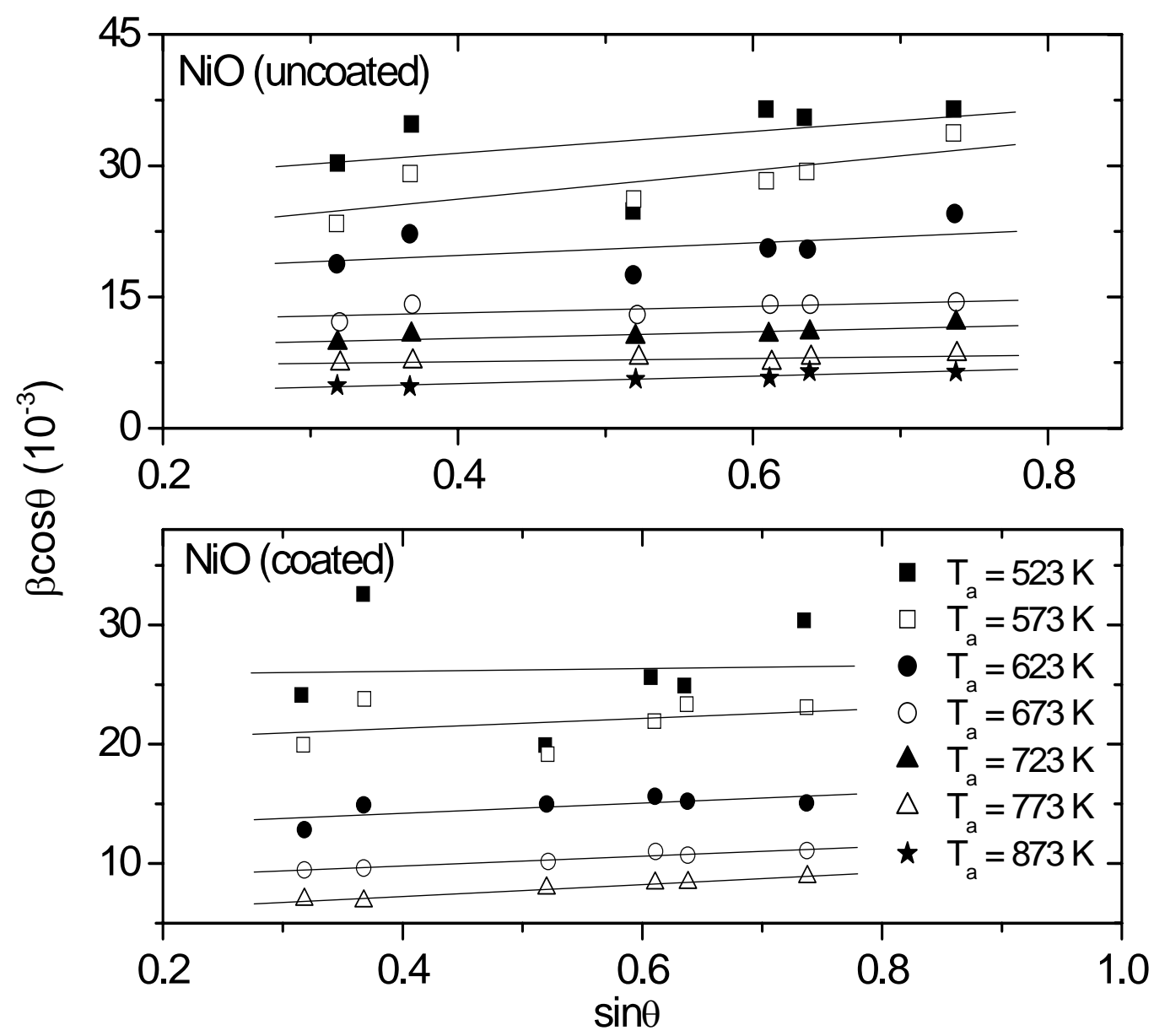

Fig. 3.8 Plots of $\beta \cos \theta$ vs. $\sin \theta$ for different samples annealed at the various temperatures and the analysis for the uncoated and coated particles. 


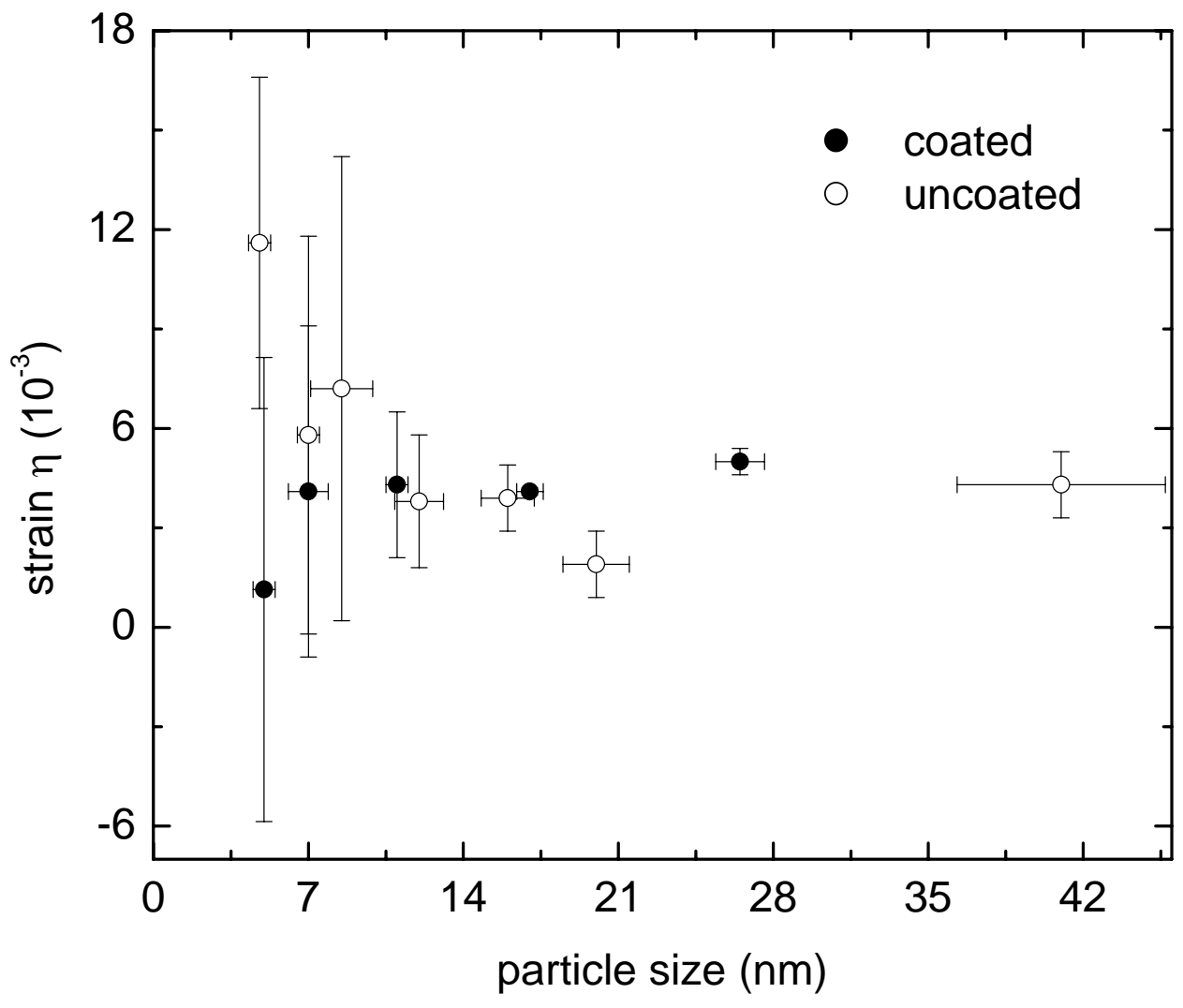

Fig. 3.9 Plot of the strain $\eta$ versus particle size for the coated and uncoated particles. 
In Table 3.1, we summarize the results of the particle sizes obtained from TEM and XRD analysis. For the smaller particles, the majority of the particles are in the form of nanorods, as shown in TEM pictures. For annealing temperature $T_{a} \geq 673 \mathrm{~K}$, nanorods change into particles with rounded morphology. This can be understood in terms of lowering of the surface energy with higher annealing temperatures. This also explains why the data in Fig.3.9 has high degree of scatter for smaller particles since the particles have a varied morphology and hence wider distribution of strain. From Table 3.1, it is evident that agreement between the sizes determined from XRD and TEM is generally good for the larger rounded particles. For the smaller particles with nanorod shapes, the calculated volume of the nanorods is quite close to the volume of a sphere of diameter D determined by XRD so that XRD measures average diameter of an equivalent sphere. Consequently, we have used the size D determined from XRD for labeling the figures. 


\begin{tabular}{|c|c|c|c|c|}
\hline & \multicolumn{2}{|c|}{ TEM (Rod) } & $\begin{array}{c}\text { TEM } \\
\text { (Sphere) }\end{array}$ & \multirow{2}{*}{$\begin{array}{c}\text { XRD (nm) } \\
\text { (uncoated) }\end{array}$} \\
\cline { 1 - 4 } Sample & $\begin{array}{c}\text { Diameter } \\
(\mathrm{nm})\end{array}$ & $\begin{array}{c}\text { Length } \\
(\mathrm{nm})\end{array}$ & $\begin{array}{c}\text { Diameter } \\
(\mathrm{nm})\end{array}$ & \\
\hline $\mathrm{NiO} 523 \mathrm{~K}$ & $1.7 \pm 0.5$ & $25 \pm 8$ & $5 \pm 1$ & $5 \pm 0.4$ \\
\hline $\mathrm{NiO} 573 \mathrm{~K}$ & $2.0 \pm 0.7$ & $20 \pm 5$ & $7 \pm 2$ & $7 \pm 1.4$ \\
\hline $\mathrm{NiO} 623 \mathrm{~K}$ & $3.0 \pm 0.8$ & $20 \pm 5$ & $9 \pm 2$ & $8 \pm 2.0$ \\
\hline $\mathrm{NiO} 673 \mathrm{~K}$ & - & - & $13 \pm 3$ & $12 \pm 1.1$ \\
\hline $\mathrm{NiO} 723 \mathrm{~K}$ & - & - & $16 \pm 3$ & $16 \pm 1.2$ \\
\hline $\mathrm{NiO} 773 \mathrm{~K}$ & - & - & $23 \pm 5$ & $20 \pm 1.5$ \\
\hline
\end{tabular}

Table 3.1 The summary of the particle size calculated from TEM analysis comparing the results derived by XRD measurements. 


\subsection{Structure Factor for NiO}

The scattering of $\mathrm{x}$-rays occurs due to the interaction of $\mathrm{x}$-ray photons with electrons in solids. Therefore there are two factors affecting the scattering of $\mathrm{x}$-ray beam: the atomic form factor $\mathrm{f}$ which depends on the density of electrons in the scatterer, and the geometrical structure factor $\mathrm{S}_{\mathrm{K}}$ whose form is determined by the basis of the scatterer. The phase difference between the incident and scattered beam is represented by exp $\{\mathrm{i}(\mathrm{k}-$ $\left.\left.\mathrm{k}^{\prime}\right) \cdot \mathrm{r}\right\}=\exp \{\mathrm{iK} \cdot \mathrm{r}\}$ where $\mathrm{K}$ is the reciprocal lattice vector and $\mathrm{r}$ is the position vector within the cell. In the case of a polyatomic crystal, the atomic form factor becomes $f_{j}$ for each atom $\mathrm{j}$ of the cell. Thus the structure factor has the form,

$$
\mathrm{S}_{\mathrm{K}}=\sum \mathrm{f}_{\mathrm{j}} \exp \left\{\mathrm{iK} \cdot \mathrm{r}_{\mathrm{j}}\right\}=\sum \mathrm{f}_{\mathrm{j}} \exp \left\{2 \pi \mathrm{i}\left(\mathrm{hx}_{\mathrm{j}}+\mathrm{ky}_{\mathrm{j}}+\mathrm{lz}_{\mathrm{j}}\right)\right\}
$$

For the NiO structure of sodium chloride, the basis consists of $\mathrm{Ni}^{2+}$ at $(000),\left(1 / 21 \frac{1}{2} 0\right),(0$ $1 / 21 / 2)$, and $(1 / 201 / 2)$ and $O^{2-}$ at $(1 / 21 / 21 / 2),\left(\begin{array}{lll}0 & 1 / 2\end{array}\right),(1 / 200)$, and $\left(\begin{array}{lll}0 & 1 / 2 & 0\end{array}\right)$. Let $f_{1}$ and $f_{2}$ be the atomic form factors of $\mathrm{O}^{2-}$ and $\mathrm{Ni}^{2+}$ ions respectively. Then Eq. (3.14) becomes,

$$
\begin{aligned}
\mathrm{S} & =4\left(\mathrm{f}_{\mathrm{Ni}}+\mathrm{f}_{\mathrm{O}}\right), & & \text { for hkl all even } \\
& =4\left(\mathrm{f}_{\mathrm{Ni}}-\mathrm{f}_{\mathrm{O}}\right), & & \text { for hkl all odd }
\end{aligned}
$$

Thus, for hkl all even, NiO has the lines (200), (220), (222), and (400), and the lines (111) and (311) for hkl all odd. Note that the scattered intensity is proportional to $|\mathrm{S}|^{2}$ so that all the observed lines in $\mathrm{NiO}$ have contributions from both the $\mathrm{Ni}$ and $\mathrm{O}$ atoms.

The position displacement of reflected lines can also occur due to the effect of temperature. The atoms in solids are vibrating as the temperature increases, leading to a change in their position. But the width of the diffracted line is not affected since the phonon frequency $\left(v \sim 10^{13} \mathrm{~Hz}\right)$ is negligible compared to the $\mathrm{x}$-ray frequency $\left(v \sim 10^{18}\right.$ $\mathrm{Hz}$ ). This temperature dependence of reflection lines is explained by the Debye-Waller Factor. If we assume that thermal vibrations are isotropic, for small displacement $\mathrm{u}_{\mathrm{j}}$, the structure facture of a cubic structure can be written as [Warren, 1996],

$$
\left[\mathrm{S}_{\mathrm{hkl}}\right]_{\mathrm{T}}=\mathrm{S}_{\mathrm{hkl}} \exp \left(-\mathrm{B} \sin ^{2} \theta / \lambda^{2}\right)
$$

where Debye - Waller temperature factor, $\left.B=8 \pi^{2} / 3<u_{j}^{2}\right\rangle$

or Intensity has the form, 


$$
\left[\mathrm{I}_{\mathrm{hk}}\right]_{\mathrm{T}}=\mathrm{I}_{0} \exp \left(-2 \mathrm{~B} \sin ^{2} \theta / \lambda^{2}\right)=\mathrm{m}(\mathrm{LP})|\mathrm{S}|^{2} \exp \left(-2 \mathrm{~B} \sin ^{2} \theta / \lambda^{2}\right)
$$

where $\mathrm{m}$ is the multiplicity factor, and Lorentz polarization factor (LP) $=\left\{\left(1+\cos ^{2}(2 \theta)\right) /\right.$ $\left.\sin ^{2} \theta \cos \theta\right\}$ [Warren, 1990].

We need to have a different $\mathrm{B}$ for $\mathrm{Ni}$ and $\mathrm{O}$ atoms from $\mathrm{Eq}$ (3.15). Thus, we let intensity $\mathrm{I}=\mathrm{m}(\mathrm{LP})\left\{\mathrm{f}_{\mathrm{Ni}} \exp \left(-\mathrm{B}_{\mathrm{Ni}} \sin ^{2} \theta / \lambda^{2}\right)+\mathrm{f}_{\mathrm{O}} \exp \left(-\mathrm{B}_{\mathrm{O}} \sin ^{2} \theta / \lambda^{2}\right)\right\}^{2}$ for hkl all even and $I=m(L P)\left\{f_{N i} \exp \left(-B_{N i} \sin ^{2} \theta / \lambda^{2}\right)-f_{O} \exp \left(-B_{O} \sin ^{2} \theta / \lambda^{2}\right)\right\}^{2}$ for hkl all odd. To reduce the symbols, let $\mathrm{Q}_{\text {even }}=(\mathrm{I} / \mathrm{m}(\mathrm{LP}))^{1 / 2}{ }_{\text {even }}$ and $\mathrm{Q}_{\text {odd }}=(\mathrm{I} / \mathrm{m}(\mathrm{LP}))^{1 / 2}{ }_{\text {odd }}$ for each case. Now we can determine $\mathrm{B}_{\mathrm{Ni}}$ and $\mathrm{B}_{\mathrm{O}}$ by combining these relations,

$$
\begin{aligned}
& \ln \left\{\left(\mathrm{Q}_{\text {even }}+\mathrm{Q}_{\text {odd }}\right) / 2 \mathrm{f}_{\mathrm{Ni}}\right\}=-\mathrm{B}_{\mathrm{Ni}}\left(\sin ^{2} \theta / \lambda^{2}\right) \\
& \ln \left\{\left(\mathrm{Q}_{\text {even }}-\mathrm{Q}_{\text {odd }}\right) / 2 \mathrm{f}_{\mathrm{O}}\right\}=-\mathrm{B}_{\mathrm{O}}\left(\sin ^{2} \theta / \lambda^{2}\right)
\end{aligned}
$$

Here, an example of how to calculate the $\mathrm{B}_{\mathrm{Ni}}$ and $\mathrm{B}_{\mathrm{O}}$ for $\mathrm{NiO} 5 \mathrm{~nm}$ uncoated sample is given. Table 3.2 shows the calculation of each line from the $\mathrm{x}$-ray data and the atomic scattering factors $\mathrm{f}_{\mathrm{Ni}}$ and $\mathrm{f}_{\mathrm{O}}$ which depend on the angle $\theta$. Using this data, we draw the graph $\mathrm{Q}=(\mathrm{I} / \mathrm{mLP})^{1 / 2}$ vs. $\sin \theta / \lambda$ for hkl all even and odd each.. In Fig. 3.10, the linear equation represents the $\mathrm{Q}_{\text {even }}$ and $\mathrm{Q}_{\text {odd }}$ for each $\sin \theta / \lambda$. This calculation is shown in Table 3.3. Now we let $\left(\mathrm{Q}_{\text {even }}+\mathrm{Q}_{\text {odd }}\right) / 2 \mathrm{f}_{\mathrm{Ni}}$ be $\mathrm{Q}^{+}$and $\left(\mathrm{Q}_{\text {even }}-\mathrm{Q}_{\text {odd }}\right) / 2 \mathrm{f}_{\mathrm{O}}$ be $\mathrm{Q}^{-}$and finally we can get the $\mathrm{B}_{\mathrm{Ni}}$ and $\mathrm{B}_{\mathrm{O}}$ from the slopes of the relation $\ln \left(\mathrm{Q}^{+}\right)$and $\ln \left(\mathrm{Q}^{-}\right)$respectively which is shown in Fig. 3.11.

We summarize the Debye-Waller Temperature Factor for all uncoated particles in Table 3.4 and make a graph of the results in Fig. 3.12. Through the graph, we know that the thermal vibrations of nickel increase for small particle size less than $12 \mathrm{~nm}$ and in case of oxygen, $\mathrm{B}_{\mathrm{O}}$ decreases as particle size decreases. 


\begin{tabular}{|c|c|c|c|c|c|c|c|c|}
\hline \hline $\mathrm{hkl}$ & $\theta$ & $\mathrm{m}$ & $\mathrm{I}$ & $\mathrm{LP}$ & $(\mathrm{I} / \mathrm{mLP})^{1 / 2}$ & $\mathrm{f}_{\mathrm{Ni}}$ & $\mathrm{f}_{\mathrm{O}}$ & $\sin \theta / \lambda$ \\
\hline$(111)$ & 18.56 & 8 & 305 & 15.74 & 1.556 & 18.55 & 4.83 & 0.2065 \\
\hline$(200)$ & 21.61 & 6 & 601 & 11.32 & 2.975 & 17.55 & 4.46 & 0.2389 \\
\hline$(220)$ & 31.25 & 12 & 374 & 5.09 & 2.474 & 14.73 & 3.34 & 0.3365 \\
\hline$(311)$ & 37.50 & 24 & 113 & 3.58 & 1.146 & 13.32 & 2.78 & 0.3948 \\
\hline$(222)$ & 39.40 & 8 & 81 & 3.31 & 1.749 & 12.88 & 2.53 & 0.4117 \\
\hline$(400)$ & 47.40 & 6 & 46 & 2.74 & 1.672 & 11.87 & 2.16 & 0.4774 \\
\hline \hline
\end{tabular}

Table 3.2 shows the information about each Bragg peak in the x-ray pattern of $5 \mathrm{~nm}$ uncoated $\mathrm{NiO}$. 


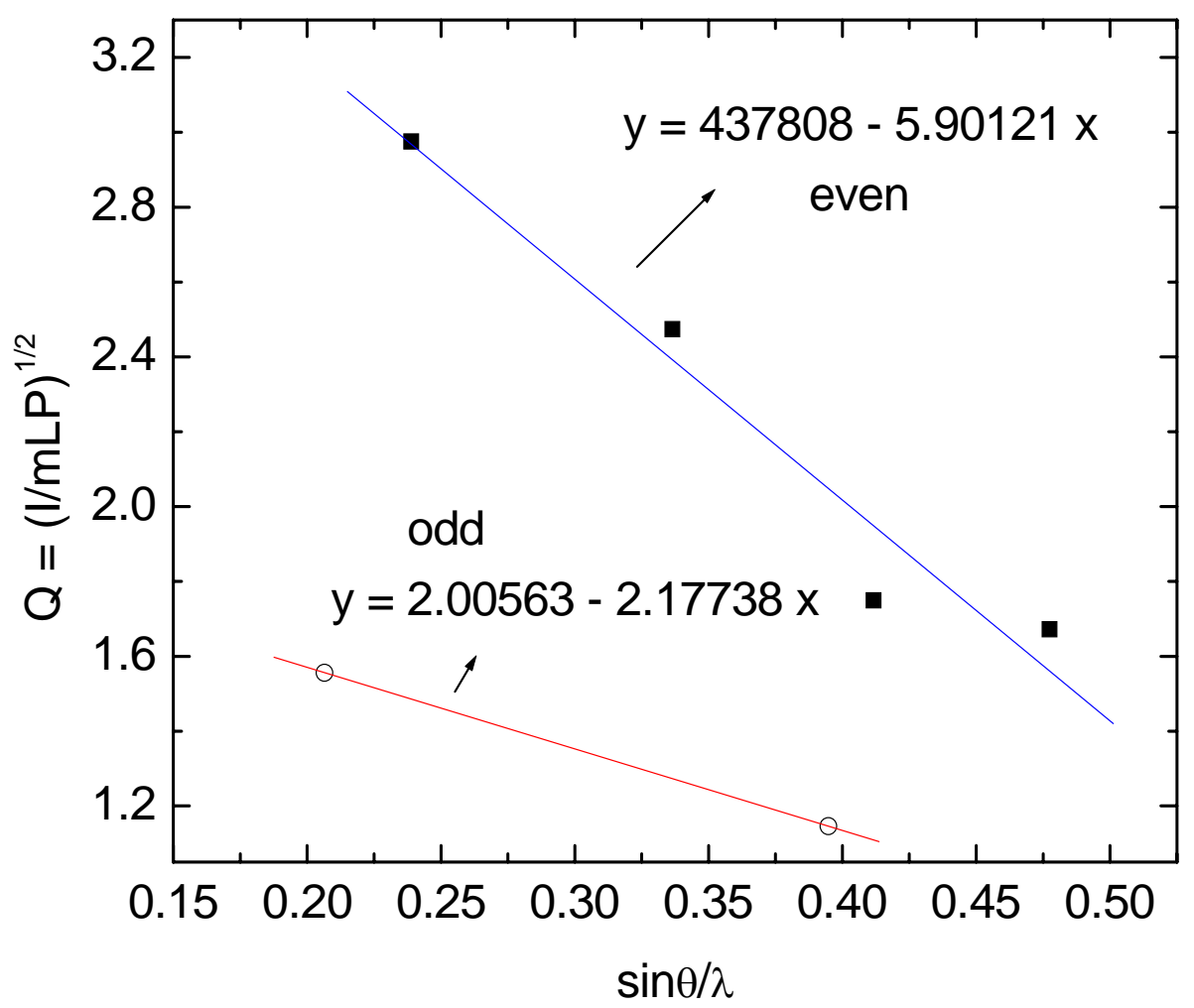

Fig. 3.10 Plot of $\mathrm{Q}=(\mathrm{I} / \mathrm{mLP})^{1 / 2}$ vs. $\sin \theta / \lambda$ for hkl all even and odd. 


\begin{tabular}{|c|c|c|c|c|c|c|}
\hline \hline$(\sin \theta / \lambda)^{1 / 2}$ & $Q_{\text {odd }}$ & $\mathrm{Q}_{\text {even }}$ & $\mathrm{Q}^{+}$ & $\mathrm{Q}^{-}$ & $\ln \left(\mathrm{Q}^{+}\right)$ & $\ln \left(\mathrm{Q}^{-}\right)$ \\
\hline 0.0426 & 1.556 & 3.16 & 0.1271 & 0.0432 & -2.0628 & -3.1419 \\
\hline 0.0571 & 1.485 & 2.987 & 0.1269 & 0.0423 & -2.0644 & -3.1630 \\
\hline 0.1132 & 1.273 & 2.392 & 0.1244 & 0.0380 & -2.0843 & -3.2702 \\
\hline 0.1559 & 1.146 & 2.048 & 0.1199 & 0.0339 & -2.1211 & -3.3843 \\
\hline 0.1695 & 1.109 & 1.949 & 0.1187 & 0.0326 & -2.1312 & -3.4234 \\
\hline 0.2279 & 0.966 & 1.561 & 0.1064 & 0.0251 & -2.2405 & -3.6849 \\
\hline \hline
\end{tabular}

Table 3.3 summarizes the results of the calculation from Fig 3.10. 


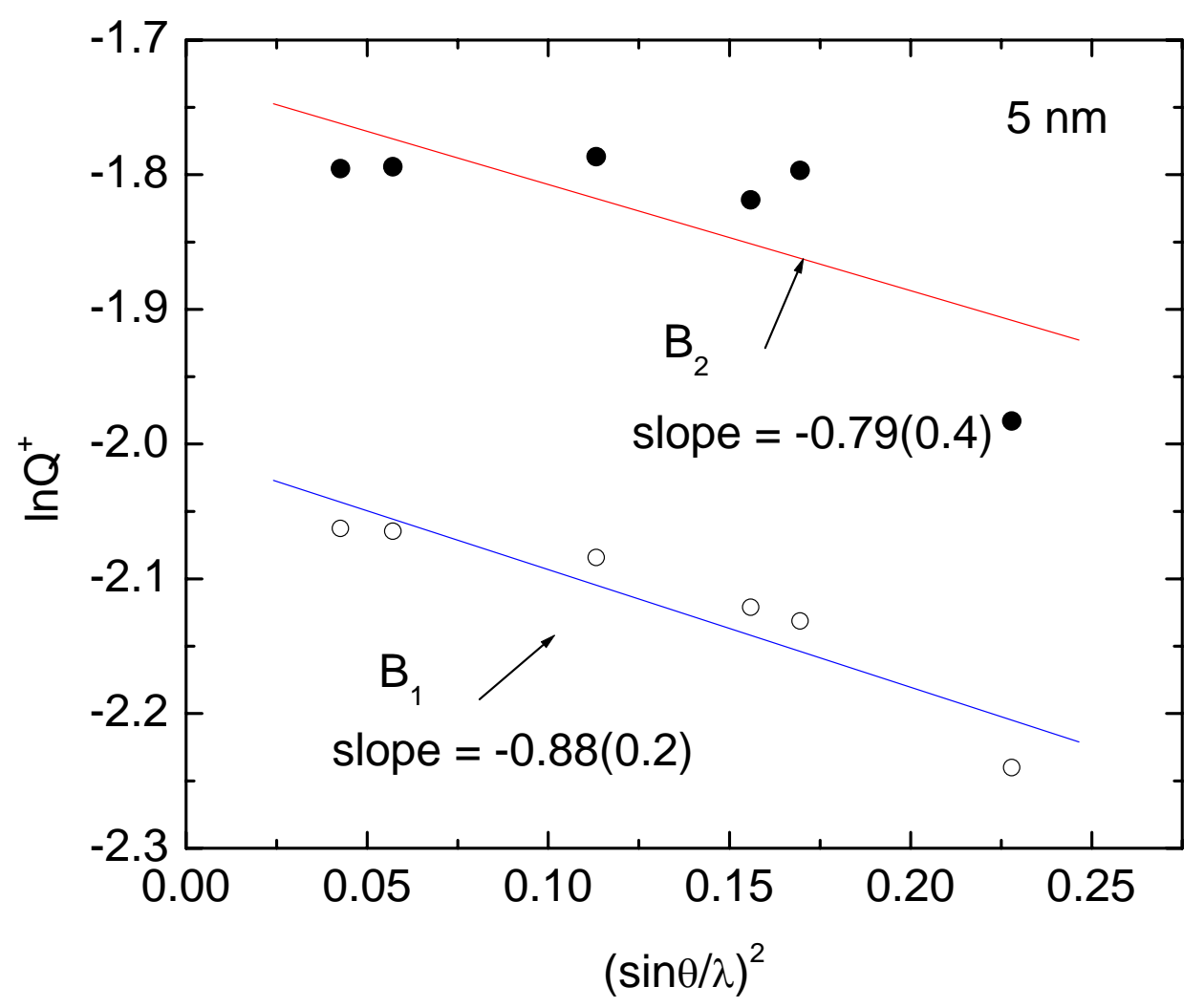

Fig. 3.11 Plots of $\ln \mathrm{Q}^{+}\left(\ln \mathrm{Q}^{-}\right)$against $(\operatorname{Sin} \theta / \lambda)^{2}$, the slopes give directly the values of $\mathrm{B}_{\mathrm{Ni}}$ $(\mathrm{Ni})$ and $\mathrm{B}_{\mathrm{O}}(\mathrm{O})$. 


\begin{tabular}{|c|c|c|}
\hline \hline Particle size $(\mathrm{nm})$ & $\mathrm{B}_{\mathrm{Ni}}$ & $\mathrm{B}_{\mathrm{O}}$ \\
\hline 5 & $0.88(0.18)$ & $0.79(0.36)$ \\
\hline 7 & $1.24(0.19)$ & $0.81(0.31)$ \\
\hline 8 & $0.69(0.17)$ & $0.68(0.38)$ \\
\hline 12 & $0.73(0.19)$ & $1.39(0.39)$ \\
\hline 16 & $0.10(0.18)$ & $0.80(0.33)$ \\
\hline 20 & $0.48(0.18)$ & $0.97(0.34)$ \\
\hline 41 & $0.33(0.19)$ & $1.32(0.40)$ \\
\hline bulk & $0.12(0.18)$ & $0.53(0.32)$ \\
\hline \hline
\end{tabular}

Table 3.4 shows the Debye - Waller Temperature Factor for all particle sizes (uncoated).

The Deby-Waller factor takes into account the effect of lattice vibrations on the diffracted-beam amplitudes. From Table 3.4, it is noted that there is some particle size dependence of the Debye-Waller factors. For smaller size samples below $10 \mathrm{~nm}$, the Debye-Waller factors of nickel and oxide becomes larger than the values of the bulk $\mathrm{NiO}$ although there is considerable experimental uncertainty due to scatter of the data. As particle size increases, the value for nickel tends to decrease close to the bulk value whereas the value of oxygen tends to increase. There are several theoretical models proposed for sodium chloride structure. One of those models, a so-called shell model (SM) was proposed [Dick et al, 1958], in which the atom is represented by a core consisting of the nucleus and inner electrons and a shell with the outer electrons. In this $\mathrm{SM}$ model at $\mathrm{T}=293 \mathrm{~K}$, the calculated Debye-Waller factors are $\mathrm{B}_{\mathrm{Ni}}=0.2624$ for $\mathrm{Ni}$ and $\mathrm{B}_{\mathrm{O}}=0.3567$ for $\mathrm{O}$. These values are comparable with the result of our bulk sample. 


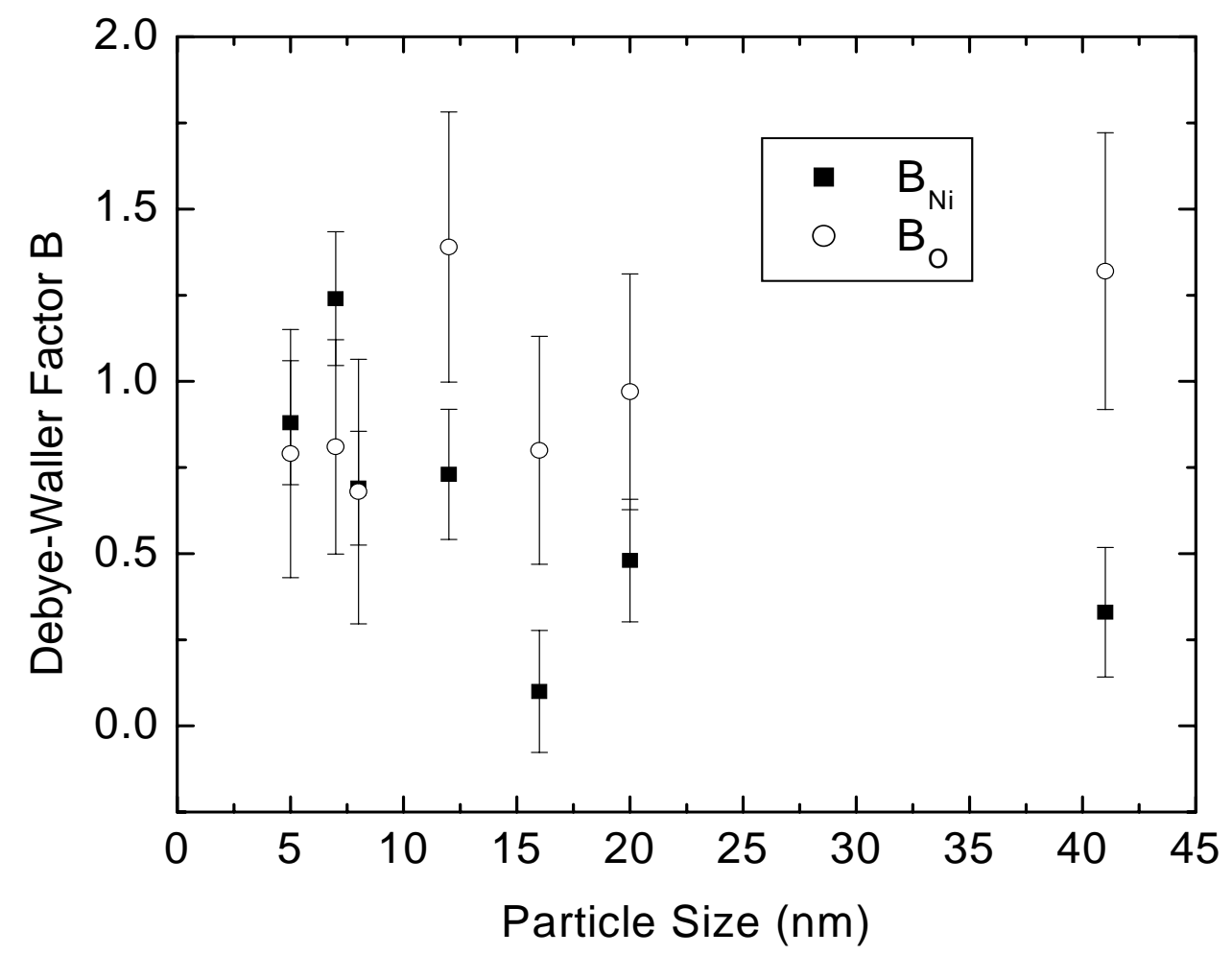

Fig. 3.12 Plots of Debye-Waller factor vs. D using the results from Table 3.4. 


\section{CHAPTER IV}

\section{Review of Superparamagnetism}

\subsection{Superparamagnetic Particles}

Néel's pioneering work for the magnetic properties of single-domain particles was followed by many investigations of these nanoparticles because these nano-sized magnetic particles have applications in technology such as, magnetic recording media, ferrrofluids, magnetic refrigeration, catalysts, and soft magnetic materials [Dormann et al, 1992 and references in]. However, the magnetic properties are not easily understood because there are complex things to take into account such as particle size distributions, magnetic interparticle interactions, and magnetic anisotropy. In this chapter, I present a review of the basic properties of the magnetic nanoparticles, based in part on the material in the book by Morrish [Morrish, 2001]. This review will be important for discussing the magnetic properties of $\mathrm{NiO}$ nanoparticles presented in Chapters $\mathrm{V}$ and VI.

When particles have a magnetization which changes spontaneously due to the thermal agitation, similar to the paramagnetic atoms, with the exception that magnetic moment is very large, these particles are said to exhibit superparamagnetism, first introduced by Néel [Néel, 1962]. This phenomenon is investigated in single-domain size with uniaxial anisotropy which has an easy direction along the z-axis. Here, we assume that the particle's magnetization $(\mathrm{M})$ remains constant for the applied field to simplify the analysis.

Consider a prolate ellipsoidal particle in a magnetic field as shown in Fig. 4.1. The applied field makes magnetization, $M$, to lie in the plane at the angles of, $\alpha, \varphi$ respect to the polar axis and the field respectively. The shape anisotropy produces demagnetization energy per unit volume given by

$$
\mathrm{F}_{\mathrm{D}}=1 / 2\left\{(\mathrm{M} \cos \alpha)^{2} \mathrm{D}_{\mathrm{a}}+(\mathrm{M} \sin \alpha)^{2} \mathrm{D}_{\mathrm{b}}\right\}
$$

where $D_{a}$ and $D_{b}$ are the demagnetization coefficients along the polar axis a and the equatorial axis $b$ direction. For simplicity, we assume that the exchange energy $F_{e x}=0$. 


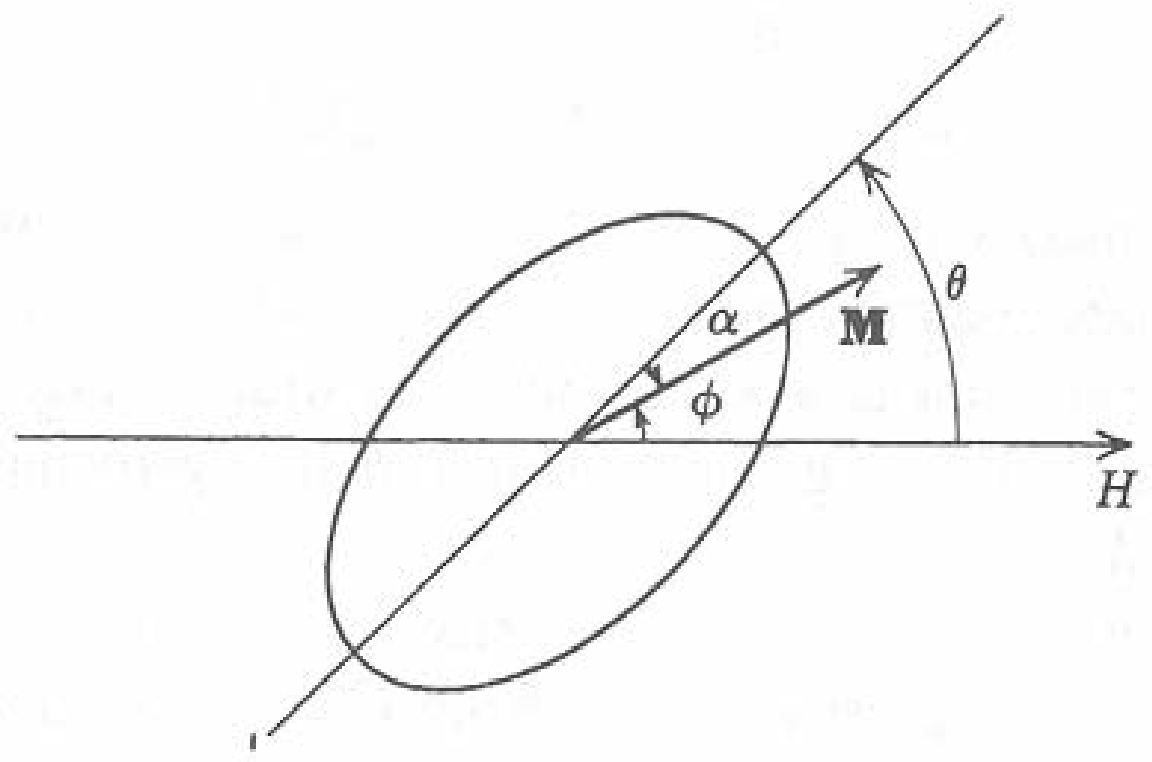

Fig. 4.1 This diagram shows the prolate ellipsoidal particle with an applied magnetic field H. 
In Chapter II, we treated a $\mathbf{S} \cdot \mathbf{S}$ term of the Heisenberg exchange interaction energy as a scalar product, assuming coordinate symmetry. In reality, there is a preferred direction for the magnetization due to the lack of the symmetry. This effect is called, crystalline anisotropy. For an ellipsoidal particle, the demagnetization energy acts as anisotropy energy. This anisotropy energy is a minimum if $\mathrm{M}$ lies along the polar axis, called the easy direction whereas if $\mathrm{M}$ lies along an equatorial axis, called the hard direction, the energy is a maximum. Eq. (4.1) can be modified into the form,

$$
F_{D}=1 / 2 M^{2} D_{a}+1 / 2 M^{2}\left(D_{b}-D_{a}\right) \sin ^{2} \alpha .
$$

So, for a particle with volume $\mathrm{V}$, the variable part of the energy in the above equation is

$$
\begin{aligned}
F_{T} & =1 / 2 M^{2}\left(D_{b}-D_{a}\right) V \sin ^{2} \alpha \\
& =1 / 2 C V \sin ^{2} \alpha
\end{aligned}
$$

where $C=M^{2}\left(D_{b}-D_{a}\right)$, for $C=2 K$, where $K$ here corresponds to the magnetocrystalline anisotropy constant. Fig. 4.2 shows the energy $\mathrm{F}_{\mathrm{T}}$ vs. $\alpha$ in Eq. (4.3). There are two minimum energy states at $\alpha=0$ and $\pi$ with the opposite directions and these states are separated by an energy barrier of height $1 / 2 \mathrm{CV}$ at $\alpha=\pi / 2$. This means that to switch the state from the parallel direction, $\alpha=0$, to the antiparallel direction, $\alpha=\pi$, we have to overcome the energy barrier of $1 / 2 \mathrm{CV}$. Thus, the magnetization is stable if there are no perturbations such as a thermal agitation. For small particles, as the energy barrier is lowered due to the small volume, thermal energy is sufficient to change the magnetization between $\alpha=0$ and $\alpha=\pi$ making the average of the remanence $<\mathrm{M}_{\mathrm{r}}>$ equal to zero. The magnetization flips up or down states due to thermal energy as in a paramagnetic particle but here the magnetic moment is very large. This phenomenon is called superparamagnetism.

One important characteristic of superparamagnetism is the superparamagnetic relaxation time $\tau$ which is the average time the magnetization spends in the minima of the anisotropy energy. Now, consider an assembly of particles with a uniaxial anisotropy along $\mathrm{z}$-axis. In a large field $\mathrm{H}$ along the $\mathrm{z}$ direction, all the particles are magnetized to saturation M. And if the field is removed, magnetization will decay due to thermal agitation according to the relation,

$$
\mathrm{M}_{\mathrm{H}}=\mathrm{M} \exp (-\mathrm{t} / \tau)
$$


If $\tau$ is very large, and $M_{H}=M$, the system is stable. The relaxation rate $1 / \tau$ must be proportional to the Boltzmann factor $\exp \left(-\Delta \mathrm{E} / \mathrm{k}_{\mathrm{B}} \mathrm{T}\right)$ since $\Delta \mathrm{E}$ is the energy barrier $(1 / 2 \mathrm{CV})$ between the two energy minima. Thus, we can write the relaxation rate as,

$$
1 / \tau=1 / \tau_{0} \exp \left(-\Delta \mathrm{E} / \mathrm{k}_{\mathrm{B}} \mathrm{T}\right)=\mathrm{f}_{0} \exp \left(-\mathrm{CV} / 2 \mathrm{k}_{\mathrm{B}} \mathrm{T}\right)
$$

where $\mathrm{k}_{\mathrm{B}}$ is Boltzmann's constant and $\mathrm{f}_{0}$ is the attempt frequency typically in the range $10^{9}$ to $10^{12}$ sec. According to Eq. (4.5), $\mathrm{f}=1 / \tau$ is a strong function of temperature $\mathrm{T}$ and $\mathrm{f}$ decreases as $\mathrm{T}$ decreases. At a certain temperature $\mathrm{T}_{\mathrm{B}}$, the relaxation rate $\mathrm{f}$ will become equal to $f_{m}$, the frequency of measurement. This leads to

or

$$
\begin{aligned}
& \mathrm{f}_{\mathrm{m}}=\mathrm{f}_{0} \exp \left(-\Delta \mathrm{E} / \mathrm{k}_{\mathrm{B}} \mathrm{T}_{\mathrm{B}}\right) \\
& \mathrm{T}_{\mathrm{B}}=\left(\Delta \mathrm{E} / \mathrm{k}_{\mathrm{B}}\right) / \ln \left(\mathrm{f}_{0} / \mathrm{f}_{\mathrm{m}}\right) .
\end{aligned}
$$

According to Eq. (4.7), $T_{B}$ depends on the frequency of measurement, $f_{m}$. The higher the $f_{m}$, the higher the $T_{B}$. For magnetization measurements, if we assume that the experimental measuring time $\tau=1 / \mathrm{f}_{\mathrm{m}} \approx 10^{2} \mathrm{sec}, \mathrm{f}_{0} \approx 10^{9}$, Eq. (4.7) will have the relation,

$$
\Delta \mathrm{E}=1 / 2 \mathrm{CV}=25 \mathrm{k}_{\mathrm{B}} \mathrm{T}_{\mathrm{B}} \text {. }
$$

For each particle with size $\mathrm{V}$, there is a corresponding temperature $\mathrm{T}_{\mathrm{B}}$, above which the particles behave like a superparamagnet. I will use the above equations to interpret our results in Chapter V.

The existence of this superparamagnetism (SP) can be tested experimentally. No remanence in the experiment of $M$ vs. $H$ is observed for $S P$ for $T>T_{B}$. SP can be destroyed by cooling as the relaxation time varies exponentially with temperature. The temperature below which the system is stable or blocked is called the blocking temperature $\mathrm{T}_{\mathrm{B}}$. Therefore, through measuring the remanence, we can see the increase of remanence proportional to the amount of the particles which are superparamagnetic as temperature decreases. In another experiment, magnetization $\mathrm{M}$ vs. $\mathrm{H}$ at different temperatures above blocking temperature is measured. If the particle is superparamagnetic, the experimental results have to follow the Langevin function which is valid for single domain superparamagnetic nanoparticles. This means that the plots of normalized magnetization vs. H/T measured at various temperatures should collapse into one single graph. Here, we obtain very large magnetic moments $\mu_{\mathrm{P}}$ estimated from the Langevin function, 


$$
\mathrm{M}_{\mathrm{H}}(\mathrm{T})=\mathrm{M}(0) \mathrm{L}(\mathrm{x})=\mathrm{M}(0)\{\operatorname{coth}(\mathrm{x})-1 /(\mathrm{x})\}
$$

where $\mathrm{x}$ is $\mu_{\mathrm{P}} \mathrm{H} / \mathrm{k}_{\mathrm{B}} \mathrm{T}$. In the limit of very small $\mathrm{x}, \mathrm{L}(\mathrm{x}) \approx \mathrm{x} / 3$ and then the magnetization is

$$
\mathrm{M}_{\mathrm{H}}(\mathrm{T})=\mathrm{M}(0) \mu_{\mathrm{P}} \mathrm{H} / 3 \mathrm{k}_{\mathrm{B}} \mathrm{T}=\mathrm{C} / \mathrm{T}
$$

Eq (4.10) is the Curie law for paramagnetism, with the Curie constant $C=M(0) \times \mu_{\mathrm{P}} / 3 \mathrm{k}_{\mathrm{B}}$ for superparamagnetism. 


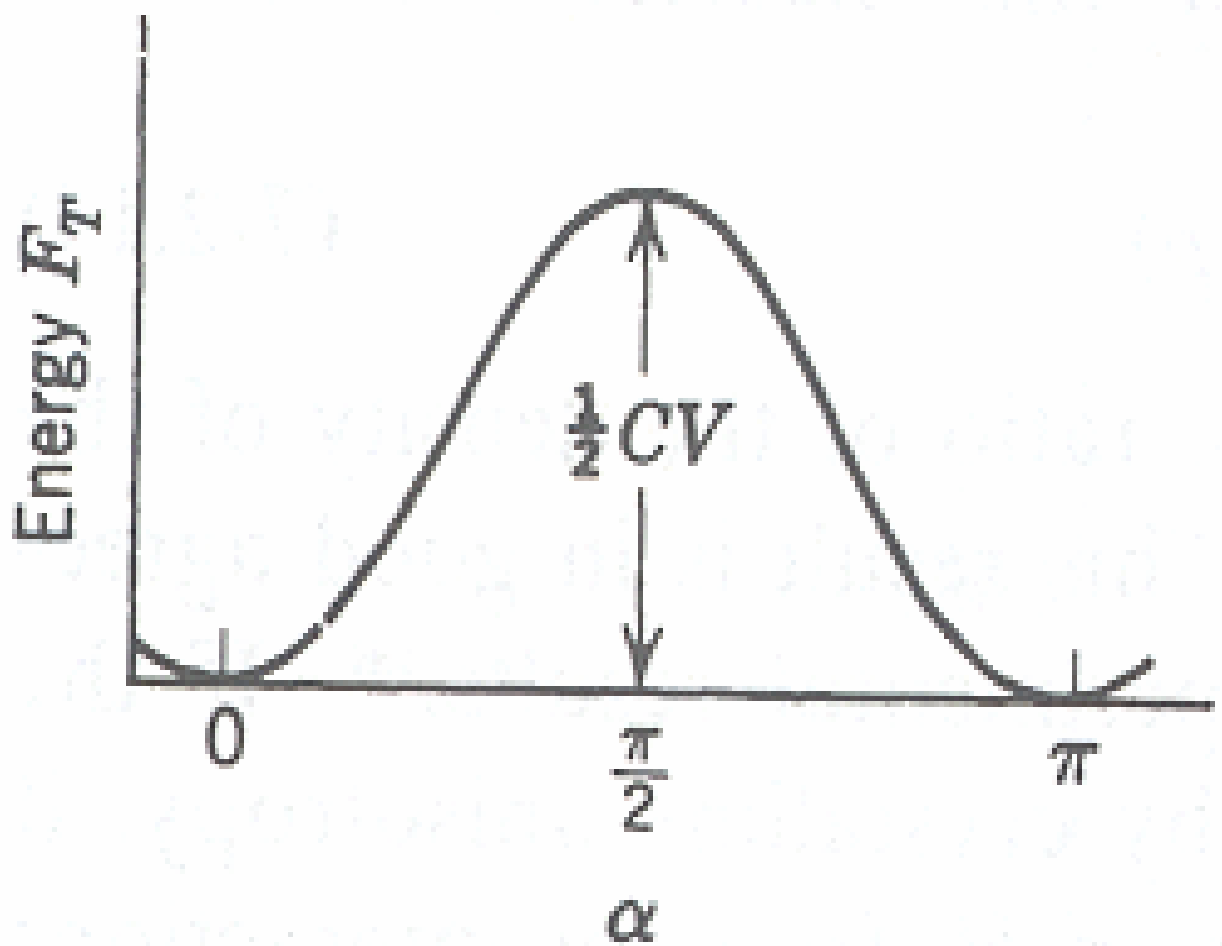

Fig. 4.2 For a single particle, the energy with an angle, $\alpha$ is separated by the energy barrier $1 / 2 \mathrm{CV}$ at $\alpha=\pi / 2$ between the two equivalent state at $\alpha=0$ and $\alpha=\pi$ [Morrish, 2001]. 


\subsection{Critical size for single domain particles in ferromagnets}

A ferromagnetic material, as described in Chapter II, has a magnetic moment, even in the absence of a magnetic field. This is explained by a spontaneous magnetization. In spite of this property, a ferromagnetic material may exhibit no magnetic moment without applying a field. These results can be explained using the concept of small region, called domain postulated by Weiss [1907]. Generally, ferromagnetic particles consist of many domains all oriented in different directions in $\mathrm{H}=0$ so that the total magnetization is zero. If the particle size is small or a single domain, then for such particles, the magnetization is large even in $\mathrm{H}=0$, giving rise to superparamagnetism. Thus, when we treat the SP-NP, it is important to know the critical size for a single-domain in zero applied fields. To estimate the critical size, we need to consider a domain wall, often called Bloch wall (Bloch, 1932) which separates two domains in a bulk ferromagnet.

A Bloch wall in a ferromagnet is a transition region which separates adjacent domains having different directions of magnetization. Exchange energy of this region has the form,

$$
\mathrm{F}_{\mathrm{EX}}=<\mathrm{H}_{\mathrm{EX}}>=-2 \mathrm{~J}_{\mathrm{e}} \mathrm{S}^{2} \cos \varphi
$$

where $\varphi$ is the angle between neighboring spins. For small $\varphi$, we can let $\cos \varphi$ be (1 $\left.\varphi^{2} / 2\right)$ and hence Eq. (4.11) is divided into constant part and $\varphi$ dependant part which need to be considered. In a Bloch wall, the total change in angle is $\pi$ since the magnetization is reversed between up and down sides. If the total change of $\pi$ occurs in $\mathrm{N}$ steps, the angle $\varphi$ between spins is $\pi / N$. Thus, the variable part of the exchange energy is $\mathrm{J}_{\mathrm{e}} \mathrm{S}^{2} \pi^{2} / \mathrm{N}$ for $\mathrm{N}$ atoms. If all spins are parallel to the easy axis, the anisotropy energy, $F_{K}$ will be minimized. However, because of the domain wall, $\mathrm{F}_{\mathrm{K}}$ is not in the minimum and so the thickness of the wall is limited by this crystalline energy. If we let "a" be the lattice constant, then the wall thickness $\delta$ is $\mathrm{Na}$. Therefore the wall energy $\mathrm{F}_{\mathrm{WALL}}$ per unit area of the wall is

$$
\mathrm{F}_{\mathrm{WALL}}=\mathrm{F}_{\mathrm{EX}}+\mathrm{F}_{\mathrm{K}}=\mathrm{J}_{\mathrm{e}} \mathrm{S}^{2} \pi^{2} / \mathrm{a} \delta+\mathrm{K} \delta .
$$

By minimizing this energy with respect to $\delta$, we obtain the wall thickness $\delta=\left(\mathrm{J}_{\mathrm{e}} \mathrm{S}^{2} \pi^{2}\right.$ $/ \mathrm{Ka})^{1 / 2}$. We substitute $\delta$ into Eq. (4.12) leading to minimum wall energy as

$$
\mathrm{F}_{\mathrm{WALL}}=2 \pi \mathrm{S}\left(\mathrm{J}_{\mathrm{e}} \mathrm{K} / \mathrm{a}\right)^{1 / 2}
$$


Now we calculate the critical size below which a ferromagnetic material will exist as a single domain particle. For a spherical particle with diameter $d$, the magnetic energy of the uniform state is $\mathrm{M}_{\mathrm{S}}{ }^{2} \mathrm{~d}^{3}$ for the volume $\mathrm{d}^{3}$, and there exist Bloch wall energy separating the domains. Thus, the critical size is calculated by letting the magnetic energy for the volume equal to wall energy for area,

$$
\mathrm{M}_{\mathrm{S}}^{2} \mathrm{~d}_{\mathrm{C}}^{3}=\mathrm{F}_{\mathrm{WALL}} \mathrm{d}_{\mathrm{C}}^{2}
$$

So, we obtain the critical size for single domain particles in ferromagnets,

$$
\mathrm{d}_{\mathrm{C}}(\mathrm{cm})=\mathrm{F}_{\mathrm{WALL}} / \mathrm{M}_{\mathrm{S}}{ }^{2}=1 / \mathrm{M}_{\mathrm{S}}{ }^{2} \times\left\{2 \pi \mathrm{S}\left(\mathrm{J}_{\mathrm{e}} \mathrm{K} / \mathrm{a}\right)^{1 / 2}\right\}
$$

Note if $\mathrm{M}_{\mathrm{S}}$ is small, $\mathrm{d}_{\mathrm{C}}$ is large. That is why usually ferrimagnets are used for applications. For example, if $\mathrm{M}_{\mathrm{S}} \approx 1700 \mathrm{Oe}$ and $\mathrm{F}_{\mathrm{W}}=3 \mathrm{ergs} / \mathrm{cm}^{2}$ for Fe, then from Eq. (4.15), $\mathrm{d}_{\mathrm{C}} \approx 10^{-6}$ $\mathrm{cm} \approx 100 \AA$. That is particles with size less than $\mathrm{d}_{\mathrm{C}} \approx 100 \AA$ will be single domain.

\subsection{Superparamagnetism in Antiferromagnetic Nanoparticles}

Néel described the magnetic structure of antiferromagnetic (AF) nanoparticles as an exchange-coupled spherical bilayer composed of an inner antiferromagnetic core and an outer shell of uncompensated spins [Néel et al, 1962]. As a particle size becomes smaller, the percentage of surface spins becomes much larger compared to that in bulk materials. Thus, for small particles, the effect of the surface spins is significant. The spins in the interior are governed by the normal exchange and anisotropy terms. However, the surface spins have only exchange interactions with interior spins. The exchange interaction between these two layers creates a unidirectional anisotropy which may cause

superparamagnetism due to uncompensated spins. This can produce a ferromagnetic/antiferromagnetic (F/AF) interface, leading to a shift of hysteresis loop, the so-called exchange bias phenomenon [Berkowitz et al, 1999 and Nogues et al, 1999]. Therefore, nanoparticles of AF material have important applications in devices which use the exchange bias, such as spin valves. 
For NiO, Néel considered a two-sublattice model with A and B as the sublattices. If the number of atoms, $\mathrm{n}_{\mathrm{A}}$ and $\mathrm{n}_{\mathrm{B}}$, are equal, the usual antiferromagnetic state follows. But if not, a net magnetic moment is proportional to $p=n_{A}-n_{B}$. Néel considered three cases as shown in Fig. 4.3, with details given in later papers [Richardson et al, 1991 and Mangin et al, 1999]. In case I, atoms are randomly ordered and the probability to find atom $\mathrm{A}$ without $\mathrm{B}$ is proportional to the square root of the number of atoms leading $\mathrm{p}=$ $(n)^{1 / 2}$. In case II, ordered pairs are occupied on complete sublattices. For case IIa and IIb, they are completely compensated in their ordering resulting in $p$ being zero. For case IIc, the atoms on the top and bottom layers have the same orientation and this case yields $p=$ $(n)^{2 / 3}$. And in last case III, the pairs have incomplete ordered sublattices with $p=(n)^{1 / 3}$. For each case, the magnetic moment $\mu_{\mathrm{P}}$ is represented by $\mu_{\mathrm{P}}=\mathrm{p} \mu_{\mathrm{A}} \mu_{\mathrm{B}}$ where $\mu_{\mathrm{A}}$ is the atomic spin moment and $\mu_{\mathrm{B}}$ is the Bohr magneton.

In this work, we show that the above 2-sublattice model cannot explain our experimental observations. Kodama et al. [Kodama et al, 1997] proposed that the multisublattice ordering may be necessary to explain the large magnetic moment and large coercivities observed in $\mathrm{NiO}$ nanoparticles. Their calculation predicted the nature of ordering as the particle size is changed.

For AF materials, the Langevin function in Eq. (4.9) has to be modified to

$$
\mathrm{M}=\mathrm{M}_{0} \mathrm{~L}\left(\mu_{\mathrm{P}} \mathrm{H} / \mathrm{k}_{\mathrm{B}} \mathrm{T}\right)+\chi_{\mathrm{a}} \mathrm{H}
$$

where $\mathrm{M}_{0}$ is the saturation magnetization and $\chi_{\mathrm{a}}$ is the susceptibility of the antiferrmmagnetically ordered nanoparticle core. This modified Langevin function is used to determine $\mu_{\mathrm{P}}$ by fitting $\left(\mathrm{M}-\chi_{\mathrm{a}} \mathrm{H}\right) / \mathrm{M}_{0}$ against $\mathrm{H} / \mathrm{T}$ for temperatures between the blocking temperature $T_{B}$ and the Néel temperature $T_{N}$. 


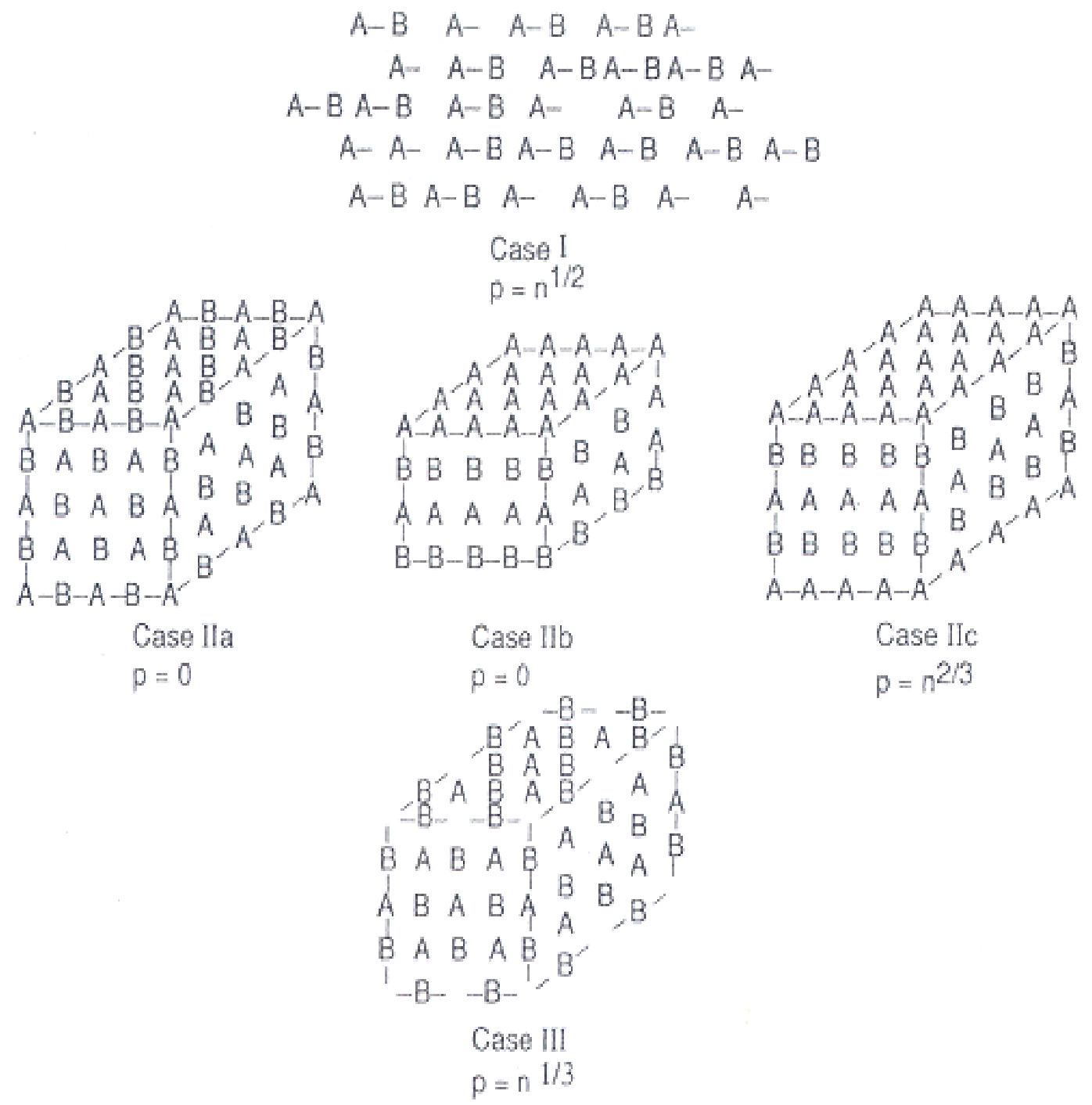

Fig. 4.3 shows the origin of particle magnetic moments for antiferromagnetic $\mathrm{NiO}$ particles proposed by Néel. 


\subsection{The behavior of M vs. H (Stoner-Wohlfarth Model)}

Here, I summarize how the magnetization of a single-domain particle will respond to applied field. Consider the same case for single domain particle as shown in Fig. 4.1. If we take into account the applied field part in Eq. (4.3), the total energy will be,

$$
\mathrm{F}_{\mathrm{T}}=\text { const }-1 / 4 \mathrm{M}^{2}\left(\mathrm{D}_{\mathrm{b}}-\mathrm{D}_{\mathrm{a}}\right) \mathrm{V} \cos 2 \alpha-\mathrm{HMV} \cos \varphi
$$

where $\varphi$ is the angle between $\mathrm{M}$ and $\mathrm{H}$. To find the equilibrium state, we minimize $\mathrm{F}_{\mathrm{T}}$ with respect to $\varphi$ and obtain

$$
\partial \mathrm{F}_{\mathrm{T}} / \partial \varphi=1 / 2 \mathrm{M}^{2}\left(\mathrm{D}_{\mathrm{b}}-\mathrm{D}_{\mathrm{a}}\right) \mathrm{V} \sin 2 \alpha+\mathrm{HM} \sin \varphi=0 .
$$

This equation satisfies a minimum with condition of $\left(\partial \mathrm{F}_{\mathrm{T}} / \partial \varphi\right)^{2}>0$. Since $\mathrm{M} \times \mathrm{H}=\mathrm{MH}$ $\sin \varphi$ is the torque acting on the magnetization due to applied field, Eq. (4.18) means that when the torque by demagnetization and applied field are the same, the system is stable. Eq. (4.18) can be represented as this,

$$
1 / 2 \sin 2(\varphi-\theta)+h \sin \varphi=0
$$

where $\mathrm{h}=\mathrm{H} / \mathrm{M}\left(\mathrm{D}_{\mathrm{b}}-\mathrm{D}_{\mathrm{a}}\right)=\mathrm{H} / \mathrm{H}_{\mathrm{A}}$, and here $\mathrm{H}_{\mathrm{A}}$ is the shape anisotropy. However, Eq. (4.19) is difficult to solve analytically. Thus, we just look at particular cases.

For $\mathrm{H}=0$, Eq. (4.19) indicates $\alpha=0$ and this means $\mathrm{M}$ lies along the polar axis. An applied field then rotates $M$ toward $H$. The component of $M$ along $H$ is $M \cos \varphi=M_{H}$. If $\mathrm{H}$ is applied perpendicular to the polar axis $(\theta=\pi / 2), h=\cos \varphi=H / H_{A}$ from Eq. (4.19). Therefore, magnetization $M_{H}$ changing linearly with field until $h=1$ (or $\left.H=H_{A}\right)$ means that there is no hysteresis because the rotation of the moment is reversible for this single domain particle. This relation is shown in Fig. 4.4 (a). Fig. 4.4 (b) shows H applied opposite to the polar axis $(\theta=\pi)$ leading the relation $\cos \varphi=-\mathrm{H} / \mathrm{H}_{\mathrm{A}}$. If field is applied opposite to the magnetization, some perturbation causes very small rotation of the magnetization, but the anisotropy will force $M$ to remain close to original orientation. Changes in magnetization can occur by jumps in a small field which will favor the magnetization to be in the field direction. Here, the coercivity $\mathrm{H}_{C}$ is $\mathrm{H}_{\mathrm{A}}$. 


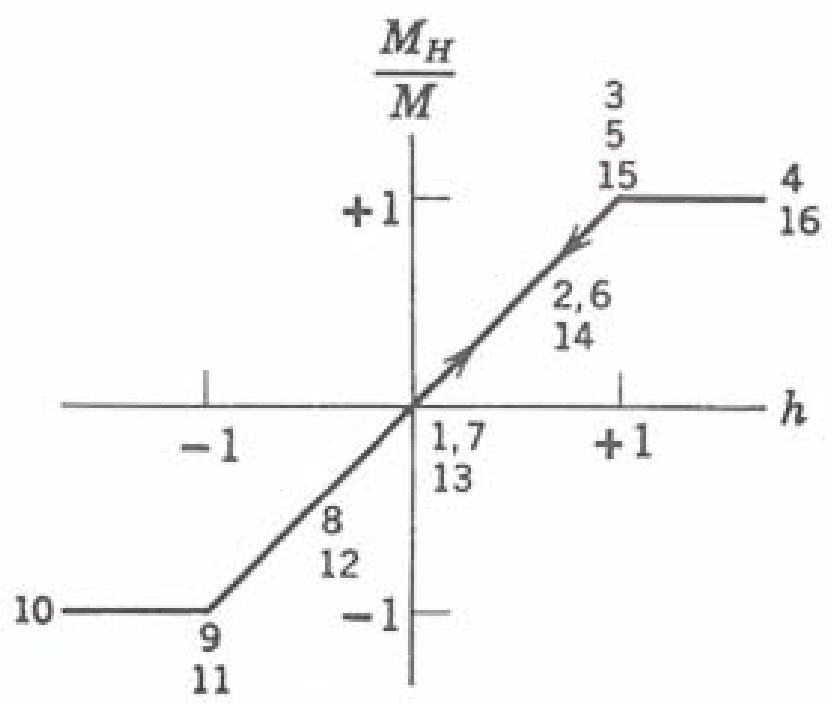

(a)

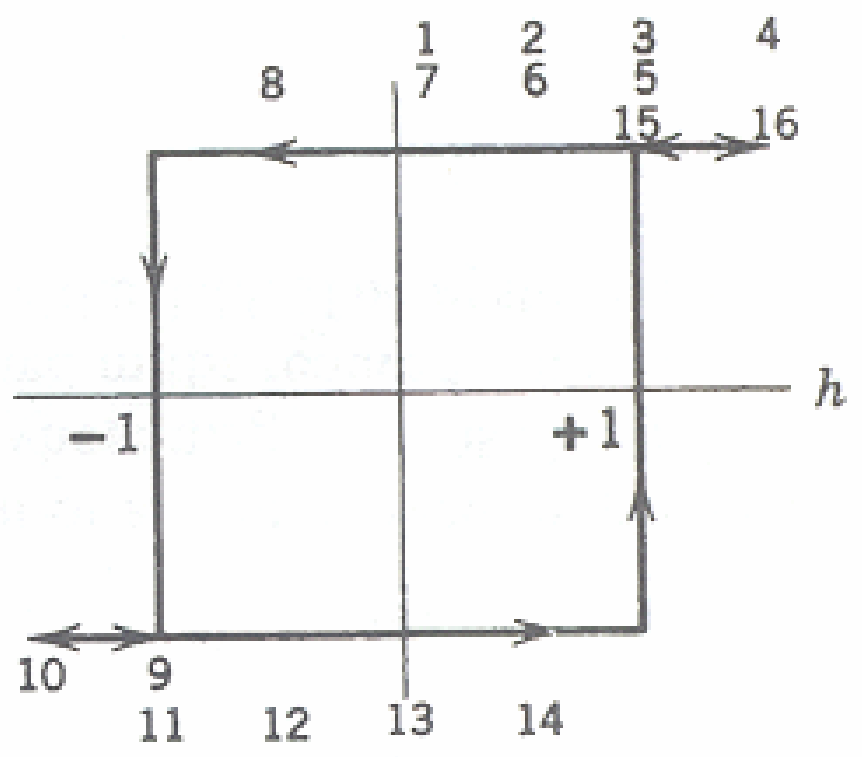

(b)

Fig. 4.4. (a) represents the case of a field applied perpendicular to the polar axis. There is a reversible rotation leading to no hysteresis, and (b) is the case of that a applied field is parallel with the polar axis. No reversible rotation occurs. 
In the case of $\theta=45^{\circ}$, the $\sin 2(\varphi-\theta)$ term of Eq. (4.19) equals $\cos 2 \varphi$ and hence double values of $\sin \varphi=\left\{-\mathrm{h} \pm\left(\mathrm{h}^{2}+2\right)^{1 / 2}\right\} / 2$ are calculated. Thus, there are jumps from $\varphi=30^{\circ}$ to $\varphi=-90^{\circ}$ at $\mathrm{h}=0.5$. This allows us to guess that the remanence and coercivity of an assembly of randomly oriented particles will be $\mathrm{M}_{\mathrm{H}} / \mathrm{M}=0.5$ and $\mathrm{h}_{\mathrm{C}}=$ $\mathrm{H} / \mathrm{H}_{\mathrm{C}}=0.5$ respectively since the coercivity field of the individual particles range from $\mathrm{h}$ $=0$ to $\mathrm{h}=1$. These values are comparable to the numerical calculation yielding $\mathrm{h}_{\mathrm{C}}=$ 0.479 and $\mathrm{M}_{\mathrm{H}} / \mathrm{M}=<\cos \varphi>=0.5$ [Stoner et al. 1948]. This is shown in Fig. 4.5.

\subsection{Relaxation and Magnetic Viscosity}

If a system is disturbed by an external perturbation, the time $\tau$ which it requires to return to the equilibrium state is the relaxation time. For magnetic nanoparticles, studies of relaxation time have been reported by a number of research groups. The relaxation time is determined by the interaction of the magnetic moment of the particles with its environment [Dormann et al 1980, 1983 and Fiorani et al 1981, 1983, 1986].

For isolated particles, the relaxation time is written as (Eq. (4.5)),

$$
\tau=\tau_{0} \exp \left(\Delta \mathrm{E} / \mathrm{k}_{\mathrm{B}} \mathrm{T}\right)
$$

where $\Delta \mathrm{E}=1 / 2 \mathrm{CV}=\mathrm{KV}$. This equation is called Néel-Arrhenius law which is valid only for non-interacting particles. However, in a real system, this relation has to be changed by including the effect of the influence of magnetic interactions on the relaxation time. In calculation of relaxation time for interacting particles, Shtrikman and Wohlfarth proposed one possible derivation which consisted of two cases, weak and strong coupling, respectively [Shtrikman et al, 1981]. For weak-coupling regime, they considered an energy barrier as $\mathrm{V}\left(\mathrm{K}+\mathrm{H}_{\mathrm{i}} \mathrm{M}\right)$ where $\mathrm{K}$ is the magnetocrystalline anisotropy constant and $\mathrm{VH}_{\mathrm{i}} \mathrm{M}$ is the contribution of the interaction energy to the barrier. Using a statistical mean value, $H_{i}$ is replaced by $\left(H_{i}{ }^{2} M V\right) / k_{B} T$. This leads above relaxation time to a VogelFulcher law,

$$
\tau \approx \tau_{0} \exp \left[\mathrm{VK} / \mathrm{k}_{\mathrm{B}}\left(\mathrm{T}-\mathrm{T}_{0}\right)\right]
$$

where $\mathrm{T}_{0}$ is proportional to $\mathrm{H}_{\mathrm{i}}{ }^{2}$ and increases with the interaction strength. If Eq. (4.21) is valid, then following the earlier arguments, it follows that

$$
\mathrm{T}_{\mathrm{B}}=\mathrm{T}_{0}+\left(\mathrm{KV} / \mathrm{k}_{\mathrm{B}}\right) / \ln \left(\mathrm{f}_{0} / \mathrm{f}_{\mathrm{m}}\right)
$$




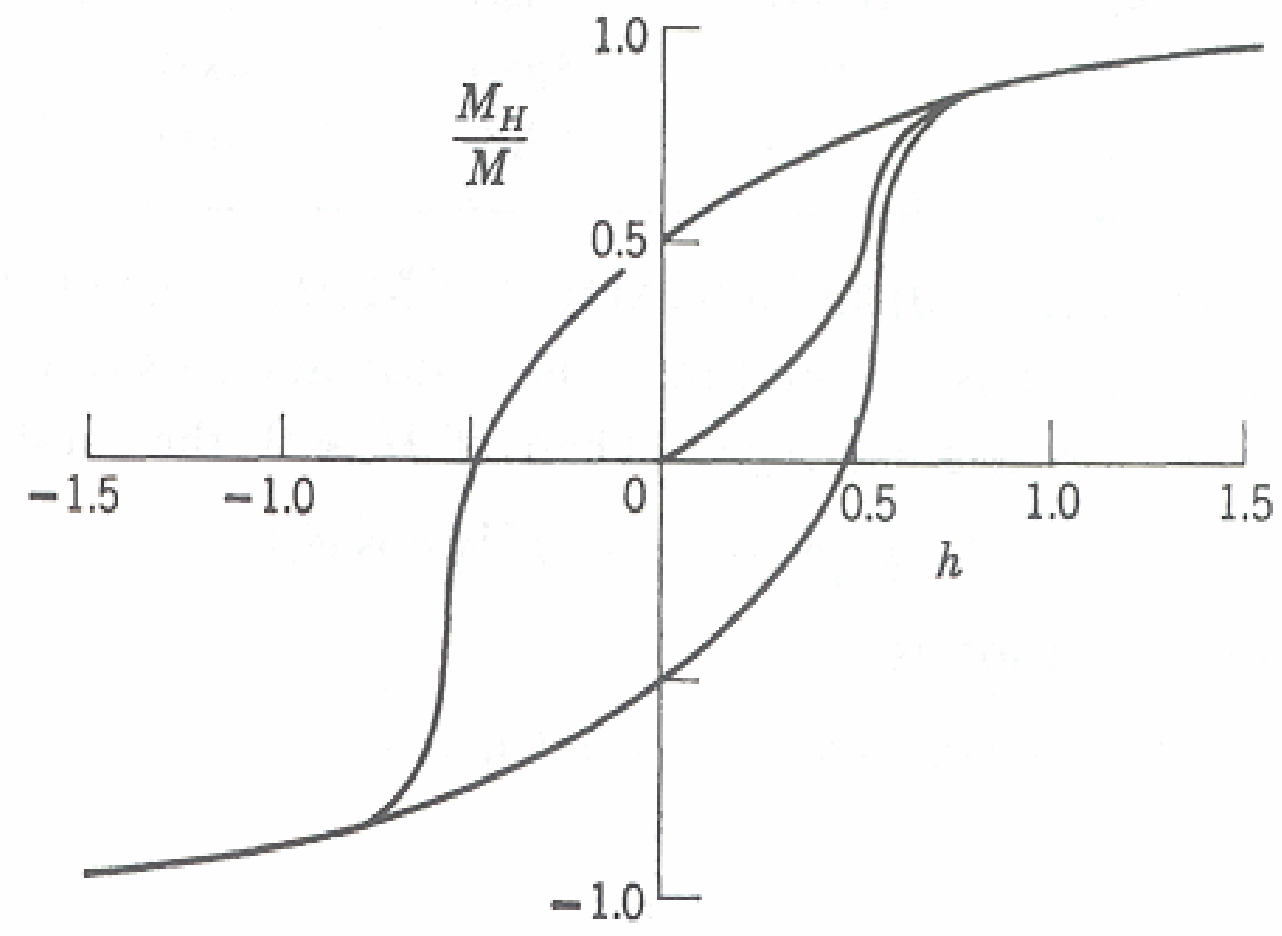

Fig 4.5 shows hysteresis curves of randomly oriented particles calculated by Stoner and Wohlfarth [Stoner et al. 1948]. 
Thus, the effect of the interaction represented by $T_{0}$ is to enhance $T_{B}$ by $T_{0}$ since the second term on the right hand side of Eq. (4.22) is $\mathrm{T}_{\mathrm{B}}$ of the non-interacting system at the measuring frequency $f_{m}$ (see Eq. (4.7)). A similar result was derived by Dormann et al. [Dormann et al. 1988].

The effect of an applied field $\mathrm{H}$ on $\mathrm{T}_{\mathrm{B}}$ of a non-interacting nanoparticle system for $\mathrm{H}$ applied along easy axis is to lower the energy barrier $\Delta \mathrm{E}=\mathrm{KV}$ to

$$
\Delta \mathrm{E} \rightarrow \Delta \mathrm{E}-\mu_{\mathrm{P}} \mathrm{H}+\mu_{\mathrm{P}}{ }^{2} \mathrm{H}^{2} / 4(\Delta \mathrm{E})
$$

where $\mu_{\mathrm{P}}$ is the magnetic moment of the particle [Bean and Livingston, 1959]. Ibrahim et al. [1995] have shown that the effect of Eq. (4.23) is to lower $T_{B}$ in the presence of an applied field $\mathrm{H}$. However, in real systems, the particles are oriented randomly with respect to $\mathrm{H}$ and the interaction of the particles has to be taken into account. I will discuss these issues in Chapter $\mathrm{V}$ when the experimental results are presented on $\mathrm{NiO}$ nanoparticles.

For the theoretical simple case, if we consider particles which have identical barriers, it will be easy to solve the problem of relaxation. When we apply a magnetic field to the particles, all the particles are aligned in one direction with the field direction. Then, as the field is removed, the magnetic moment of the particles will simply decay following Eq. (4.4). In reality, however, there is a broad distribution of energy barriers. In this case, the magnetization has been shown to decay as [Street et al, 1949],

$$
\mathrm{M}(\mathrm{t})=\mathrm{M}\left(\mathrm{t}_{0}\right)-\mathrm{S}(\mathrm{H}, \mathrm{T}) \ln \left(\mathrm{t} / \mathrm{t}_{0}\right)
$$

where $\mathrm{S}(\mathrm{H}, \mathrm{T})$ is the magnetic viscosity that depends on both the magnetic field $\mathrm{H}$ and the temperature T. A computer simulation study of the magnetic viscosity has been reported by Gonzalez et al. [Gonzalez et al. 1994] on a system with a distribution of barriers. This study showed that $\mathrm{S}(\mathrm{H}, \mathrm{T})$ peaks at a temperature $\approx \mathrm{T}_{\mathrm{B}}$. Experimental verification of this result has been observed in ferrihydrite nanoparticles [Ibrahim et al. 1995]. 


\section{CHAPTER V}

\section{Experimental Results in 5 nm NiO Nnanoparticles.}

In this chapter, I focus on our results for the smallest particles of $\mathrm{NiO}$ with $5 \mathrm{~nm}$ nominal XRD size and examine the effect of OA coatings on the magnetic properties.

\subsection{Electron Magnetic Resonance (EMR)}

In $5 \mathrm{~nm} \mathrm{NiO}$ nanoparticles, we observed an EMR signal at room temperature near $\mathrm{g} \approx 2$ at the EMR frequency $\mathrm{f}=9.28 \mathrm{GHz}$ following the resonance condition $\mathrm{hf}=\mathrm{g} \mu_{\mathrm{B}} \mathrm{H}_{\mathrm{r}}$. This is a surprising result since in bulk antiferromagnets, the EMR signal above $T_{N}$ disappears below $\mathrm{T}_{\mathrm{N}}$ because of the energy gap $\approx\left(2 \mathrm{H}_{\mathrm{E}} \mathrm{H}_{\mathrm{A}}\right)^{1 / 2}$ in the energy spectrum which appears for $\mathrm{T}<\mathrm{T}_{\mathrm{N}}$ [e.g. Seehra et al. 1970]. This EMR signal becomes weaker and unobservable for larger particle size. Although no definite explanation of the origin of the signal is known, there have been several suggestions such as the size effects [Kenning et al, 1987, Tang et al, 1991, and Sako et al, 1995], the uncompensated surface spins [Néel, 1962], the presence of a spin-glass-like shell on the particle surface [Martinez et al, 1998] and so on. Among these suggestions, the effect of uncompensated surface spins is regarded as the more likely possibility.

Recently, the temperature dependence of the EMR spectra of $6 \mathrm{~nm} \mathrm{NiO}$ particles was first reported by Rubinstein et al. [2001]. They showed that the resonance line was shifted to lower fields with decreasing temperature similar to ferrihydrite NP as reported by Seehra et al. [2001]. In the studies by Rubinstein et al [2001], the line width $\Delta H$ and the resonance field $\mathrm{H}_{\mathrm{r}}$ were difficult to measure because line shape was very complex and distorted. So interpretation of the data could not be given.

We performed the measurements of EMR signal with varied temperature and magnetic field for all the particles. EMR measurements reported here were done by Dr. P. Dutta of our research group [Seehra et al. 2004]. Our observations show that the strength

of the EMR signal decreases significantly as particle size increases. Only the uncoated and coated $5 \mathrm{~nm}$ samples have sufficiently well defined signals. Therefore, for $5 \mathrm{~nm}$ 
samples, the EMR studies were carried out by measuring various EMR parameters, such as line width $\Delta \mathrm{H}$, resonance field $\mathrm{H}_{\mathrm{r}}$ and intensity $\mathrm{I}_{0}$ as a function of temperature.

The EMR measurements were done at $9.28 \mathrm{GHz}$ using a standard reflection type cavity, a Varian magnet and an Oxford Instruments cryostat for variable temperature studies. The EMR measurements were done with decreasing temperature, starting from room temperature to $4 \mathrm{~K}$. For each temperature, the magnetic field was scanned from 100 to 10,100 Oe mimicking the field - cooled (FC) case.

The behavior of the EMR signal with temperature is shown in Fig. 5.1 for the uncoated $5 \mathrm{~nm}$ particles. From this graph, we can see that the lines broaden and shift to lower fields as temperature decreases. By analyzing each signal for both the uncoated and the coated samples, the comparative temperature dependences of the three EMR parameters, $\Delta H, H_{r}$ and $I_{0}$ are measured, as plotted in Fig. 5.2. First, it is noted that at each T, $\Delta H$ for the coated NP is reduced by almost a factor of two compared to that in the uncoated NP. This can be explained by the reduction of interparticle interaction due to the OA coating since any anisotropic interaction such as the dipolar interaction broadens the EMR signal [Castner et al, 1971 and references therein]. For the calculation of EMR intensity, ideally the intensity should be calculated by measuring the surface area under the curve by double integration of the derivative signals. In our case, however, as there is large zero-field absorption, this procedure will lead a wrong estimation while the equation $\mathrm{I}_{0}=(\Delta \mathrm{H})^{2} \ell, \ell$ being peak-to-peak height, provides a more reliable estimate of the intensity [Poole Jr. C.P, 1983].

For the experimental determination of $\mathrm{T}_{\mathrm{B}}(\mathrm{EMR})$ from the $\mathrm{I}_{0}$ vs. temperature plot, we can expect that for $T>T_{B}(E M R)$, the intensity $I_{0}$ will vary as $1 / T$ expected for superparamagnetism. For $\mathrm{T}<\mathrm{T}_{\mathrm{B}}(\mathrm{EMR})$, there will be a rapid decrease in magnitude of intensity with decreasing temperatures due to spin freezing. Following this criterion, we can easily estimate the blocking temperatures. Each sample shows two peaks regarded as blocking temperatures, $T_{B}(m)$ measured by magnetization studies and $T_{B}(E M R)$ measured by EMR studies. $T_{B}(m)$ is at lower temperature than $T_{B}(E M R)$ as EMR signal is measured in high frequency. These two blocking temperatures for the uncoated sample vs. the coated sample are shifted to a higher temperature region by a temperature difference $\approx 140 \mathrm{~K}$ presumably due to strong interparticle interaction. The field 
dependence of the blocking temperature and the ratio of these two blocking temperatures are more specifically discussed later.

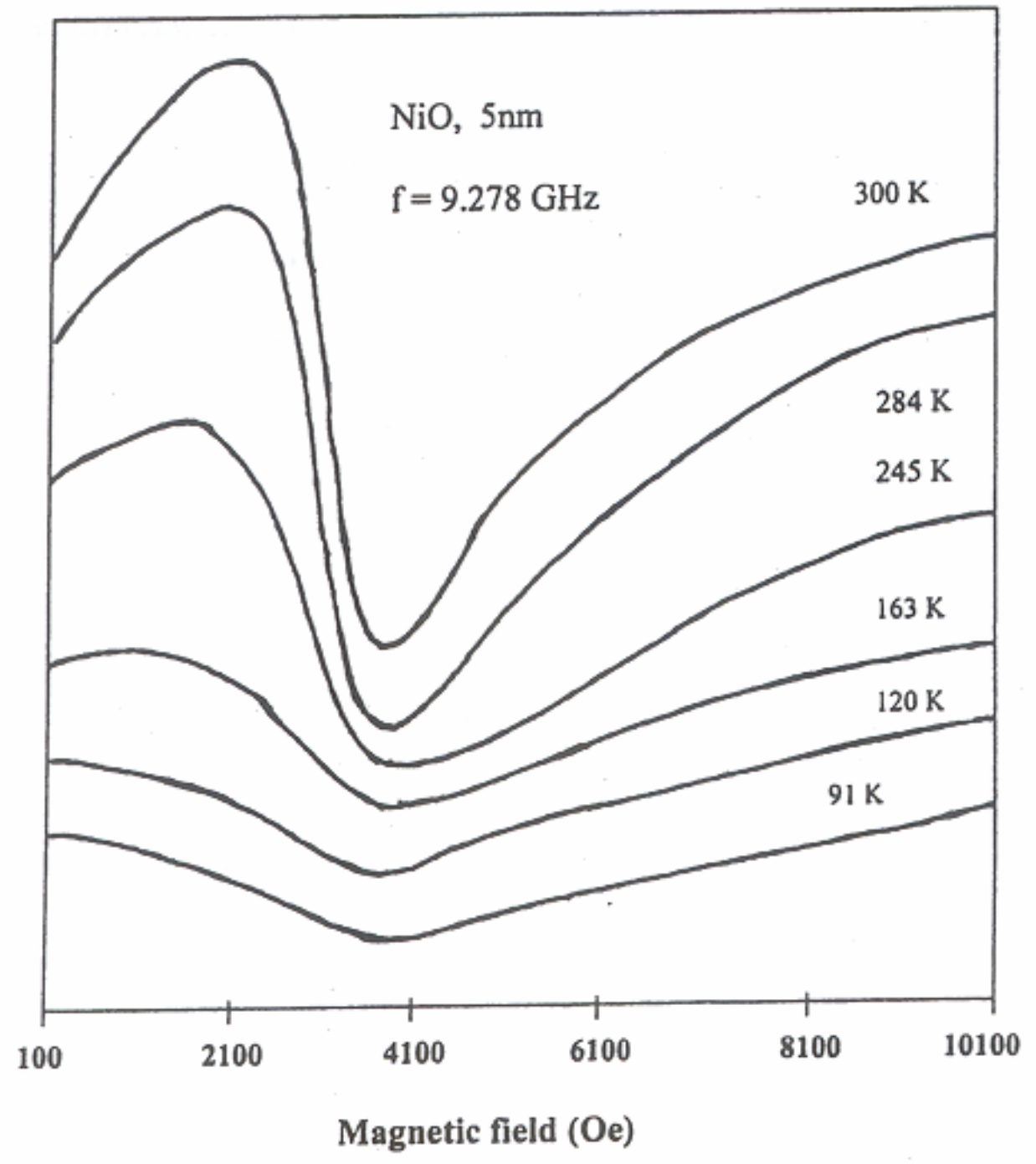

Fig. 5.1 Plots of the EMR derivative line for several temperatures for the uncoated NiO. 


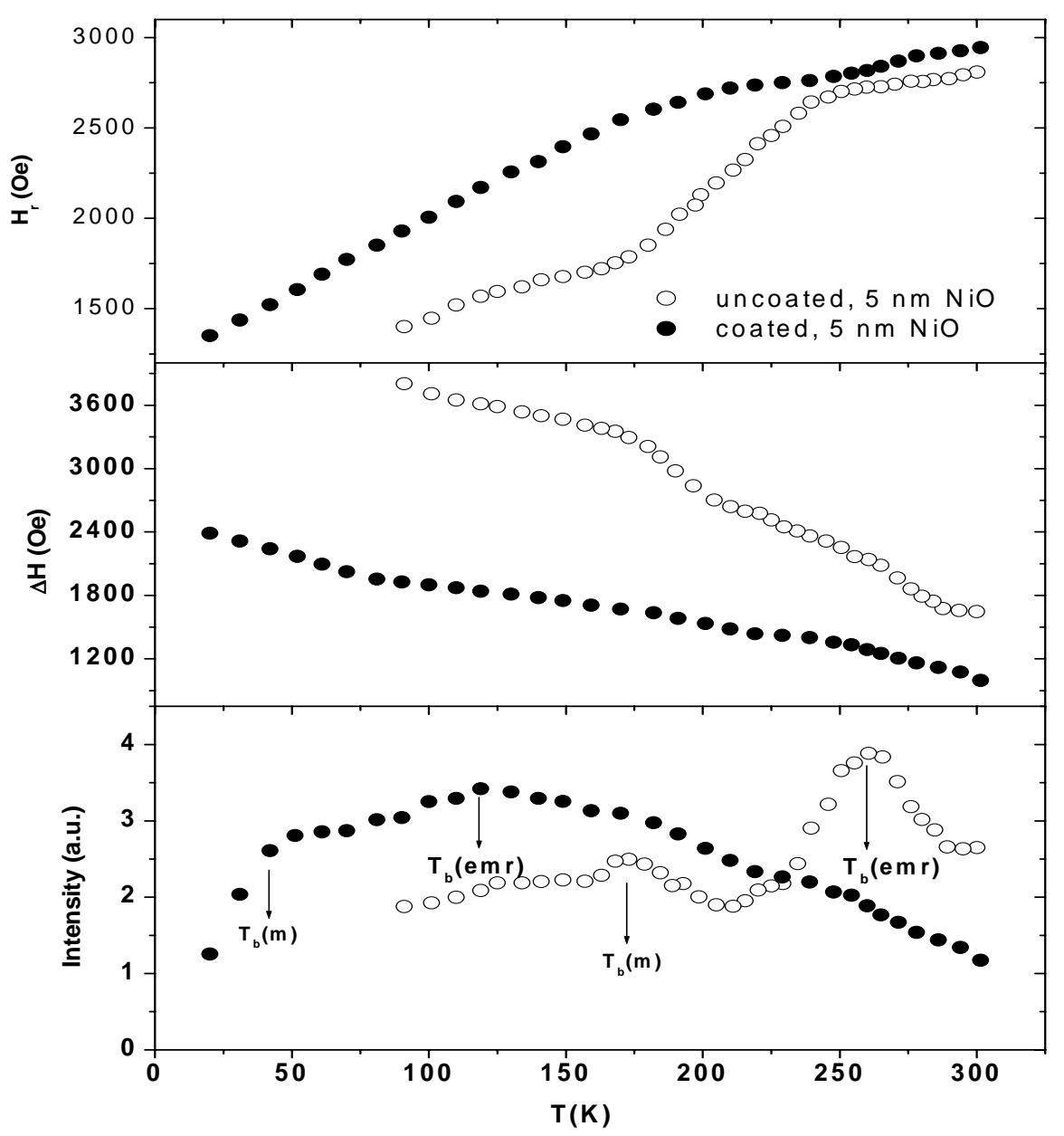

Fig. 5.2 Plots of the resonance field $\mathrm{H}_{\mathrm{r}}$, linewidth $\Delta \mathrm{H}$, and intensity $\mathrm{I}_{0}$ of the EMR line against temperature for the coated and uncoated $\mathrm{NiO}$. 


\subsection{Temperature Variation of the low-field magnetic susceptibility}

The susceptibility $\chi$ versus $\mathrm{T}$ was measured in zero-field-cooled (ZFC) condition and field-cooled (FC) for both the coated and uncoated nanorods. Each sample is cooled from room temperature to $5 \mathrm{~K}$ with $\mathrm{H}=0$ and then the magnetization $\mathrm{M}$ was measured at $\mathrm{H}=100 \mathrm{Oe}$. This is the ZFC measurement. The FC measurements used a similar procedure except the samples were cooled to $5 \mathrm{~K}$ in $\mathrm{H}=100$ Oe. Prior to the SQUID measurements, we made a small sample holder using a white plastic drinking straw. The magnetization data were corrected to remove the background effect of the sample holder with $\chi=\mathrm{M} / \mathrm{H}=-2.3 \times 10^{-8}$, independent of $\mathrm{T}$.

The plots of the variation of $\chi$ vs. $T$ for the uncoated (a) and the coated cases (b) are shown in Fig. 5.3 with $\mathrm{T}_{\mathrm{P}}$ as the temperature at which $\chi(\mathrm{ZFC})$ peaks. In the temperature regions above $\mathrm{T}_{\mathrm{P}}$, the $\mathrm{ZFC}$ and $\mathrm{FC}$ curves are nearly identical and approximately follow the Curie-law. However, the curves are not identical due to the particle size distribution observed in our TEM analysis. Comparing the data for the two samples, we note two differences. The first is that the average blocking temperature $T_{B}$, proportional to $T_{P}$ in $\chi$ (ZFC) shifted to lower $T$ for coated NP. The temperature difference is around $140 \mathrm{~K}$ and similar to the difference in EMR data between the uncoated and the coated samples. The second point is that for the uncoated NP, the FC curve is flattened out at lower $\mathrm{T}$. These two results are interpreted in terms of reduction of the interparticle dipolar interaction upon OA coating [Bødker et al. 2000, Chantrell et al. 2000, and Mørup 1994], as discussed in further detail later.

In Chapter IV, we derived the equation for $T_{B}$ as

$$
\mathrm{T}_{\mathrm{B}}=\left(\Delta \mathrm{E} / \mathrm{k}_{\mathrm{B}}\right) / \ln \left(\mathrm{f}_{0} / \mathrm{f}_{\mathrm{m}}\right)
$$

For our SQUID measurements, the measuring frequency $\mathrm{f}_{\mathrm{m}} \approx 1 \mathrm{~Hz}$ and for previous EMR, $\mathrm{f}_{\mathrm{m}} \approx 9.28 \times 10^{9} \mathrm{~Hz}$ was used. Using these two frequencies and above equation, we can calculate the theoretical ratio of $\mathrm{T}_{\mathrm{B}}(\mathrm{EMR}) / \mathrm{T}_{\mathrm{B}}(\mathrm{m})$ as $5.9(4.3)$ for $\mathrm{f}_{0}=10^{12}\left(10^{13}\right) \mathrm{Hz}$ for non-interacting SPM particles. It is known theoretically that the ratio $T_{B}(E M R) / T_{B}(m)$ decreases as the strength of the interparticle interaction increases [Mørup, 1994]. In our experiments, the obtained the ratio, $\mathrm{T}_{\mathrm{B}}(\mathrm{EMR}) / \mathrm{T}_{\mathrm{B}}(\mathrm{m}) \approx 3.1(1.5)$ for the coated (uncoated) 
NP. These values are in line with the theoretical estimates discussed above [Mørup, 1994].

\subsection{Field Dependence of Blocking Temperatures}

To study the effects of interparticle interactions further, we measured the blocking temperatures in ZFC for different measuring fields, from 25 Oe through 1,000 Oe. The shift in $\mathrm{T}_{\mathrm{B}}(\mathrm{m})$ with applied field $\mathrm{H}$ is shown in Fig. 5.4 for uncoated and coated NP. Normalized plots of $\mathrm{T}_{\mathrm{B}}(\mathrm{H}) / \mathrm{T}_{\mathrm{B}}(25 \mathrm{Oe})$ versus $\mathrm{H}$ are shown in Fig. 5.5 indicating a decrease of the $\mathrm{T}_{\mathrm{B}}(\mathrm{H})$ with increasing field. As discussed in Chapter IV, the applied field reduced the energy barrier for relaxation and hence $T_{B}$ is reduced. It is evident that decrease for coated NP is more pronounced for smaller $\mathrm{H}$ than that for uncoated NP. Therefore we can expect that for non-interacting NP, the change in the energy barrier with field is much larger than that for interacting NP. This phenomenon is theoretically discussed by El-Hilo et al. [1992] through a computer simulation taking into account the effect of interparticle interaction. In Fig. 5.4, we also notice that a second peak is observed for the coated NP in the lower temperature region around $\mathrm{T} \approx 13 \mathrm{~K}$. This peak may be from the OA coating since it does not change with applied $\mathrm{H}$. Therefore no further discussion of this peak is presented here.

Several measurements have been reported on the field dependence of $T_{B}$ for a fine particle system. Using Brown's equation, Wenger and Mydosh showed that for a constant value of $\tau$, the dependence of $\mathrm{T}_{\mathrm{B}}$ on $\mathrm{H}$ is of the form, $\mathrm{T}_{\mathrm{BH}} \propto \mathrm{H}^{v}$, where $v=2$ for low fields and $v=2 / 3$ for high fields [Wenger et al, 1984]. For low fields, they derived the following equation,

$$
\mathrm{T}_{\mathrm{BH}}=\mathrm{T}_{\mathrm{B} 0}\left[1-\mathrm{I}_{\mathrm{sb}} \mathrm{VH}^{2} / \mathrm{k}_{\mathrm{B}} \mathrm{H}_{\mathrm{K}} \mathrm{T}_{\mathrm{B} 0}\right]
$$

where $T_{B H}$ and $T_{B 0}$ are the characteristic blocking temperatures in an applied field and zero field, indicating the $\mathrm{H}^{2}$ dependence. For high fields, they derived $\mathrm{H}^{2 / 3}$ variation, although an analytical expression for all $\mathrm{H}$ was not possible. Similar results were derived by Dormann. 


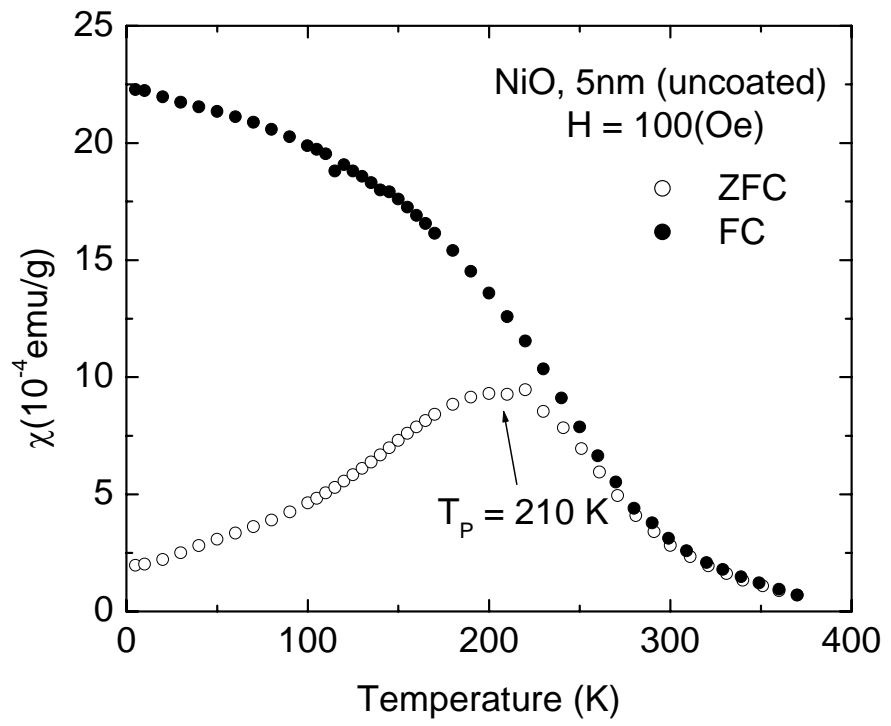

(a) uncoated

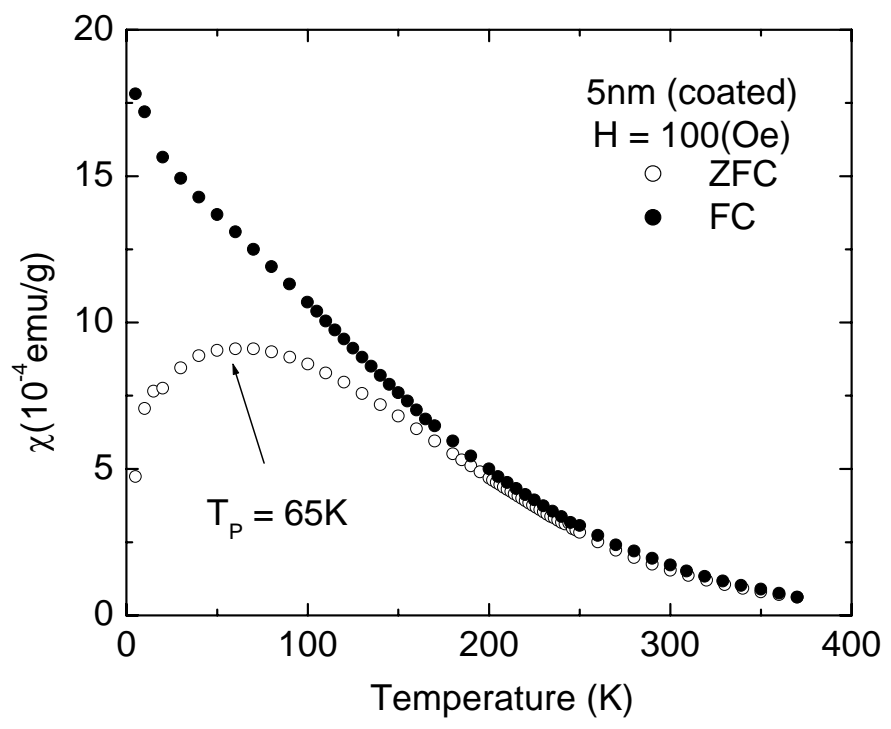

(b) coated

Fig. 5.3 Temperature dependence of the magnetic susceptibility for FC (field-cooled) and ZFC (zero-field cooled) cases showing reduction in blocking temperature by coating. 

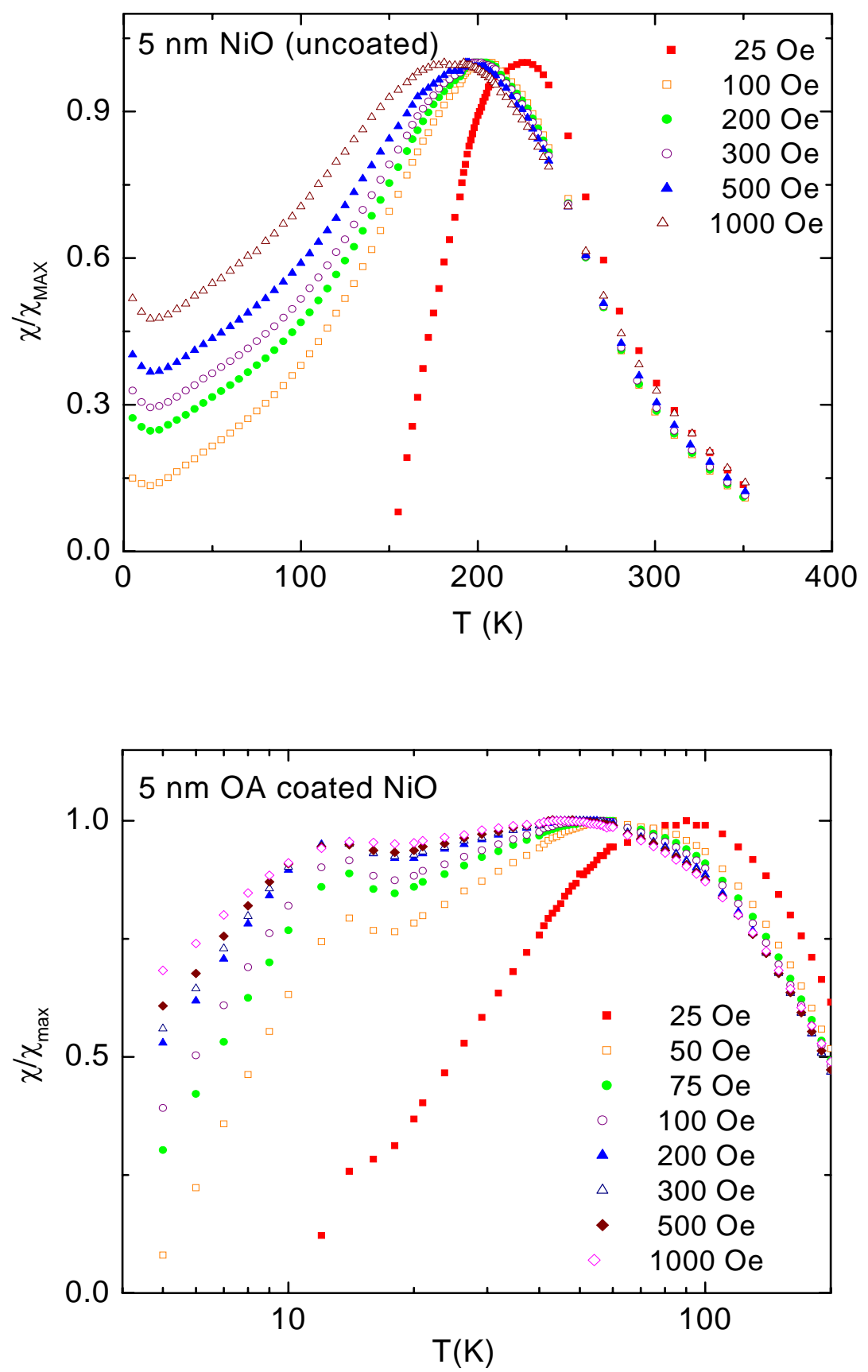

Fig. 5.4 Plot of $\chi / \chi_{\max }\left(\chi_{\max }\right.$ is the value at $\mathrm{T}=\mathrm{T}_{\mathrm{B}}$ defined by the maximum) in various applied fields against temperature for coated and uncoated NP. 


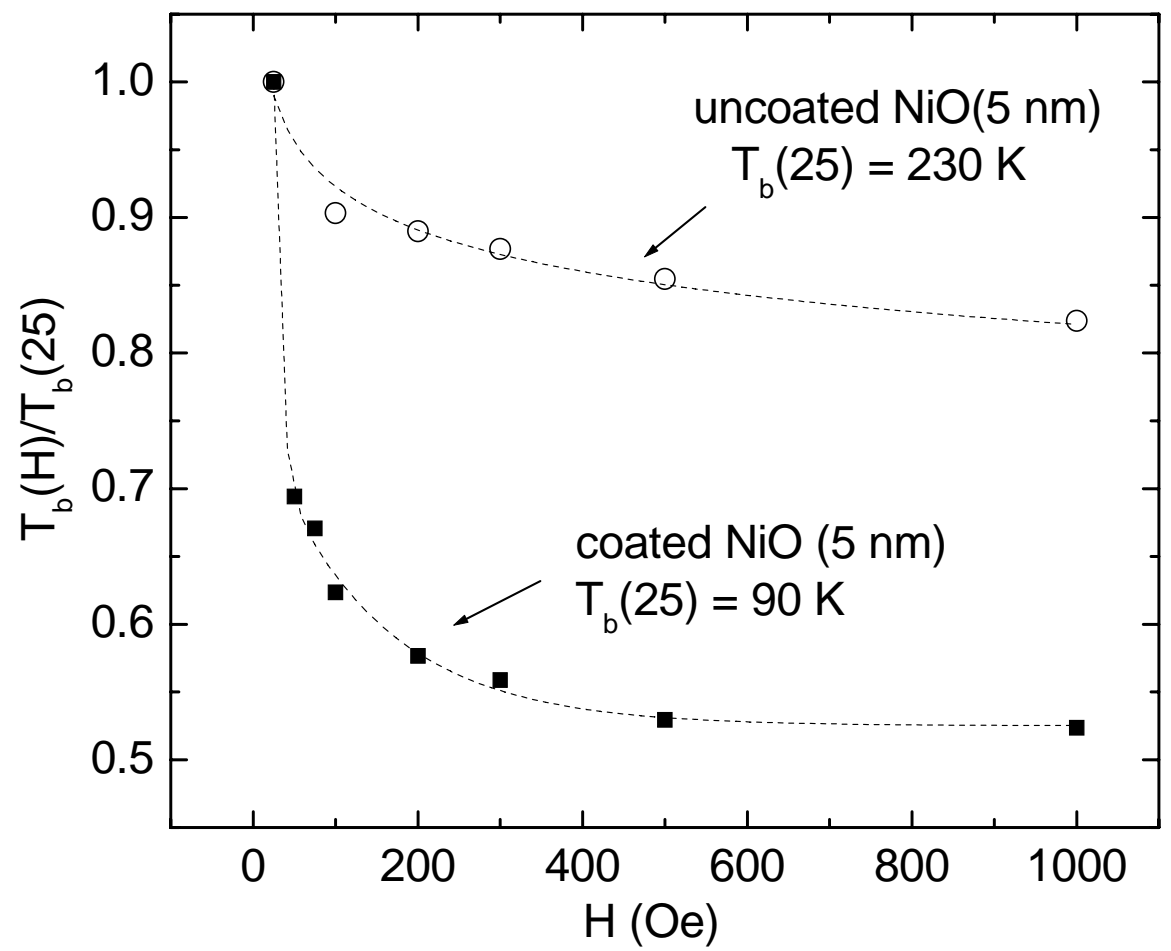

Fig. 5.5 Plots of the blocking temperature $\mathrm{T}_{\mathrm{B}}(\mathrm{H})$ at a magnetic field $\mathrm{H}$ normalized by its value at $\mathrm{H}=25 \mathrm{Oe}$ vs. $\mathrm{H}$. The dotted lines are visual guides. 
In a more recent paper, El-Hilo et al. [1992] have calculated the effect of interparticle dipolar interaction on the variation of $T_{B}$ with applied field $H$. They compared the results of computer simulation data with experimental data for a weakly interacting fine particle system, $\mathrm{Fe}_{3} \mathrm{O}_{4}$. Fig. 5.6 shows that the variation of the normalized blocking temperature, $\mathrm{T}_{\mathrm{RH}}$ is proportional to $\mathrm{H}^{2 / 3}$. Their data have a good agreement with the theoretical prediction showing $\mathrm{T}_{\mathrm{RH}} \propto \mathrm{H}^{2 / 3}$ at high fields and $\mathrm{T}_{\mathrm{RH}} \propto \mathrm{H}^{2}$ at low fields and the effect of interaction is to weaken the field dependence.

Tiwari and Rajeev [Tiwari et al. 2005] have used the observed $\mathrm{H}^{2 / 3}$ variation of $\mathrm{T}_{\mathrm{P}}$ as evidence that $\mathrm{T}_{\mathrm{P}}$ in $\mathrm{NiO}$ represents spin-glass freezing temperature. However, as discussed earlier, it has been shown theoretically that the $\mathrm{H}^{2 / 3}$ variation is valid not only for the mean-field models of spin-glasses but also for superparamagnetic particles, although for the latter cases, the variation is expected to change to $\mathrm{H}^{2}$ in the limit $\mathrm{H} \rightarrow 0$ [Wenger et al. 1989 and Dormann et al. 1987]. Furthermore, it has been shown that the effect of dipolar interparticle interaction is to weaken the $\mathrm{H}$ dependence [El-Hilo et al. 1992] and this has been verified in nanoparticles of $\mathrm{Fe}_{3} \mathrm{O}_{4}$ [El-Hilo et al. 1992] and in our studies of the $5 \mathrm{~nm} \mathrm{NiO}$ nanorods [Seehra et al. 2005]. To check the $\mathrm{H}^{\mathrm{q}}$ dependence in our $5 \mathrm{~nm}$ NiO-NP, we have fit the data to the Eq: $\mathrm{T}_{\mathrm{P}}(\mathrm{H})=\mathrm{T}_{\mathrm{P}}(0)\left[1-\mathrm{CH}^{\mathrm{q}}\right]$ where $\mathrm{C}$ is a constant. The results are shown in Fig. 5.7 as plots of $\ell n\left[\mathrm{~T}_{\mathrm{P}}(0)-\mathrm{T}_{\mathrm{P}}(\mathrm{H})\right]$ vs. $\ell n \mathrm{H}$ as well as $\left[T_{P}(0)-T_{P}(H)\right]$ vs. $H^{2 / 3}$. For lower fields, $q$ is larger but the magnitudes of $q$ are clearly smaller than the theoretically expected values of $q=2(2 / 3)$ for lower (higher) $\mathrm{H}$. Interestingly, in the plot of $\mathrm{H}^{2 / 3}$ variation in Fig. 5.7, one might infer that the variation is linear for larger $\mathrm{H}^{2 / 3}$. However, this is misleading since $\ell n\left[\mathrm{~T}_{\mathrm{P}}(0)-\mathrm{T}_{\mathrm{P}}(\mathrm{H})\right]$ vs. $\ell n \mathrm{H}$ plot yields entirely different magnitudes of $q$. It is evident that $H$ dependence of $T_{P}$ in our samples is quite different form that reported by Tiwari and Rajeev [Tiwari et al. 2005]. 


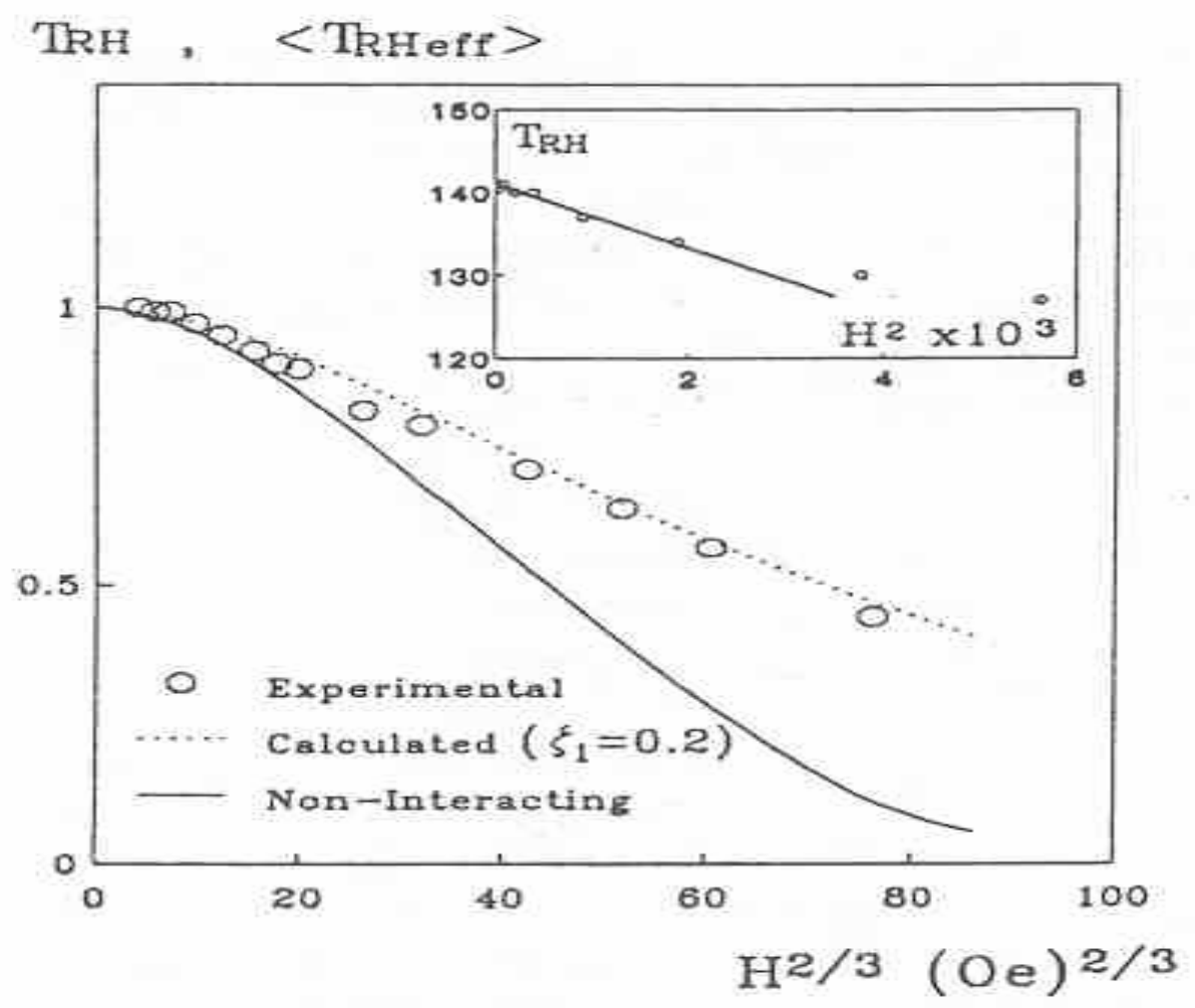

Fig. 5.6 The measured variation of the normalized temperature, $\mathrm{T}_{\mathrm{RH}} \mathrm{Vs} . \mathrm{H}^{2 / 3}$. The dotted line and solid lines are interacting and non-interacting cases respectively [El-Hilo, 1992]. 

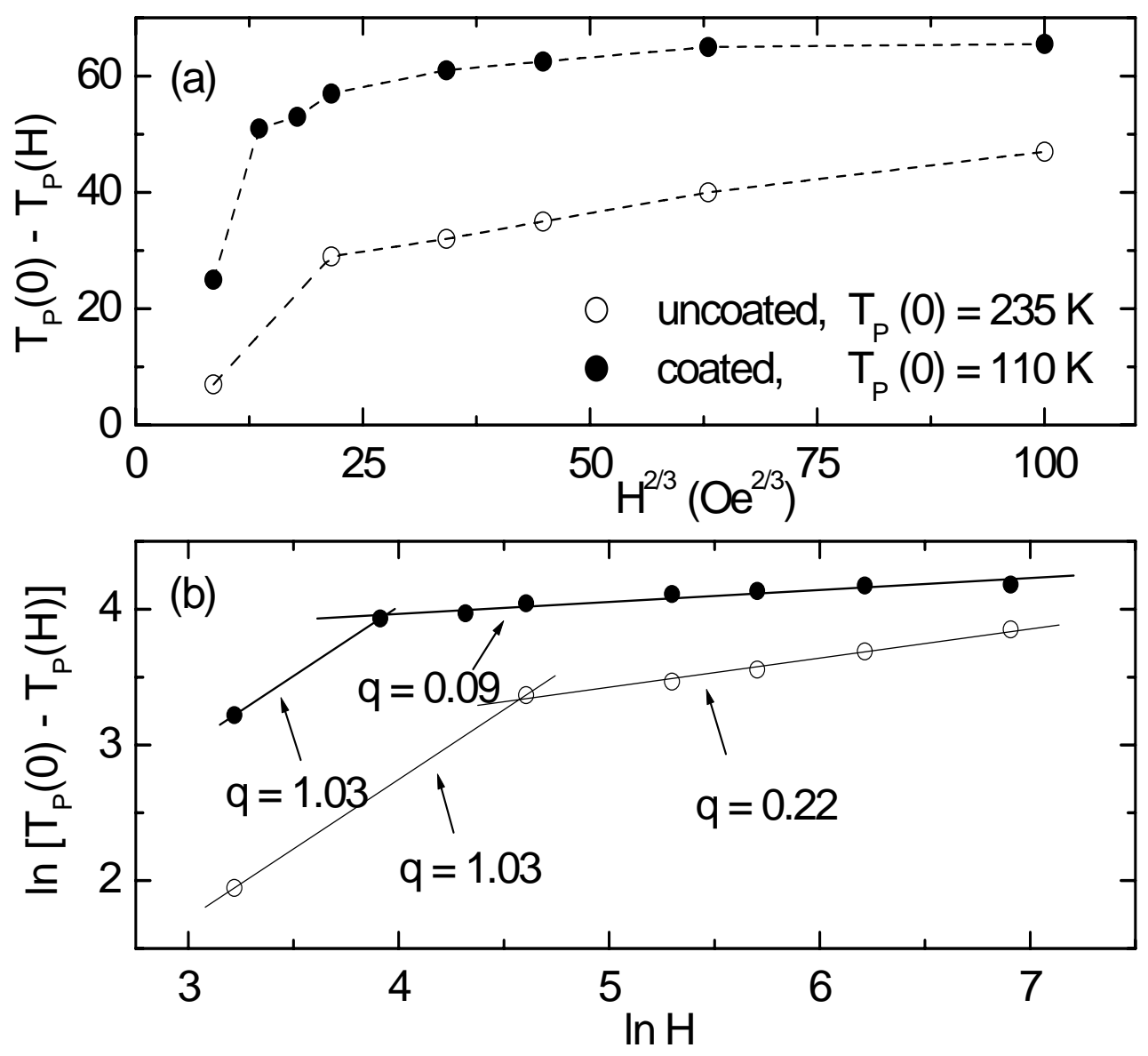

Fig. 5.7 (a) Plot of the shift in the peak temperatures $\left[T_{P}(0)-T_{P}(H)\right]$ against $H^{2 / 3}$ for the $5 \mathrm{~nm} \mathrm{NiO}$ particles. The lines connecting the points are for visual aid. (b) Plot of ln $\left[\mathrm{T}_{\mathrm{P}}(0)-\mathrm{T}_{\mathrm{P}}(\mathrm{H})\right]$ against $\ell n \mathrm{H}$ to check the $\mathrm{H}^{\mathrm{q}}$ variations. 


\subsection{Magnetization $M$ vs. Field $H$}

In the AF superparamagnetic system, the magnetization $M$ above the blocking temperature is expected to follow the modified Langevin function shown as in Eq. (4.16). From the dc susceptibility measurement in the previous section, we know $T_{B}$ for the coated and uncoated NP are $65 \mathrm{~K}$ and $210 \mathrm{~K}$ respectively, at the measuring field $\mathrm{H}=100$ Oe. The measurements of the magnetization $\mathrm{M}$ are performed with field $\mathrm{H}=0$ through $55 \mathrm{kOe}$ at various temperatures of 230 to 370 (300) K for uncoated (coated) samples. Fig. 5.8 shows the experimental data. Following Eq. (4.16), the data fit well to the Langevin function giving one single curve for all the measured temperatures as shown in Fig. 5.9. From this fitting, we obtained magnetic moments per particle, $\mu_{\mathrm{P}} \approx 1000(1240) \mu_{\mathrm{B}}$ for coated (uncoated) NP of nominal XRD size $\approx 5 \mathrm{~nm}$.

Below the blocking temperature $\mathrm{T}_{\mathrm{B}}$, the system is expected to be in the blocked (ordered) state having a hysteresis feature. The hysteresis loops for both coated and uncoated NP were measured at $5 \mathrm{~K}$ using $\mathrm{ZFC}$ and $\mathrm{FC}$ methods up to $\mathrm{H}= \pm 55 \mathrm{kOe}$ respectively. For the $\mathrm{FC}$ case, the samples were cooled to $5 \mathrm{~K}$ in $\mathrm{H}=20 \mathrm{kOe}$, followed by the hysteresis loop measurements. Fig. 5.10 shows the hysteresis curves of the FC in an expanded scale to compare the coercivity $\mathrm{H}_{\mathrm{C}}$ and exchange bias $\mathrm{H}_{\mathrm{E}}$ for both the uncoated and the coated particles. For the uncoated NP, there is reduction of $\mathrm{H}_{\mathrm{C}} \mathrm{vs}$. the coated NP. It may be explained in terms of the effect of interparticle interaction. Kechrakos et al. [1998] using Monte Carlo computer simulation have shown that for a weakly dipolar interacting system at low temperature, there is a reduction of the coercivity with increasing interparticle interaction. Qualitatively, the interparticle interaction averages out the anisotropy $\mathrm{H}_{\mathrm{A}}$ and thus reduces the coercivity $\mathrm{H}_{\mathrm{C}}$ which is proportional to $\mathrm{H}_{\mathrm{A}}$. 

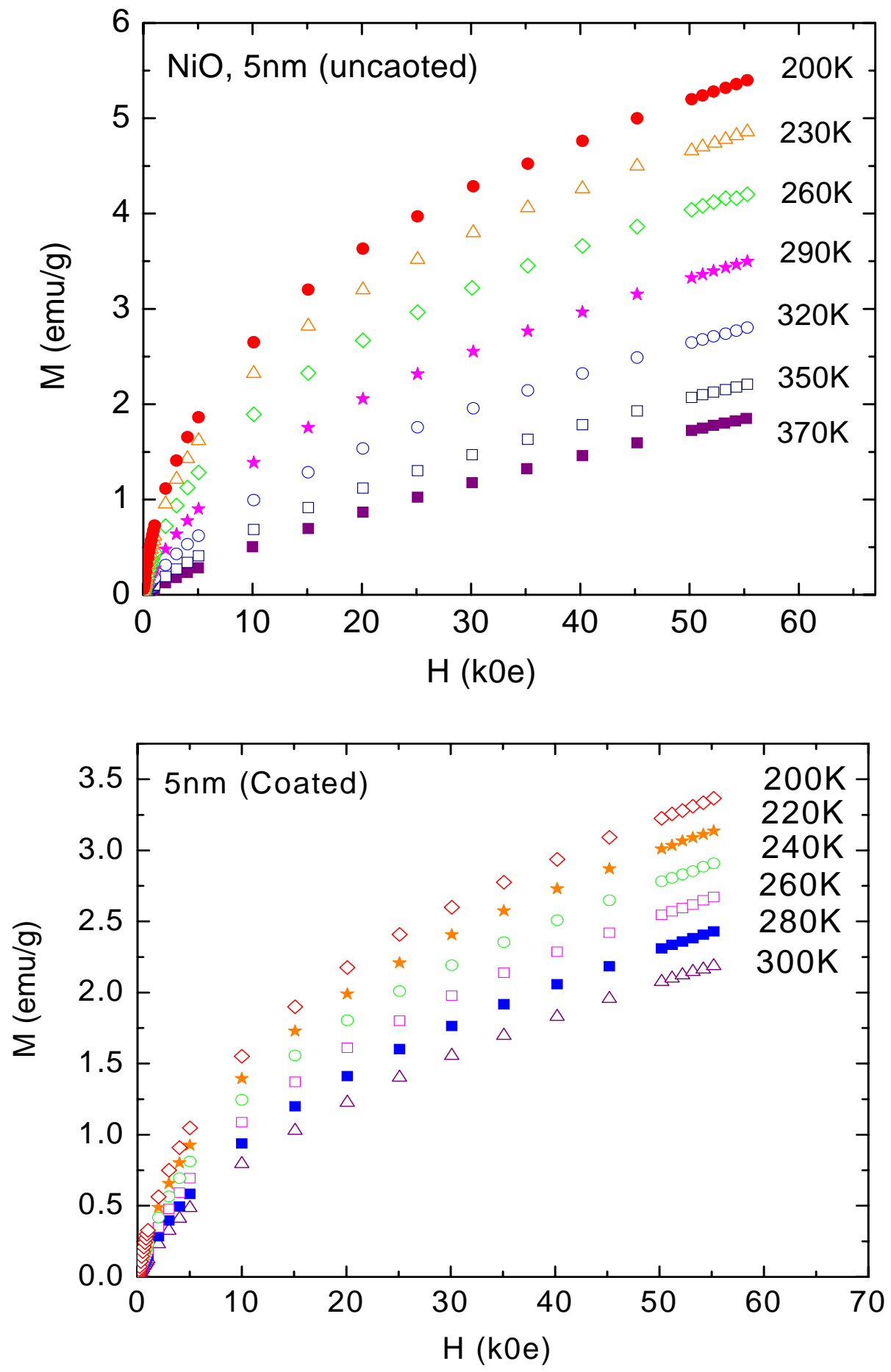

Fig. 5.8 Isothermal plots of the magnetization $\mathrm{M}$ against applied field $\mathrm{H}$ at temperatures shown. 

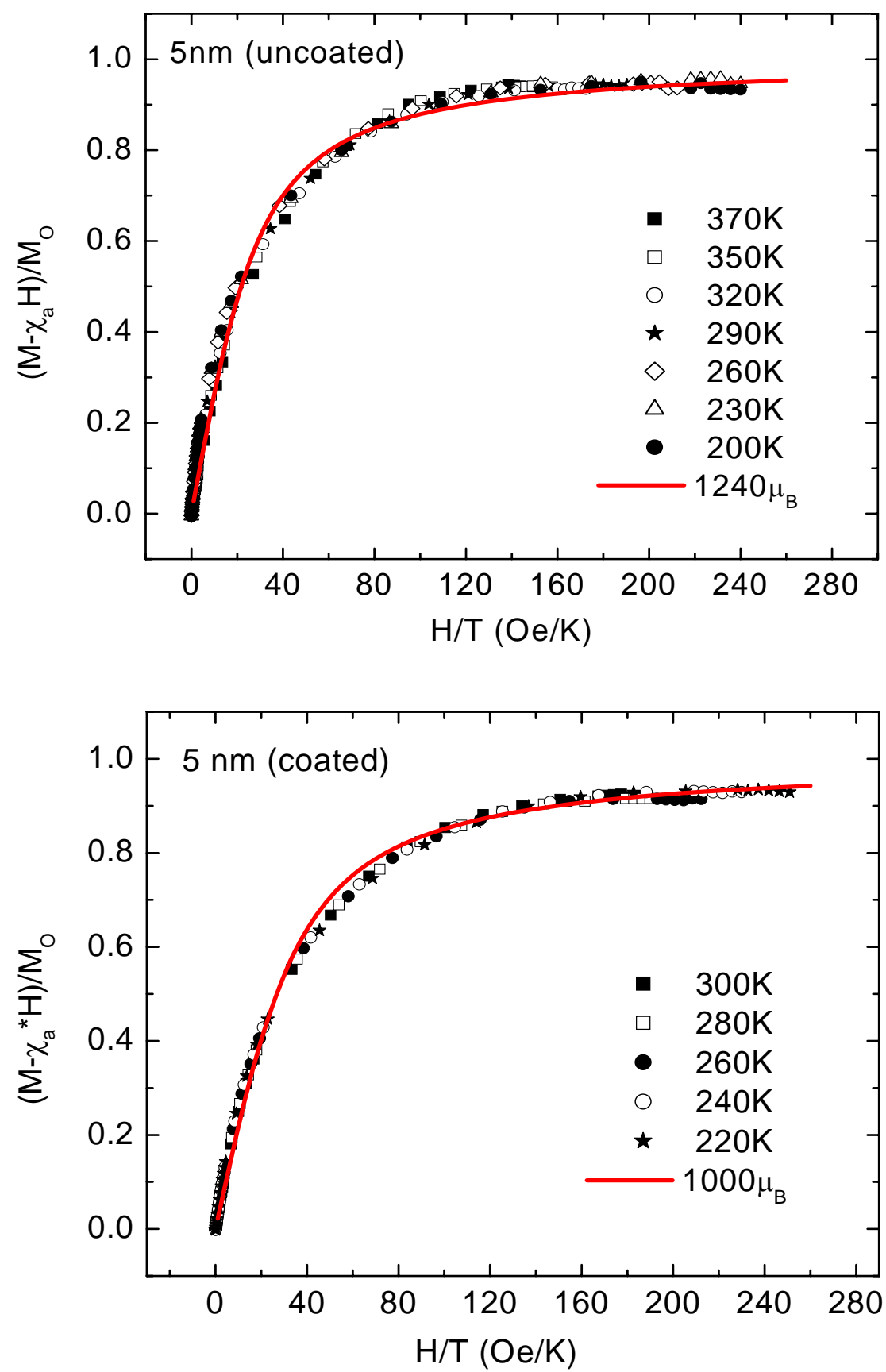

Fig. 5.9 Fitting of the $M$ vs. $H$ data to Eq. (4.16) yielding the plot of $\left(M-\chi_{a} H\right) / M_{0}$ vs. $\mathrm{H} / \mathrm{T}$. The solid line is the Langevin variation with $\mu_{\mathrm{P}}=1240(1000) \mu_{\mathrm{B}}$ for uncoated (coated) NP. 


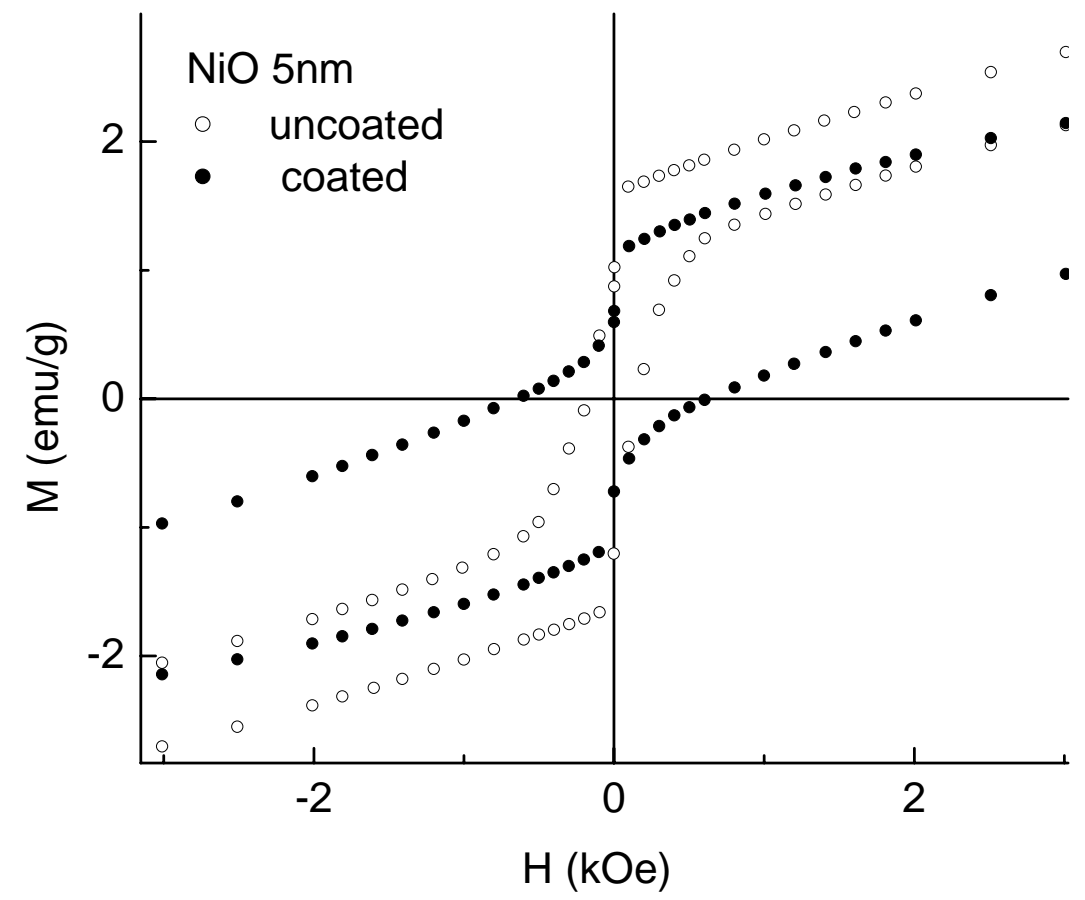

Fig. 5.10 Plot of $\mathrm{M}$ vs. $\mathrm{H}$ measured at $\mathrm{T}=5 \mathrm{~K}$ shows that the coercivity $\mathrm{H}_{\mathrm{C}}$ is smaller for the uncoated particles vs. the coated particles. 


\subsection{Frequency Dependence of Blocking Temperatures}

Here, I present the results of the AC measurements of the magnetic susceptibilities, $\chi^{\prime}$ and $\chi^{\prime \prime}$, using the frequencies $\mathrm{f}_{\mathrm{m}}=0.1 \mathrm{~Hz}$ to $10^{4} \mathrm{~Hz}$. Interpretation of these results discussed later shows that the relaxation rates are governed by the VogelFulcher law,

$$
\mathrm{f}=\mathrm{f}_{0} \exp \left\{-\mathrm{E}_{\mathrm{a}} / \mathrm{k}_{\mathrm{B}}\left(\mathrm{T}_{\mathrm{B}}-\mathrm{T}_{0}\right)\right\}
$$

where $\mathrm{T}_{0}=162(0) \mathrm{K}$ for the uncoated (coated) particles. Measurements of the AC magnetic susceptibilities $\chi^{\prime}$ and $\chi^{\prime \prime}$ were carried out using commercial SQUID magnetometers in 7 Oe amplitude of the AC field but zero DC magnetic field. The data above $1 \mathrm{kHz}$ were taken using the physical property measurement system (PPMS) at Bose State University whereas the data at the lower frequencies were taken using the magnetic property measurement system (MPMS) at West Virginia University [Shim et al. 2006].

Fig. 5.11 shows the plots of $\chi^{\prime}$ and $\chi^{\prime \prime}$ against temperature for the uncoated $5 \mathrm{~nm}$ NP. The plot of $\chi^{\prime}$ versus temperature for the coated NP is shown in Fig. 5.12. The $\chi^{\prime \prime}$ vs. T plot had large scatter because of small magnitudes and so this data are not shown here. We can see that the temperature position of the peak of $\chi^{\prime}$ is much higher than that of $\chi^{\prime \prime}$ and from a closer examination, the position of the peak of $\chi^{\prime \prime}$ agrees with the peak position of $\mathrm{d}\left(\chi^{\prime}\right) / \mathrm{dT}$. Similar observations have been reported in other NP systems [Dormann et al, 1996, Jonsson et al, 1998, and Vincent et al, 1996]. For both NP, the peak positions of $\chi^{\prime}$ and $\chi^{\prime \prime}$ shift to higher temperatures with increasing frequency as we expect from the above VF law. Note that the second peak is observed again for the coated $\mathrm{NP}$ in the lower temperature region, near $\mathrm{T} \approx 13 \mathrm{~K}$, similar to the case of the measurements of the field dependence of blocking temperature. These second peaks in the coated NP might be due to the OA coating.

From Eq. (5.3), it follows that

$$
\ln \mathrm{f}=\ln \mathrm{f}_{0}-\left(\mathrm{E}_{\mathrm{a}} / \mathrm{k}\right) /\left(\mathrm{T}_{\mathrm{B}}-\mathrm{T}_{0}\right) .
$$

For $\mathrm{T}_{0}=0$, Eq. (5.3) reduces to the Néel-Arrhenius (NA) relation, with

$$
\ln \mathrm{f}=\ln \mathrm{f}_{0}-\left(\mathrm{E}_{\mathrm{a}} / \mathrm{k}\right) / \mathrm{T}_{\mathrm{B}}
$$


First, in order to check the validity of NA relation for our coated and uncoated NP, we plot $\ln f$ vs. $1 / T_{B}$ in Fig. 5.13 using the peak position in $\chi^{\prime}$ to be $T_{B}$ and the value of $T_{B}$ obtained from the EMR studies at $\mathrm{f}=9 \times 10^{9} \mathrm{GHz}$ [Seehra et al, 2005]. The data fit linear lines as expected from Eq. (5.8), with the following magnitudes for the attempt frequency and the energy barrier $\mathrm{E}_{\mathrm{a}} / \mathrm{k}: \mathrm{f}_{0}=9.2 \times 10^{11} \mathrm{~Hz}$ and $\mathrm{E}_{\mathrm{a}} / \mathrm{k} \approx 1085(45) \mathrm{K}$ for the coated NP and $\mathrm{f}_{0}=1.4 \times 10^{39} \mathrm{~Hz}$ and $\mathrm{E}_{\mathrm{a}} / \mathrm{k} \approx 18250(1585) \mathrm{K}$ for the uncoated NP. For the coated NP, the above results are in line with the theoretical estimates for AF NP and the observed values for other systems [Kilcoyne et al, 1995, Ibrahim et al, 1995 and Dickson et al, 1993]. However, for the uncoated NP, the above magnitudes of $\mathrm{f}_{0}$ and $\mathrm{E}_{\mathrm{a}} / \mathrm{k}_{\mathrm{B}}$ are too large and unphysical, leading us to conclude that the NA relation is not valid for the uncoated NP as expected.

The differences observed in the variation of $\ln \mathrm{f} v \mathrm{vs} .1 / \mathrm{T}_{\mathrm{B}}$ for coated and uncoated $\mathrm{NP}$ are interpreted in terms of the presence of interparticle interaction in the uncoated NP and its absence in the coated NP. In order to check the validity of the VF law, we plot $\ln f$ vs. $T_{B}$ with fitting curves in the VF law (Eq. (5.7)) shown in Fig. 5.14. In the theoretical fitting, we used the values of $\mathrm{E}_{\mathrm{a}} / \mathrm{k}=1083 \mathrm{~K}$ and $\mathrm{f}_{0}=9.2 \times 10^{11} \mathrm{~Hz}$ derived from the NA relation for the coated NP and from this fitting, we obtained $\mathrm{T}_{0}=162 \mathrm{~K}$ for the uncoated $\mathrm{NP}$ and $\mathrm{T}_{0}=0 \mathrm{~K}$ for the coated NP. Here, the values of $\mathrm{T}_{0}$ represents a measure of the interparticle interaction on magnetic relaxation and these magnitudes of $T_{B}$ are comparable to the difference in the values of blocking temperatures for uncoated and coated NP shown in Fig. 5.3 for DC measurements. The fit of the data to Eq. (5.5) is very good, except for a slight difference at $\mathrm{f}=9.28 \times 10^{9} \mathrm{~Hz}$ for the uncoated NP. This may be related to the fact that the VF law is valid only for weak interparticle interaction and at higher f, deviations from the VF law are expected for stronger interparticle interaction [Dormann et al, 1988 and 1996, Shtrikman et al, 1981, and Zhang et al, 1996]. 

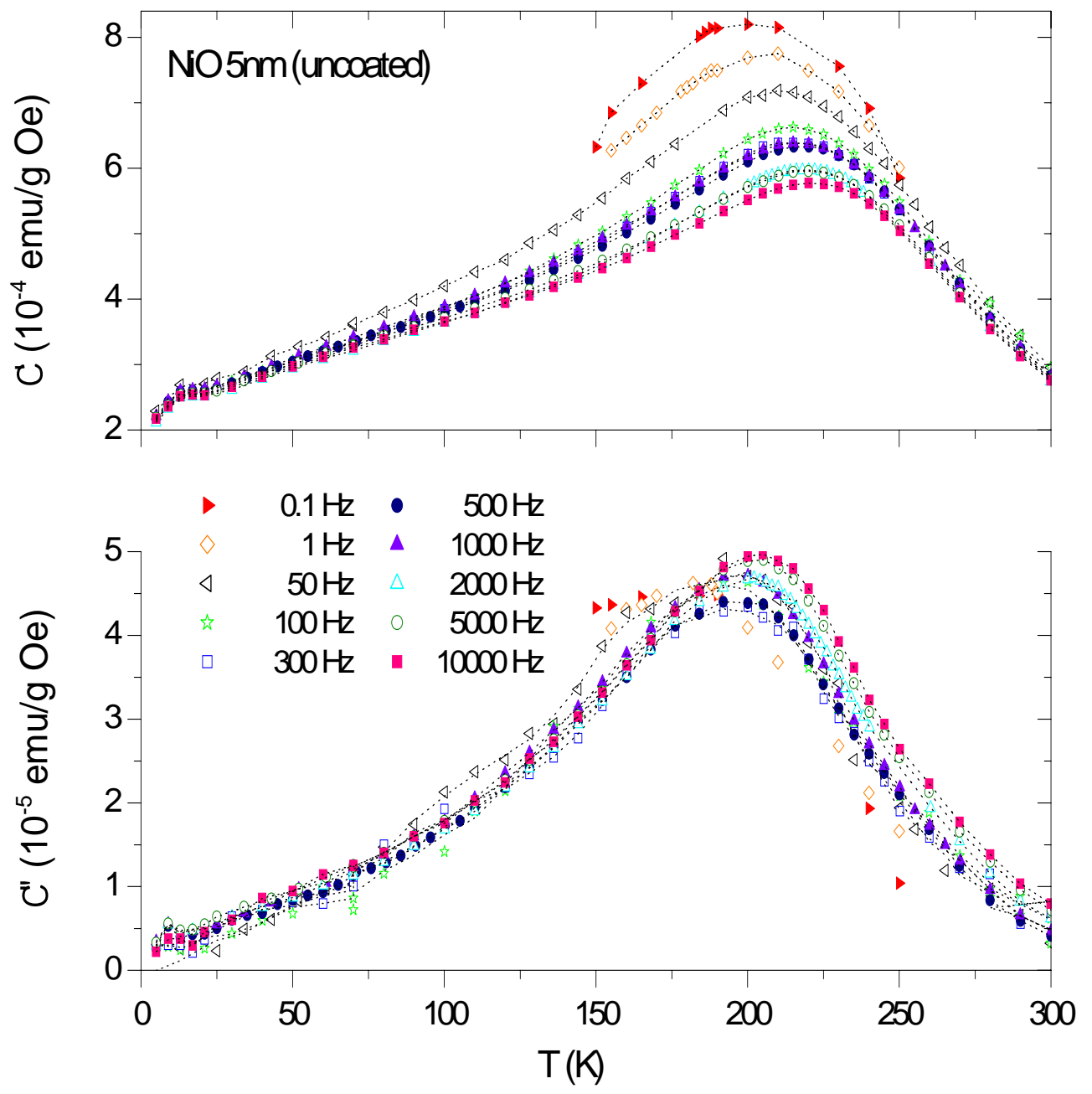

Fig. 5.11 Temperature variation of $\chi^{\prime}$ and $\chi^{\prime \prime}$ in uncoated NiO NP at different frequencies is shown. The lines through the points are for visual aid. 

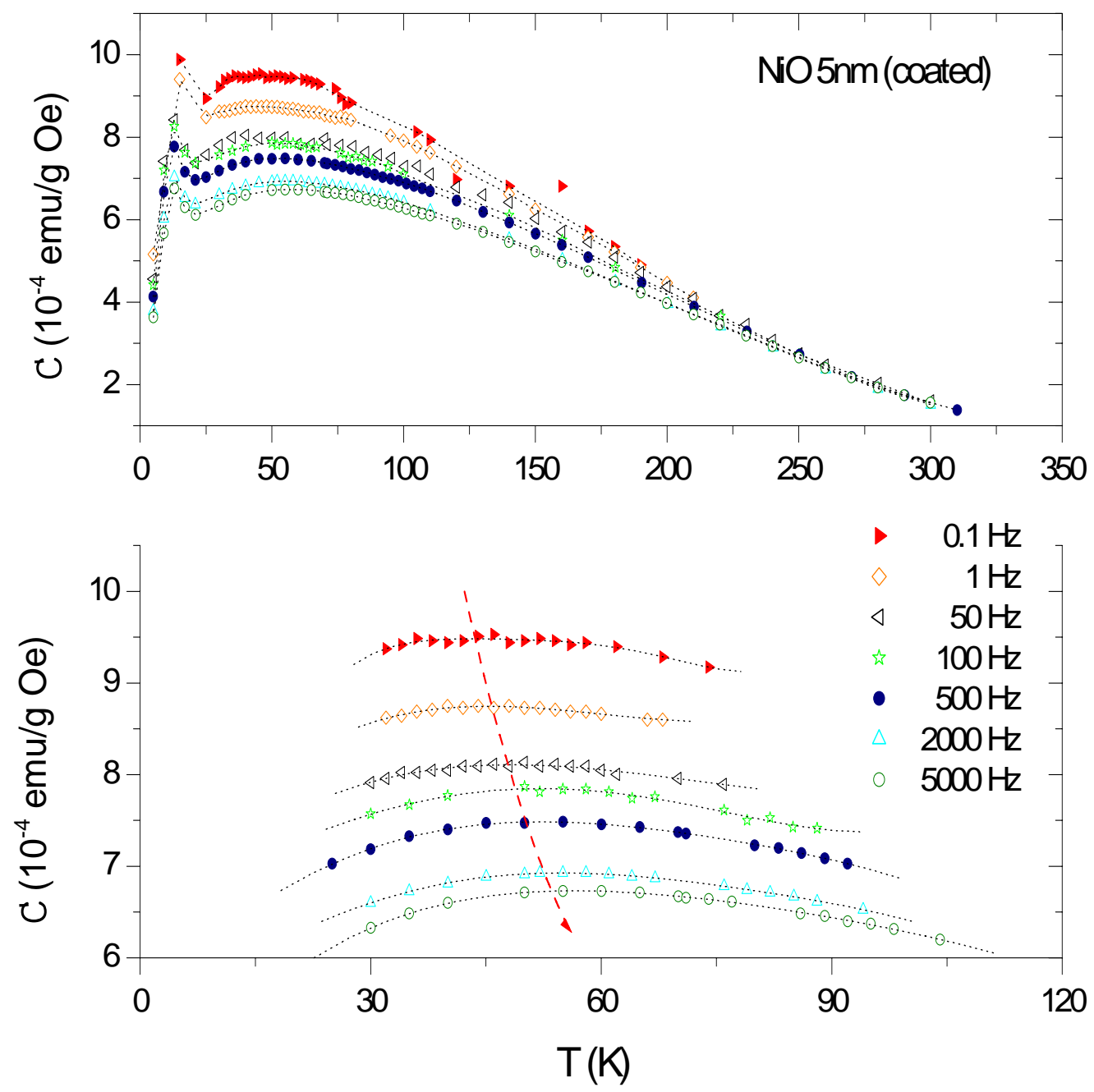

Fig. 5.12 Temperature dependence of $\chi^{\prime}$ vs. T for the coated NP. 


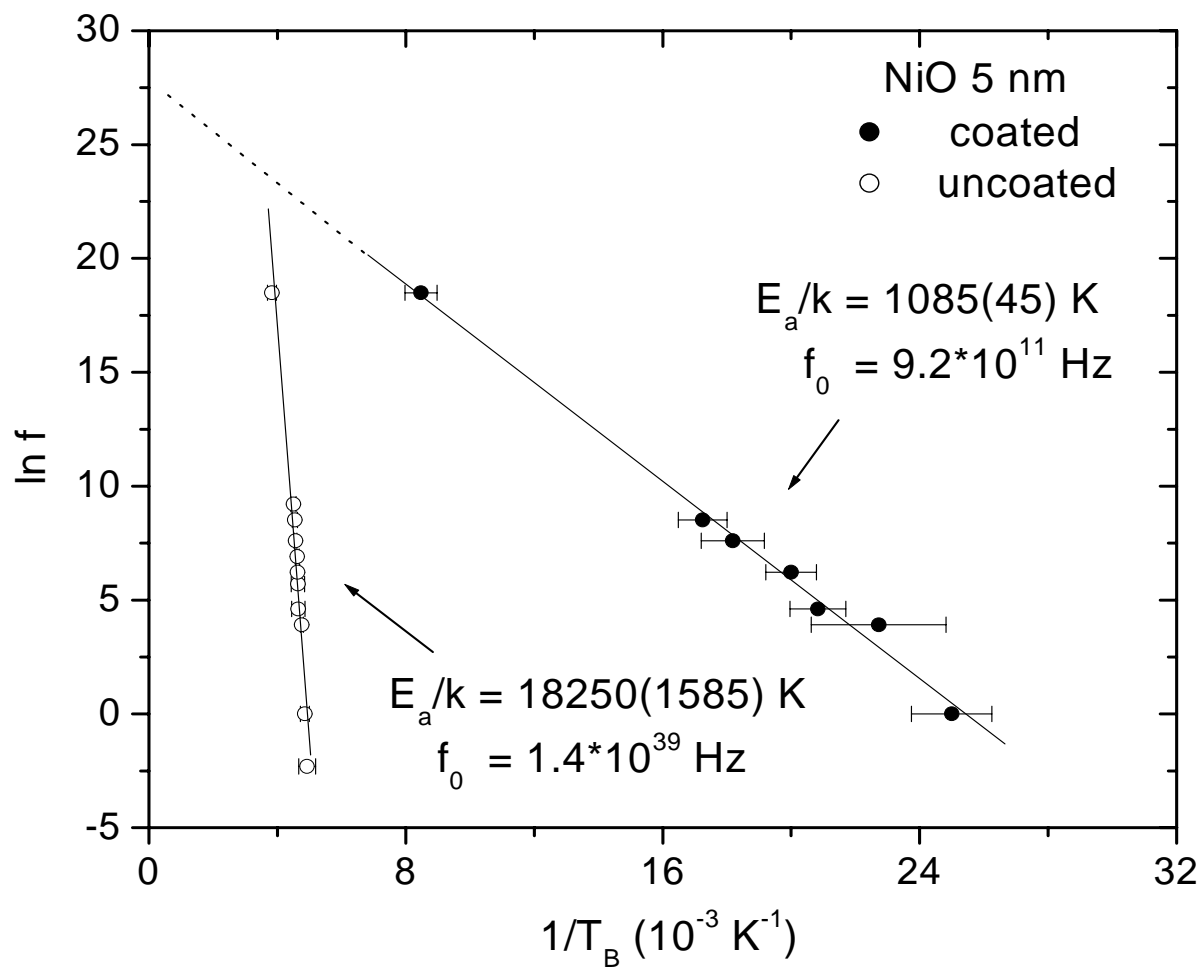

Fig. 5.13 Plot of $\ln \mathrm{f}$ against $1 / \mathrm{T}_{\mathrm{B}}$ for the coated and uncoated NiO NP. The solid lines are fits to Eq. (5.8) with parameters given in the plot. The size of error bars for the uncoated sample is within the size of the symbols. 
From Eq. (5.5), it also follows that

$$
\mathrm{T}_{\mathrm{B}}=\mathrm{T}_{0}+\left(\mathrm{E}_{\mathrm{a}} / \mathrm{k}\right) / \ln \left(\mathrm{f}_{0} / \mathrm{f}\right)
$$

Eq. (5.6) shows that interparticle interaction enhances the $T_{B}$ by $T_{0}$ so that the theoretical fits in Fig. (5.14) for uncoated NP are simply shifted by $T_{0}$. This enhancement of $T_{B}$ by interparticle interaction is in agreement with more explicit calculations for the dipolar interparticle interaction [Chantrell et al, 2000]. Another view of this enhancement of $T_{B}$ is that the interactions enhance correlation and so effectively increase the particle volume V [Allia et al, 2001, Vargas et al, 2005 and Denardin et al, 2002]. This enhances $T_{B}$ since $E_{a}=K V$, and hence $T_{B}$ is proportional to $V$,

In summary, comparing the changes in $\mathrm{T}_{\mathrm{B}}$ as a function of applied field $\mathrm{H}$ and measuring frequency $f_{m}$ in the coated and uncoated $5 \mathrm{~nm} \mathrm{NP}$ of $\mathrm{NiO}$, the effect of the presence of the interparticle interaction in the uncoated particles and its absence in the coated particles has been demonstrated. 


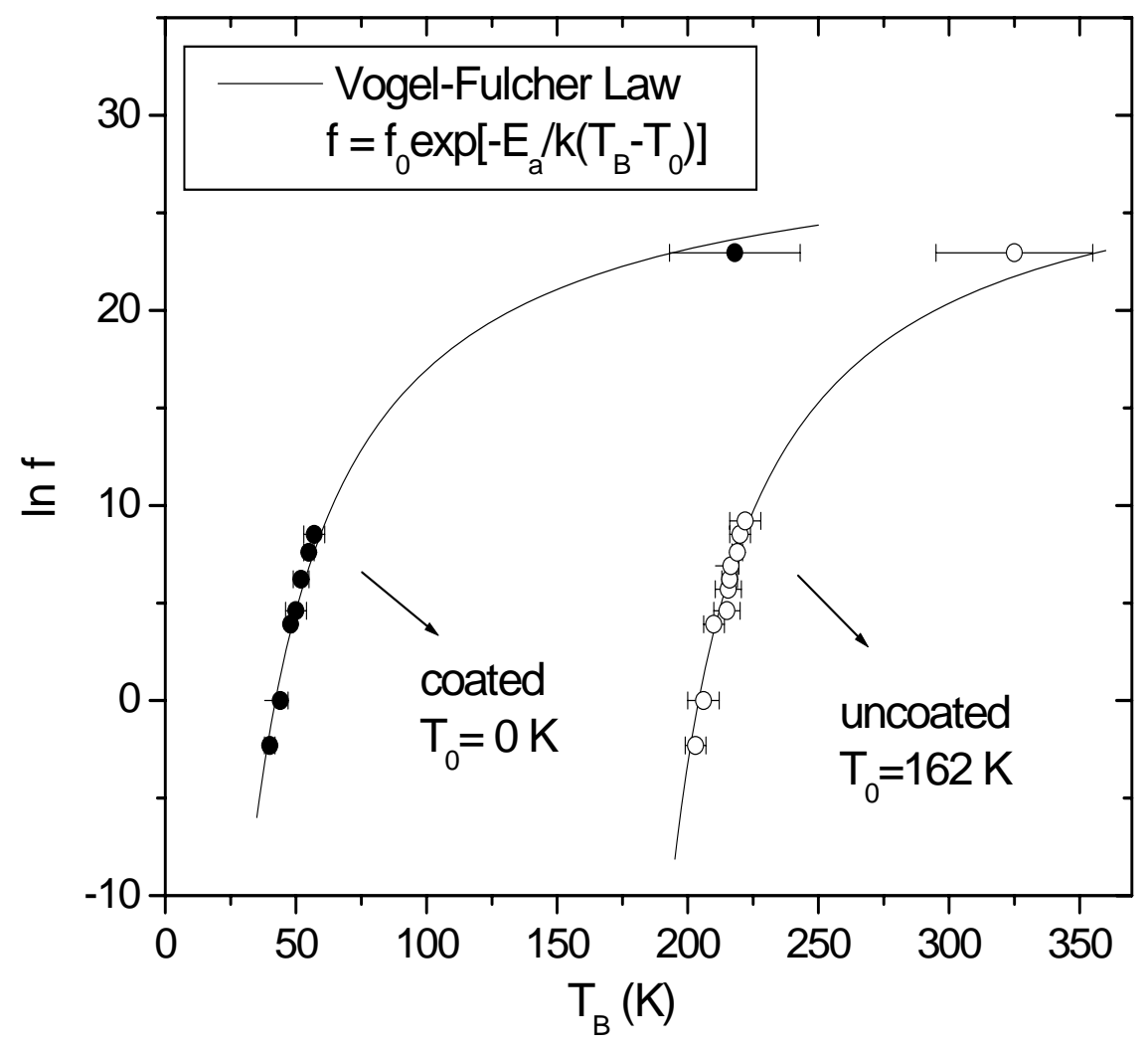

Fig. 5.14 Plot of $\ln f$ against $\mathrm{T}_{\mathrm{B}}$ for the coated and uncoated NiO $5 \mathrm{~nm}$ NP. The solid lines are fits to Eq. (5.5) with $\mathrm{T}_{0}=162 \mathrm{~K}$ for the uncoated and $\mathrm{T}_{0}=0 \mathrm{~K}$ for the coated $\mathrm{NiO} \mathrm{NP}$. The magnitudes of $E_{a} / k$ and $f_{0}$ used in the fit are the same as in Fig. 5.13. 


\section{CHAPTER VI}

\section{Size Dependence of the Magnetic Properties in NiO Nanoparticles}

\subsection{Introduction}

The structure and morphology of NiO-NP were investigated through the analysis of XRD and TEM in Chapter III. TEM studies show that the shapes of the NiO-NP change with increasing particle sizes. For the smaller particles, more of the particles are in the form of nanorods whereas for the larger particles produced by annealing temperatures, $\mathrm{T}_{\mathrm{a}} \geq 673 \mathrm{~K}$, nanorods change to particles with rounded morphology. Also there is a high degree of scatter in XRD data for smaller particle sizes due to the rod like morphology. Results of our magnetic studies on nominal $5 \mathrm{~nm} \mathrm{NiO} \mathrm{NP}$ [Seehra et al. 2004, 2005 and Shim et al. 2006] were described in chapter 4 where the differences between the magnetic properties of oleic-acid (OA) coated and uncoated particles were explained in terms of the interparticle interaction (negligible for coated particles).

In this chapter, I focus on the changes in the magnetic properties of the NiO-NP with increase in the nominal XRD size D from $5 \mathrm{~nm}$ to about $20 \mathrm{~nm}$. To investigate the effect of interparticle interaction, for all the particles, comparisons between the magnetic properties of the OA coated and the uncoated particles are provided. The variations of the magnetization $\mathrm{M}$ with temperature $\mathrm{T}(0 \mathrm{~K}$ to $370 \mathrm{~K})$ for the uncoated and coated samples, are measured by employing the ZFC and FC modes. From these measurements, blocking temperature $T_{B}$ is determined for each particle size. Fits of the magnetization $M$ vs. applied $\mathrm{H}$ at different temperatures $\mathrm{T}>\mathrm{T}_{\mathrm{B}}$ to the modified Langevin function (Eq. (4.16)) is used to determine $\mu_{\mathrm{P}}$ as a function of size D. Analysis of these data shows that the fraction $f$ of spins in the surface layer of the NP, which contribute a moment of $2.2 \mu_{\mathrm{B}}$ to $\mu_{\mathrm{P}}$ changes with $\mathrm{D}$. It is observed that this fraction $\mathrm{f}$ varies as $1 / \mathrm{D}$ reaching nearly $100 \%$ for the $5 \mathrm{~nm}$ particles. The Néel temperature $\mathrm{T}_{\mathrm{N}}$ decreases rapidly for $\mathrm{D}<10 \mathrm{~nm}$. 


\subsection{Magnetization vs. Temperature}

The measurements of $\mathrm{M}$ vs. T were carried out under the zero-field-cooled (ZFC) and field-cooled (FC) modes following the same procedure as in section 5.2. For ZFC, the sample is cooled to $5 \mathrm{~K}$ in zero magnetic field, a magnetic field $\mathrm{H}=100 \mathrm{Oe}$ is then applied at $5 \mathrm{~K}$ followed by measuring $\mathrm{M}$ with increasing $\mathrm{T}$ after stabilizing the temperature at each temperature till $\mathrm{T}=370 \mathrm{~K}$ is reached. For the $\mathrm{FC}$ case, the data are then taken with decreasing $\mathrm{T}$ in a similar fashion. The data so taken for different samples are shown in Fig. 6.1 for the uncoated particles and in Fig. 6.2 for the coated particles. Note that the temperatures $T_{P}$ at which the magnetic susceptibility $\chi(=M / H)$ peaks for the $\mathrm{ZFC}$ cases are also indicated. $\mathrm{T}_{\mathrm{P}}$ represents the average blocking temperature for each particle. The temperatures at which the ZFC and FC data bifurcates are generally higher than $T_{P}$, most likely due to the wide particle size distribution, especially for large $\mathrm{D}$. The plot of $T_{P}$ vs. particle size $D$ is shown in Fig. 6.3 for both the coated and the uncoated particles. A noteworthy feature of this plot is that for $\mathrm{D}<10 \mathrm{~nm}, \mathrm{~T}_{\mathrm{P}}$ for the uncoated particles continues to increase with decrease in $\mathrm{D}$ whereas the trend for the coated particles is just the opposite. Note that $T_{P}$ for non-interacting particles is expected to be proportional to the particle volume $\mathrm{V}$ (Eq. (4.8)) in line with the observations for the coated particles. The results for the uncoated particles can be interpreted in terms of the interparticle interaction. The reason is explained as below.

Following the discussion on the magnetic relaxation in the $5 \mathrm{~nm} \mathrm{NiO}$ nanorods [Shim et al. 2006] in Chapter $\mathrm{V}$, the relaxation rate $\mathrm{f}$ for the magnetization for $\mathrm{T}>\mathrm{T}_{\mathrm{P}}$ follows the Vogel-Fulcher law [Shtrikman et al. 1981, Dormann et al.1988, and Zhang et al. 1996]. The second term on the right-hand side of Eq. (5.8) represents the blocking temperature for non-interacting NP's $\left(T_{0}=0\right)$ at the measuring frequency $f_{m}$. Using the data of the variation of $T_{P}$ with measuring frequency f from $\mathrm{f}=0.1 \mathrm{~Hz}$ to $10^{4} \mathrm{~Hz}$ in the 5 $\mathrm{nm} \mathrm{NiO}$ nanorods, it was shown that $\mathrm{T}_{0}=162(0) \mathrm{K}$ for the uncoated (coated) nanorods with $\mathrm{E}_{\mathrm{a}} / \mathrm{k}=1083 \mathrm{~K}$ and $\mathrm{f}_{0}=9.2 \times 10^{11} \mathrm{~Hz}$ [Shim et al. 2006]. The relevance of the above results for the data presented here is that it also provides an explanation for the data of Fig. 6.3 in that the enhanced $T_{P}$ for the uncoated particles is simply due to the interparticle interaction (Note that in general $T_{P}=\beta T_{B}$ where $\beta$ takes into account the particle size distribution, with $\beta=1.5-2.0$ depending on the distribution [Gittleman et al. 
1974 and El-Hilo et al. 1992]). Since $E_{a}=K V$ where $K$ is the anisotropy constant and V is the volume of the particles, the increase in $T_{B}$ with increase in size $D$ is expected as observed in Fig. 5.3 for the coated particles. Another view of this enhancement of $\mathrm{T}_{\mathrm{B}}$ by interparticle dipolar interaction is that the interactions enhance correlations and this effectively increases the particle volume V [Chantrell et al. 2000, Allia et al. 2001, Denardin et al. 2002, and Vargas et al. 2005]. The question of why the effects of interparticle interaction become negligible for larger particles is addressed later.

In a recent paper on the uncoated $\mathrm{NiO}$ nanoparticles with sizes $\mathrm{D}=5.1,6.2$ and $8.5 \mathrm{~nm}$, Tiwari and Rajeev [Tiwari et al. 2005] reported somewhat similar behavior of the decreases of $T_{P}$ with increase in particle size. Although their particles were prepared by the similar sol-gel method, TEM studies were not included in this paper so that it is not possible to compare the morphological features. They argue that the $T_{P}$ in their samples really represents spin-glass freezing and not the effect of interparticle dipole-dipole interaction. Their argument is partly based on their assumption that $\mu_{\mathrm{P}} \approx 100 \mu_{\mathrm{B}}$ (although no Langevin-type analysis of the data to determine $\mu_{\mathrm{P}}$ was presented). On the other hand, our analysis presented here shows that $\mu_{\mathrm{P}} \geq 10^{3} \mu_{\mathrm{B}}$ for $\mathrm{D} \approx 5 \mathrm{~nm}$, making the interparticle interaction more important. Spin-glass freezing of the surface spins has been observed in NP of ferrihydrite [Punnoose et al. 2005] and $\gamma-\mathrm{Fe}_{2} \mathrm{O}_{3}$ [Martínez et al. 1998 and Koksharov et al 2000] but in these cases, the spin-glass freezing temperature $T_{S}$ is considerably lower than $T_{\mathrm{P}}$. Analysis of our AC susceptibility data for the coated and uncoated $\mathrm{NiO}$ nanoparticles also shows that in coated particles, the interparticle interaction is negligible whereas in uncoated $5 \mathrm{~nm}$ particles, an effective interparticle interaction temperature $\mathrm{T}_{0} \approx 162 \mathrm{~K}$ in Eq. (5.6) is needed to fit the relaxation data [Shim et al. 2006]. Further evidence that OA coating essentially eliminates the interaction is evident from the increase of $T_{P}$ with size $D$ for the coated particles, as expected for superparamagnetic particles (Fig.6.3). 

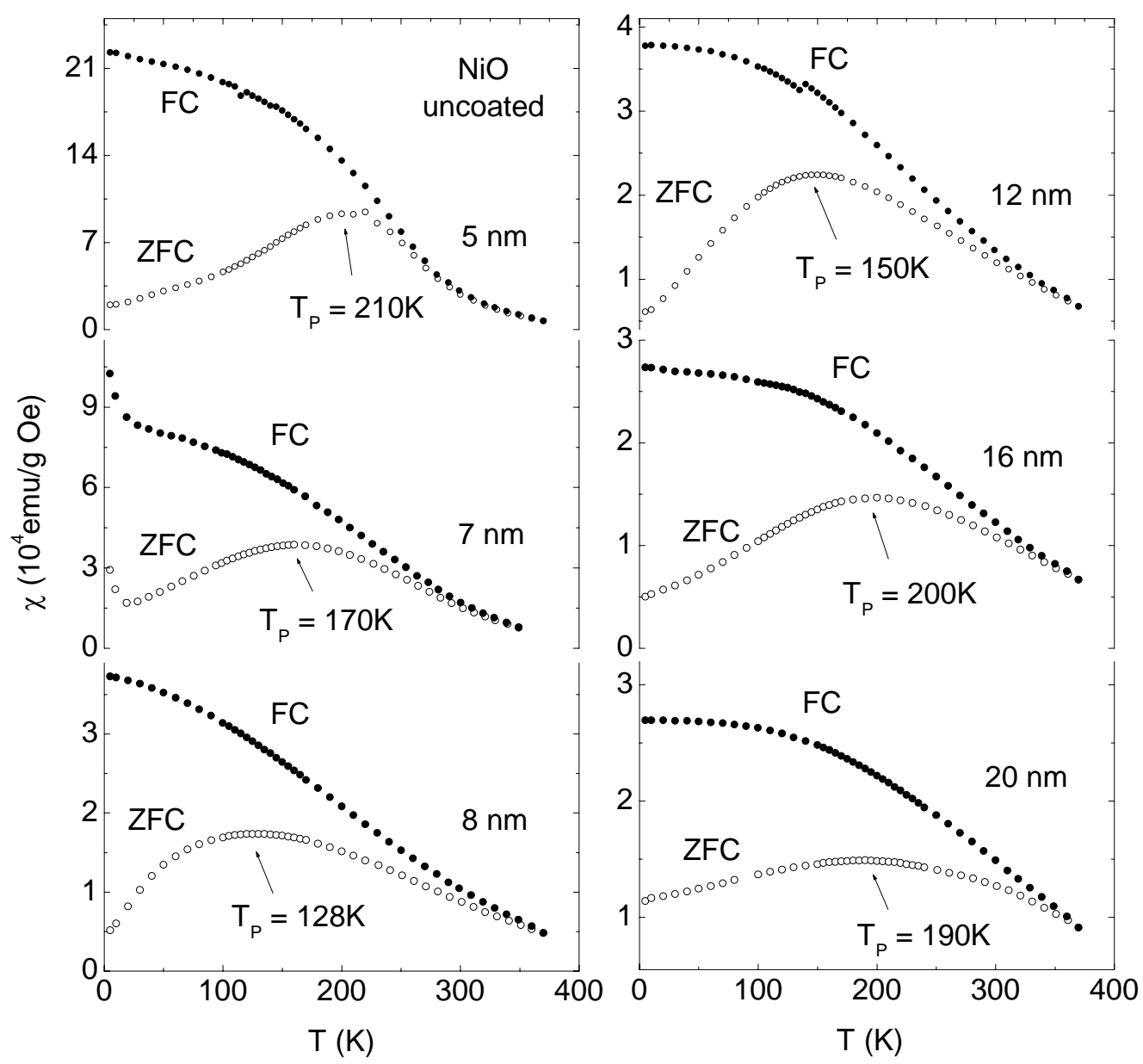

Fig. 6.1 Temperature variation of the magnetic susceptibility $\chi$ of the uncoated NiO-NP measured in $\mathrm{H}=100 \mathrm{Oe}\left(10^{4} \mathrm{Oe}=1\right.$ Tesla $)$ for the FC and ZFC modes. The arrows indicate the location of $\mathrm{T}_{\mathrm{P}}$, the temperatures at which $\chi(\mathrm{ZFC})$ peaks for each case. 


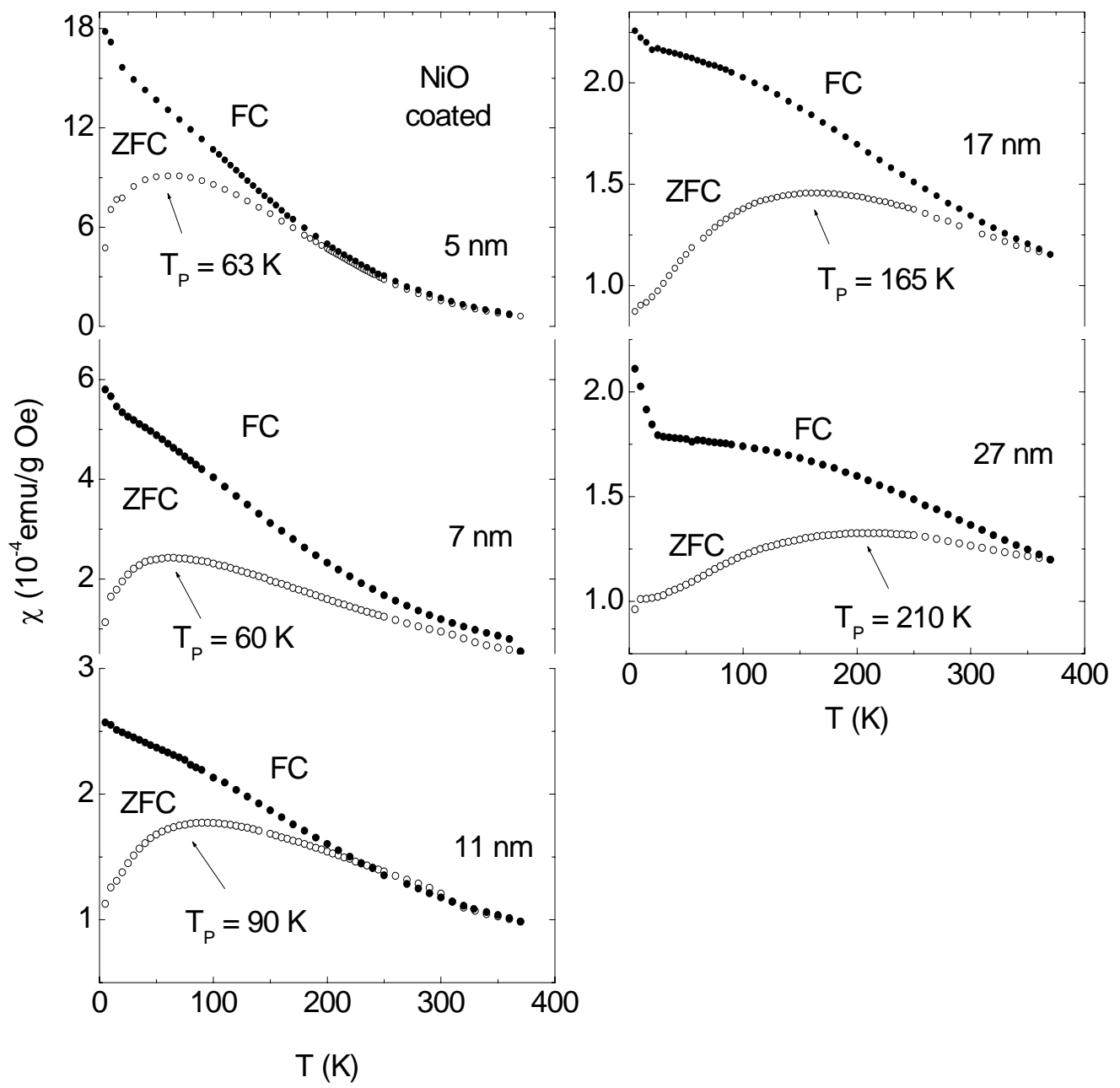

Fig. 6.2 Same as Fig. 6.1 except for the OA coated NiO-NP. 


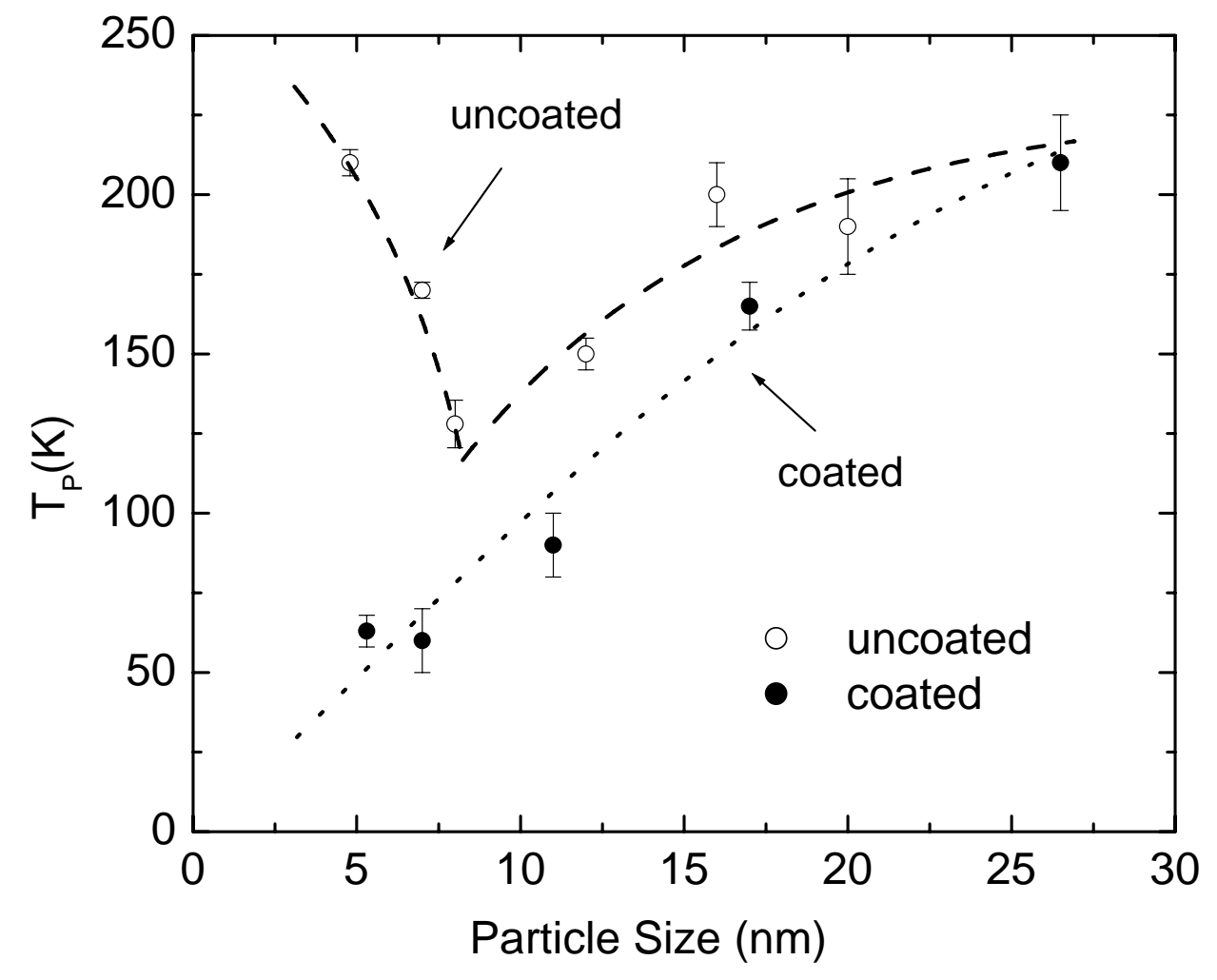

Fig. 6.3 Variation of $T_{P}$ with particle size for the uncoated and coated NiO-NP. The dotted lines through the points are a guide to eye. 


\subsection{Magnetization vs. Magnetic Field}

For $\mathrm{T}>\mathrm{T}_{\mathrm{P}}$ where the particles are expected to be in the superparamagnetic (SPM) state, The magnetization $\mathrm{M}$ is measured in field $\mathrm{H}$ up to $55 \mathrm{kOe}$ in the temperature range of 230 (200) to 370 (300) K for uncoated (coated) particles. These plots of M vs. H are shown in Fig. 6.4 for the uncoated particles and in Fig. 6.5 for the coated particles. It is evident that $\mathrm{M}$ does not saturate even at $\mathrm{H}=55 \mathrm{kOe}$ and there is a high field magnetic susceptibility $\chi_{a}$. For the larger particles with wider size distributions $(D=17 \mathrm{~nm}$ and 27 $\mathrm{nm}$ coated), a small remanence at $\mathrm{H}=0$ was observed probably because the larger particles are still unblocked at these temperatures as attested by the data in Fig. 6.2. This remanence was subtracted out in fitting the data to the Langevin function [Resnick et al. 2006].

In order to figure out the parameters $\mathrm{M}_{0}, \mu_{\mathrm{P}}$, and $\chi_{\mathrm{a}}$ from the Langevin function fitting, first in the experimental data measured in each temperature in Fig. 6.4 and Fig. 6.5, $\chi_{\mathrm{a}}$ and $\mathrm{M}_{0}$ are estimated from measuring the slope of linear part in high field of $\mathrm{M}$ vs. $\mathrm{H}$ plot and reading the value of intersection point with $\mathrm{y}$-axis in the plot of $\left(\mathrm{M}-\chi_{\mathrm{a}} \mathrm{H}\right) \mathrm{vs}$. $1 / \mathrm{H}$ as $\mathrm{H}$ goes to infinity respectively. These estimated values of $\chi_{\mathrm{a}}$ and $\mathrm{M}_{0}$ have temperature dependence as shown in Fig. 6.6 and Fig. 6.7. For the smaller particles, larger slopes are observed.

Using these estimated parameters, $\chi_{\mathrm{a}}$ and $\mathrm{M}_{0}$, the variation of $\mathrm{M}$ vs. $\mathrm{H}$ for $\mathrm{T}>\mathrm{T}_{\mathrm{P}}$ is fitted to the modified Langevin function in a manner similar to the one used in earlier studies of doped ferrihydrite [Punnoose et al, 2004] and ferritin NP [Makhlouf et al, 1997]. The plots of $\left(\mathrm{M}-\chi_{\mathrm{a}} \mathrm{H}\right) / \mathrm{M}_{0}$ against $\mathrm{H} / \mathrm{T}$ are shown in Fig. 6.8 for the uncoated and coated particles with the solid lines as the fits with the $\mu_{\mathrm{P}}$ as shown in the figures. To see whether there is some temperature dependence to $\mu_{\mathrm{P}}$, the variation of $\mu_{\mathrm{P}}$ with temperature is estimated by fitting the data in Fig. 6.4 and Fig. 6.5 using non-linear fitting in program ORGIN at each temperature. The magnitudes of $\mu_{P}$ vs. temperature so determined are plotted in Fig. 6.9 for both the uncoated and coated particles. For the smaller particles, there is no significant temperature dependence to $\mu_{\mathrm{P}}$ as the fits in Fig. 6.8. However for the larger particles, $\mu_{P}$ increase until one temperature point around 280 (350) for coated (uncoated) samples and after that point, $\mu_{P}$ decreases. 
Recently, the topic of how $\mu_{\mathrm{P}}$ in AF-NP varies with temperature has received considerable attention. Partly based on the experimental results of Seehra et al. [2000] in ferrihydrite nanoparticles where $\mu_{\mathrm{P}}$ was found to increase with temperature, Mørup et al [2004] proposed a theory which predicted increase of $\mu_{\mathrm{P}}$ with temperature in AF-NP. However, Silva et al. [2005] have recently argued that a wide particle-size distribution can mimic $\mu_{\mathrm{P}}$ increasing with temperature. Our studies also show that for larger particles (e.g. $\mathrm{D} \approx 20 \mathrm{~nm}$ ) with wider size distribution, $\mu_{\mathrm{P}}$ apparently shows an increase with increase in temperature. For smaller particles, $\mu_{\mathrm{P}}$ is essentially temperature independent. In our analysis, we have not taken into account the particle size distribution since the size distribution in most of our cases does not follow any simple distribution function such as lognormal or Gaussian. Nevertheless for the smaller particles, our data show that $\mu_{\mathrm{P}}$ is temperature independent or decreases slowly with increase in temperature. 


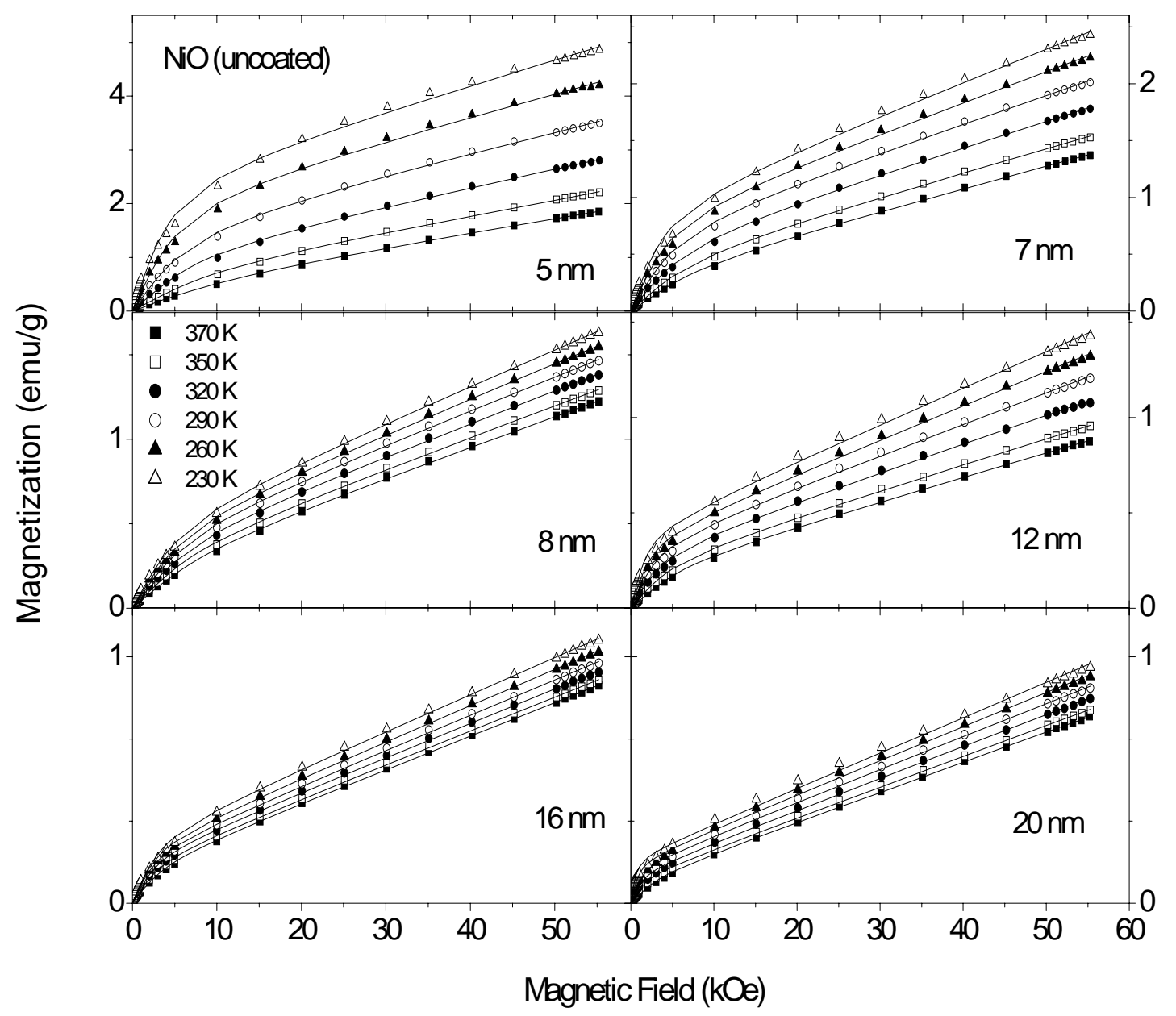

Fig. 6.4 Plots of measured magnetization $\mathrm{M}$ vs. applied field $\mathrm{H}\left(10^{4} \mathrm{Oe}=1\right.$ Tesla $)$ for the uncoated NiO-NP at several temperatures above $T_{P}$. The lines joining the points are for visual aid. 


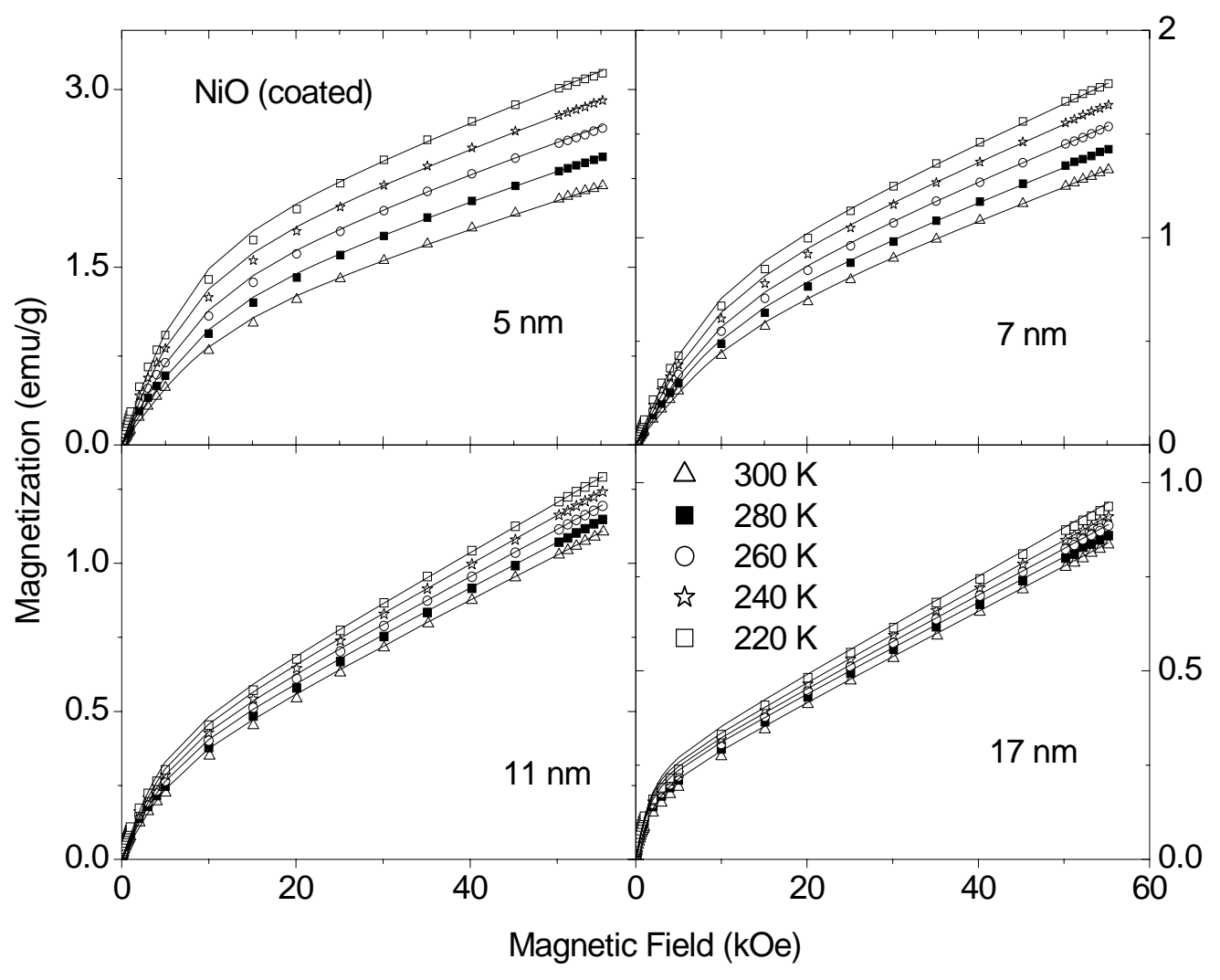

Fig. 6.5 Same as in Fig. 5.4 except the data are for the OA coated NiO-NP. 


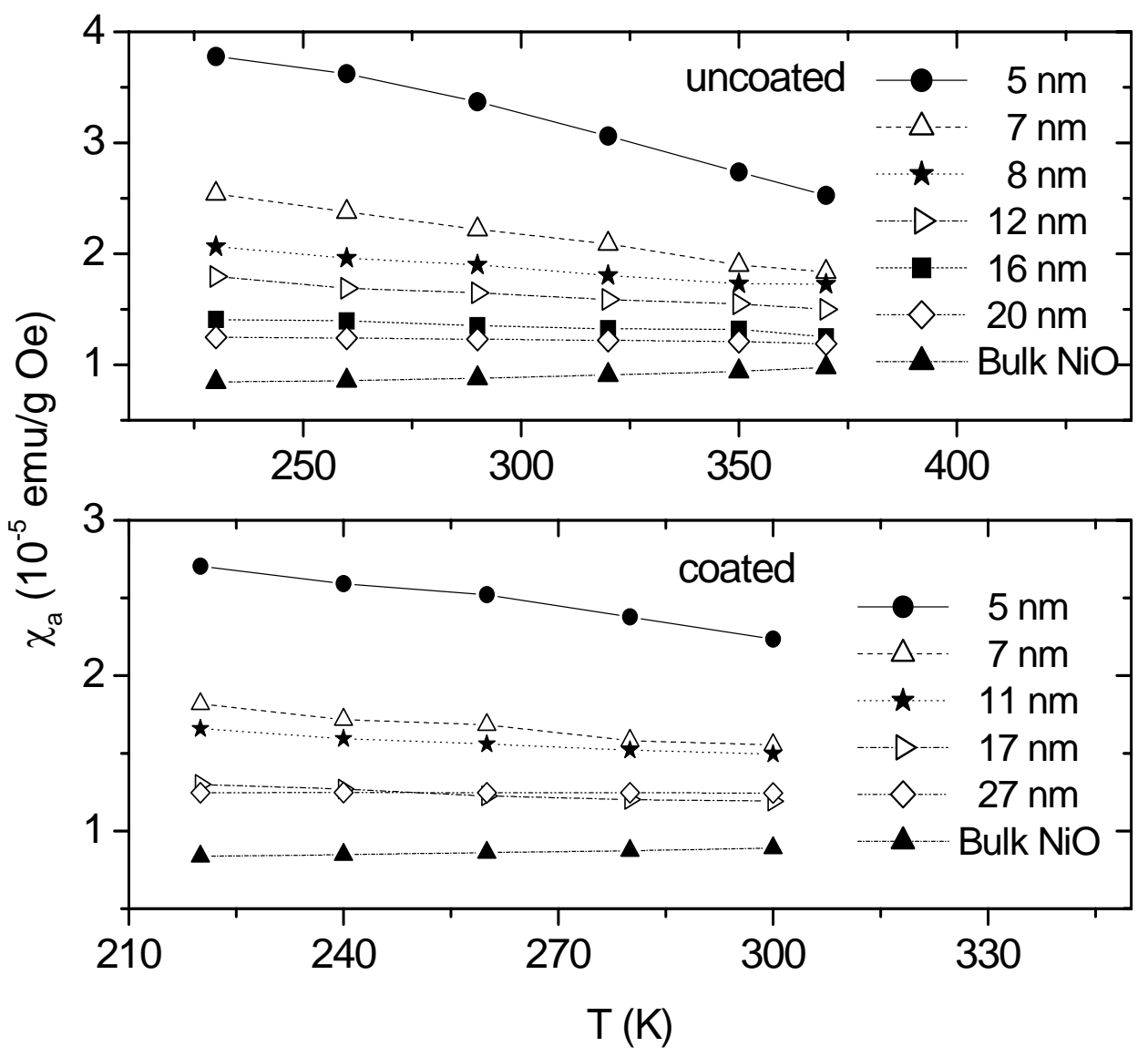

Fig. 6.6 Plots of $\chi_{a}$ vs. T show the linear decrease of $\chi_{a}$ with temperature. 


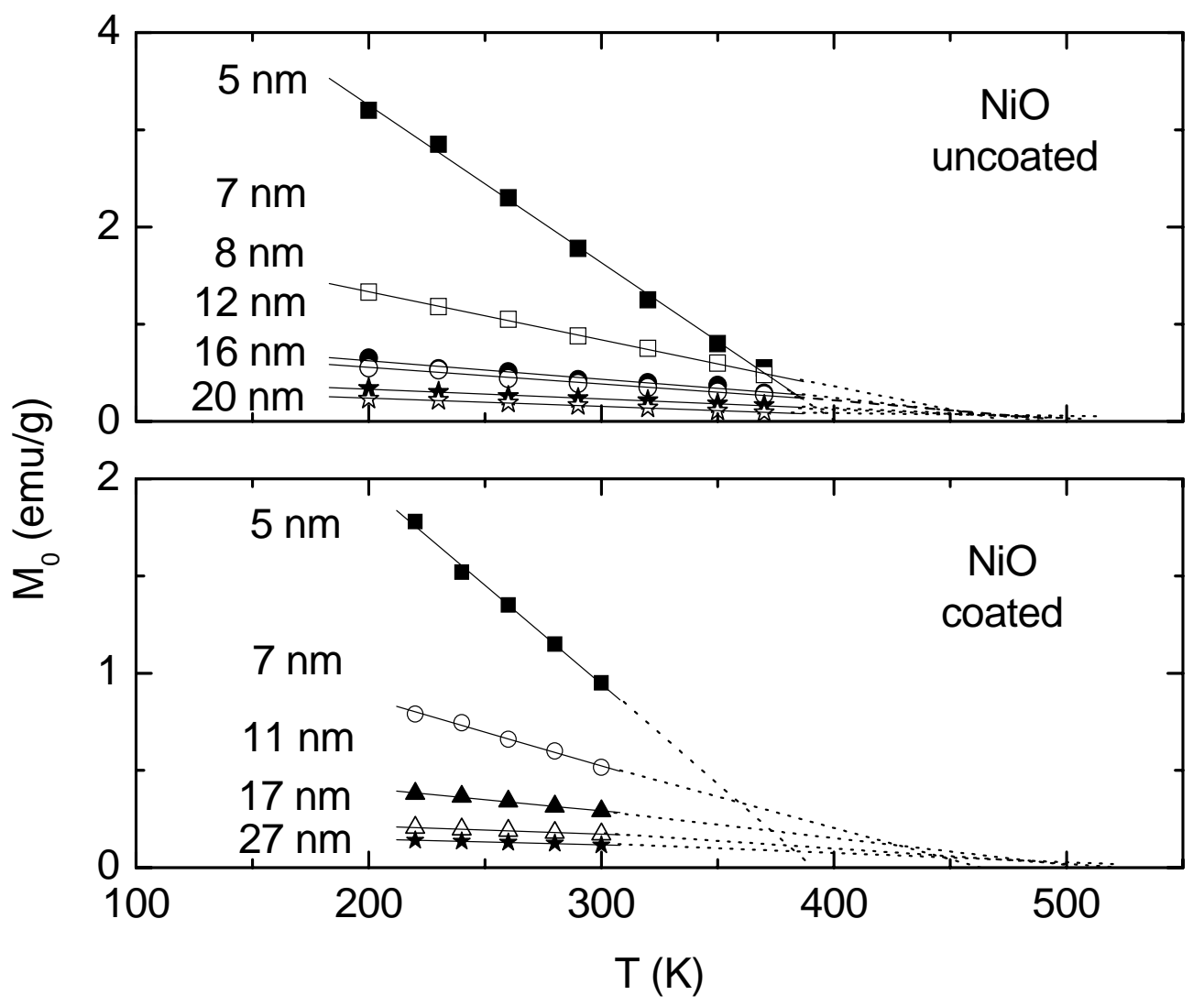

Fig. 6.7 Temperature dependence of $\mathrm{M}_{0}$ (Eq. (4.16)) for the uncoated and coated NiONP for $T>T_{P}$. The solid lines through the data points represent linear variations whereas the dotted lines are extrapolations to $\mathrm{M}_{0} \rightarrow$ zero to estimate the Néel temperature $\mathrm{T}_{\mathrm{N}}$. 

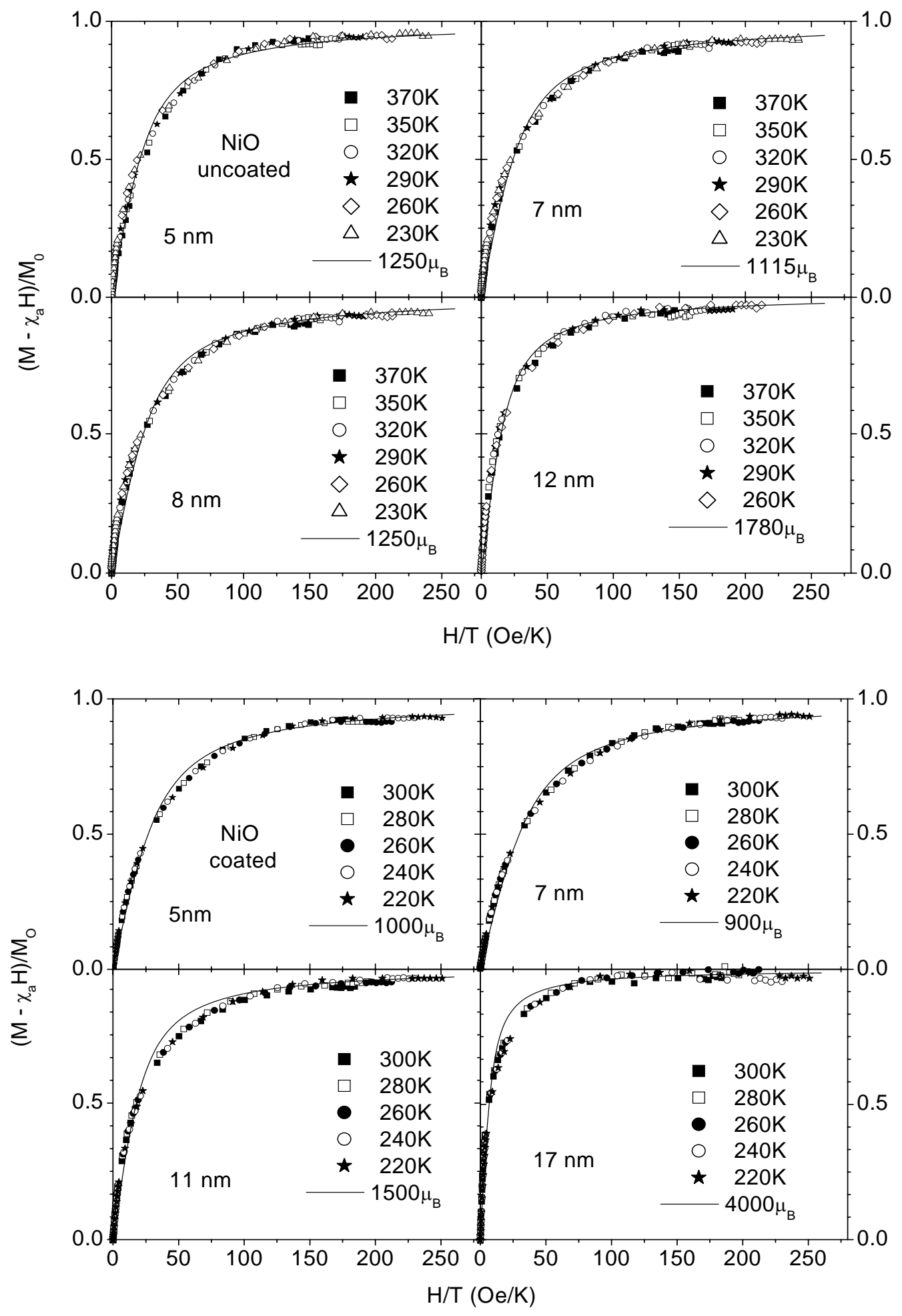

Fig. 6.8 Plots of $\left(\mathrm{M}-\chi_{\mathrm{a}} \mathrm{H}\right) / \mathrm{M}_{0}$ against $\mathrm{H} / \mathrm{T}$ for selected coated and uncoated NiO-NP. The solid lines are fits to Eq. (4.16) with $\mu_{\mathrm{P}}$ (magnetic moment/particle) values shown on the figures. 


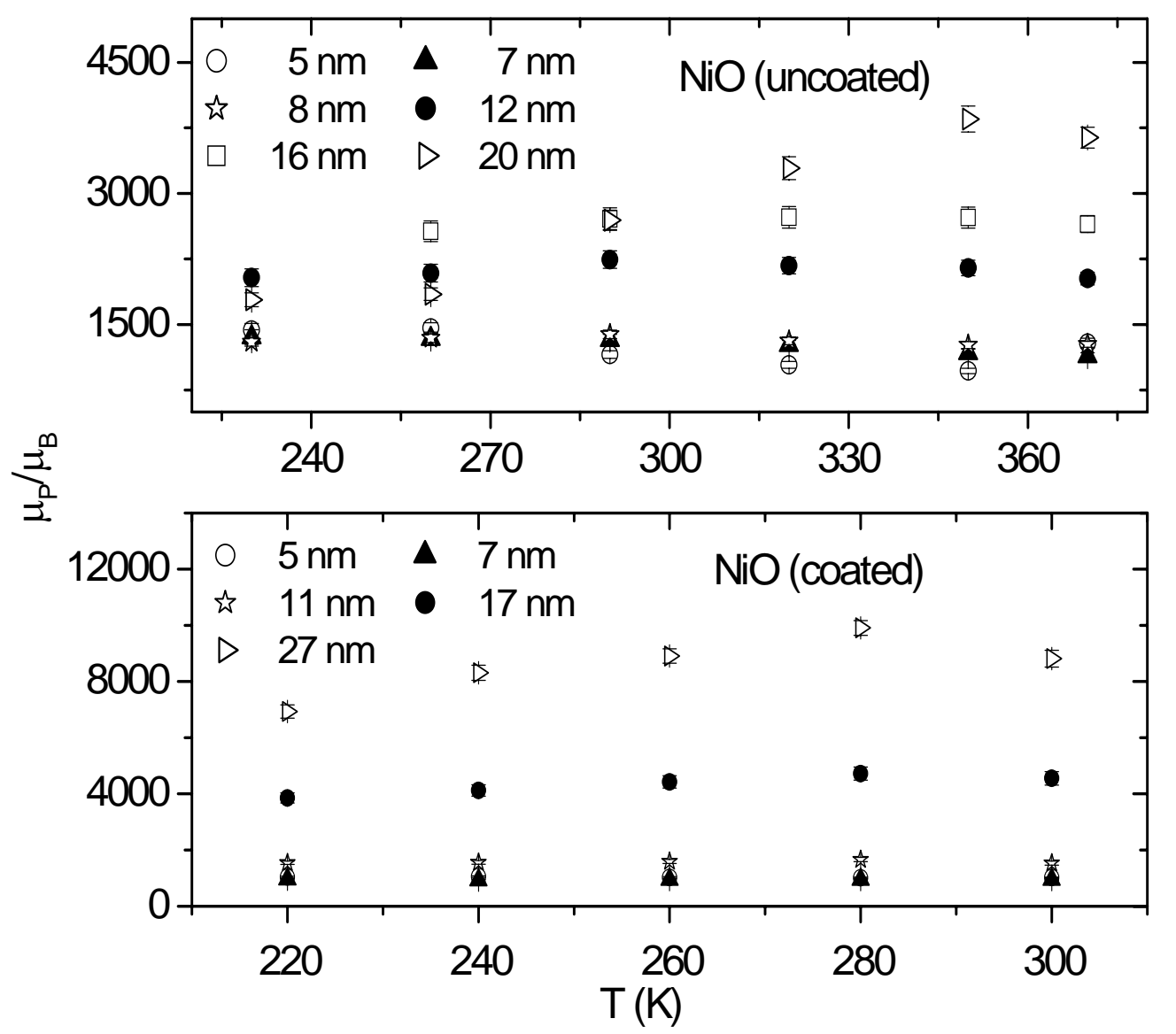

Fig. $6.9 \mu_{\mathrm{P}}$ determined at different temperatures by fitting the data of $\mathrm{M}$ vs. $\mathrm{H}$ to Eq. (4.16). For the smaller coated and uncoated $\mathrm{NiO}-\mathrm{NP}, \mu_{\mathrm{P}}$ so determined is essentially temperature independent. 


\subsection{Size dependence of $\mu_{P}$}

The variation of $\mu_{\mathrm{P}}$ with size $\mathrm{D}$ is addressed next. Néel suggested that $\mu_{\mathrm{P}}$ depends on the total number of atoms $n_{D}$, the crystal structure and particle morphology [Richardson et al. 1956, 1991 and Neel, 1961]. This model leads to $\mu_{P}=p \mu \mu_{B}$ where $p$ is the difference in the numbers of atoms in the two sublattices $A$ and $B$ of the antiferromagnet $\left(\mathrm{p}=\mathrm{n}_{\mathrm{A}}-\mathrm{n}_{\mathrm{B}}\right.$ and $\left.\mathrm{n}_{\mathrm{D}}=\mathrm{n}_{\mathrm{A}}+\mathrm{n}_{\mathrm{B}}\right)$ and $\mu_{\mathrm{B}}$ is Bohr magneton. How the atoms on the surface of the NP are arranged determines the dependence of $\mathrm{p}$ on $\mathrm{n}_{\mathrm{D}}$. For random ordering $\mathrm{p}=\mathrm{n}_{\mathrm{D}}^{1 / 2}$ but $\mathrm{p}=\mathrm{n}_{\mathrm{D}}{ }^{2 / 3}$ if surface atoms all belong to the same sublattice. Of course dependence of $\mu_{P}$ on size $D$ comes through $n_{D}$, the number of magnetic atoms per particle. In the case of $\mathrm{NiO}$, we consider $\mathrm{Ni}^{2+}$ ions on a FCC lattice with lattice constant of $4.1795 \AA$ to determine $n_{D}$ for each size $D$ assuming spherical particles of diameter $D$. Using the above information, we calculate $\mathrm{n}_{\mathrm{D}}=3,586$ for $\mathrm{D} \approx 5 \mathrm{~nm}$. This $\mathrm{n}_{\mathrm{D}}$ yields $\mu_{\mathrm{P}}=$ $515 \mu_{\mathrm{B}}$ for $\mathrm{p}=\mathrm{n}_{\mathrm{D}}^{2 / 3}$ and $\mu_{\mathrm{P}}=132 \mu_{\mathrm{B}}$ for $\mathrm{p}=\mathrm{n}_{\mathrm{D}}{ }^{1 / 2}$. These magnitudes are considerably smaller than the experimental value of $\mu_{\mathrm{P}} \approx 1250 \mu_{\mathrm{B}}$. Clearly this analysis does not describe the experimental result satisfactorily, a situation also noted in earlier studies [Makhlouf et al. 1997].

Assuming a core - shell model where a fraction $\mathrm{f}=\mathrm{n}_{\mathrm{C}} / \mathrm{n}_{\mathrm{A}}$ of the $\mathrm{Ni}^{2+}$ spins on the surface layer (shell) contribute to $\mu_{\mathrm{P}}$ and the spins in the core are antiferromagnetically ordered, we can calculate f by comparing the experimental $\mu_{\mathrm{P}}$ with the calculations (see Table 5.I). This fraction $\mathrm{f}$ is plotted as a function of $\mathrm{D}$ in Fig. 6.11, with the dashed line representing the $1 / \mathrm{D}$ variation ( $\ell n \mathrm{f}$ vs. $\ell n \mathrm{D}$ plot yielded the slope of negative one). There is a slight departure for this variation for the $\mathrm{D}=5 \mathrm{~nm}$ particles in such a way that not only all the spins in the top surface layer are contributors to $\mu_{\mathrm{P}}$ for this size, but some additional spins perhaps in the second adjacent layer at the surface are also contributing to $\mu_{\mathrm{P}}$ (sizes $\mathrm{D}$ for which is $\mu_{\mathrm{P}}$ temperature dependent in Fig. 5.9 are not included in Fig. 6.11). The model that emerges from this analysis is that with decrease in $\mathrm{D}$, a larger fraction of surface spins contribute to $\mu_{\mathrm{P}}$ as a result of their decoupling from the core spins. This model is physically appealing since surface spins have lower coordination and hence weaker exchange coupling to the spins in the core. Therefore it is likely that for D $<5 \mathrm{~nm}$, the long range AF order will eventually break down as D is decreased. In a recent work, Tang et al. [Tang et al. 2003] in antiferromagnetic $\mathrm{CoO}$ layers have reached a 
similar conclusion in that layers with thickness $\approx 1 \mathrm{~nm}$ were found to have $\mathrm{T}_{\mathrm{N}}$ approaching $0 \mathrm{~K}$.

From the comparison shown in Fig. 6.3, it is evident that the effect of interparticle interaction decreases with increase in size D. The interparticle dipolar interaction between two particles varies approximately as $\mu_{\mathrm{P}}{ }^{2} \mathrm{D}^{3}$. From Table 6.1 , although $\mu_{\mathrm{P}}$ increases by a factor about 3 from $D=5 \mathrm{~nm}$ to $\mathrm{D}=20 \mathrm{~nm}$, the magnitude of $\mu_{\mathrm{P}}{ }^{2} / \mathrm{D}^{3}$ decreases by a factor of $9 / 64$. In addition, the fraction $n_{C} / n_{D}$ goes down as $D$ increases approaching nearly zero for the largest particles. The effect of these factors is to lower the effects of interparticle interaction for larger particles quite significantly as observed experimentally.

\subsection{Size dependence of $T_{N}$}

In the plot of $\mathrm{M}_{0}$ vs. $\mathrm{T}$ in Fig. 6.7, $\mathrm{M}_{0}$ decreases linearly with increasing temperature. To explain this temperature dependence of $M_{0}$, Makhlouf et al. [1997] suggested that $\mathrm{M}_{0}$ originates from surface moments and surface moments should vary linearly with $T_{N}-T$ near $T_{N}$. Thus, we can write this in the form [Seehra et al. 2000 and Punnoose et al. 2004],

$$
\mathrm{M}_{0}=\mathrm{M}^{*}\left[1-\left(\mathrm{T} / \mathrm{T}_{\mathrm{N}}\right)\right]
$$

where $\mathrm{M}^{*}$ is the magnitude of $\mathrm{M}_{0}$ extrapolated to $\mathrm{T}=0 \mathrm{~K}$. This can be used to determine $\mathrm{T}_{\mathrm{N}}$ for the NiO-NP by assuming that linear extrapolation to $\mathrm{M}_{0} \rightarrow 0$ yields $\mathrm{T}_{\mathrm{N}}$. $\mathrm{T}_{\mathrm{N}}$ 's so determined are plotted against particle size D in Fig. 6.12. This result shows that for $\mathrm{D}<$ $20 \mathrm{~nm}, \mathrm{~T}_{\mathrm{N}}$ begins to decrease slowly and for $\mathrm{D}<10 \mathrm{~nm}$, it decreases rapidly from $\mathrm{T}_{\mathrm{N}}$ of bulk NiO. This is somewhat similar to results reported by Tang et al. [2003] and Ambrose et al. [1996] for thin films of $\mathrm{CoO}$, although they offered slightly different explanations. Tang et al. showed that the Néel temperature of the $\mathrm{CoO}$ layers decreases slightly with decreasing $\mathrm{CoO}$ layer thickness from 100 to $30 \AA$, but then exhibits a sharp decrease for $\mathrm{CoO}$ layer thickness below $20 \AA$. They suggested that this dramatic decrease below $20 \AA$ is due to the structural change of $\mathrm{CoO}$ from crystalline to 
amorphous rather than a finite size effect. And this structural change also leads to an increased number of weakly coupled uncompensated spins causing a significant change in temperature dependence of the magnetization. Whereas Ambrose et al explained the decrease of $T_{N}$ for thin films of $\mathrm{CoO}$ in terms of a finite size effect, where $T_{N}$ was found to decrease as

$$
\left\{\mathrm{T}_{\mathrm{N}}(\infty)-\mathrm{T}_{\mathrm{N}}(\mathrm{t})\right\} / \mathrm{T}_{\mathrm{N}}(\infty)=\left(\xi_{0} / \mathrm{t}\right)^{\lambda}
$$

where $\xi_{0}$ is the extrapolated correlation length at $\mathrm{T}=0 \mathrm{~K}$ and $\lambda=1 / v$ is the shift exponent for the finite-size scaling as the thickness of the film is lowered ( $v=2 / 3$ for $3 \mathrm{~d}$ systems).

In NiO NP, our TEM studies show the morphology for the smaller particles to be rod-like, changing over to nearly spherical shapes for $\mathrm{D}>10 \mathrm{~nm}$. Thus this structural change can also be the reason for the sharp decrease of $\mathrm{NiO} \mathrm{T}_{\mathrm{N}}$ below $10 \mathrm{~nm}$. The fitted curve for the parameters, $\xi_{0}=3.17(0.2) \mathrm{nm}$ and $\lambda=3.24$ (0.5), is also shown in Fig. 6.12, suggesting $v \approx 1 / 3$ for our NiO-NP system, although the limited number of data points makes it difficult to more accurately determine $\lambda=1 / v$. 
Table 6.1: Calculated values of the $\mathrm{Ni}^{2+}$ spins for a sphere of diameter $\mathrm{D}: \mathrm{n}_{\mathrm{A}}=\#$ spins on the surface layer; $n_{D}=$ total number of spins; $n_{C}=$ number of spins contributing to $\mu_{P}$ evaluated from the Langevin fits. Also listed are the ratios $\mathrm{n}_{\mathrm{C}} / \mathrm{n}_{\mathrm{A}}$ and $\mathrm{n}_{\mathrm{C}} / \mathrm{n}_{\mathrm{D}}$.

\begin{tabular}{|c|c|c|c|c|c|c|}
\hline $\mathrm{D}(\mathrm{nm})$ & $\mathrm{n}_{\mathrm{A}}$ & $\mathrm{n}_{\mathrm{D}}$ & $\begin{array}{c}\mu_{\mathrm{P}} / \mu_{\mathrm{B}} \\
\text { (uncoated) }\end{array}$ & $\begin{array}{c}\left(\mu_{\mathrm{P}} / \mu_{\mathrm{B}}\right) / 2.2 \\
=\mathrm{n}_{\mathrm{C}}\end{array}$ & $\mathrm{n}_{\mathrm{C}} / \mathrm{n}_{\mathrm{A}}$ & $\mathrm{n}_{\mathrm{C}} / \mathrm{n}_{\mathrm{D}}$ \\
\hline 5 & 468 & 3,586 & 1,250 & 568 & 1.21 & 0.158 \\
\hline 7 & 881 & 9,840 & 1,115 & 507 & 0.58 & 0.051 \\
\hline 8 & 1,151 & 14,689 & 1,250 & 568 & 0.49 & 0.039 \\
\hline 12 & 2,590 & 49,577 & 1,780 & 809 & 0.31 & 0.016 \\
\hline 16 & 4,640 & 117,516 & 2,500 & 1,136 & 0.24 & 0.010 \\
\hline 20 & 7,193 & 229,523 & 3,000 & 1,364 & 0.19 & 0.006 \\
\hline $\mathrm{D}(\mathrm{nm})$ & $\mathrm{n}_{\mathrm{A}}$ & $\mathrm{n}_{\mathrm{D}}$ & $\begin{array}{c}\mu_{\mathrm{P}} / \mu_{\mathrm{B}} \\
\text { (coated) }\end{array}$ & $\begin{array}{c}\left(\mu_{\mathrm{P}} / \mu_{\mathrm{B}}\right) / 2.2 \\
=\mathrm{n}_{\mathrm{C}}\end{array}$ & $\mathrm{n}_{\mathrm{C}} / \mathrm{n}_{\mathrm{A}}$ & $\mathrm{n}_{\mathrm{C}} / \mathrm{n}_{\mathrm{D}}$ \\
\hline 5 & 468 & 3,586 & 1,000 & 455 & 1.03 & 0.13 \\
\hline 7 & 881 & 9,840 & 900 & 409 & 0.46 & 0.042 \\
\hline 11 & 2,176 & 38,187 & 1,500 & 682 & 0.31 & 0.018 \\
\hline 17 & 5,197 & 140,956 & 4,000 & 1,818 & 0.35 & 0.013 \\
\hline 27 & 13,110 & 564,712 & 8,000 & 3,636 & 0.28 & 0.006 \\
\hline
\end{tabular}




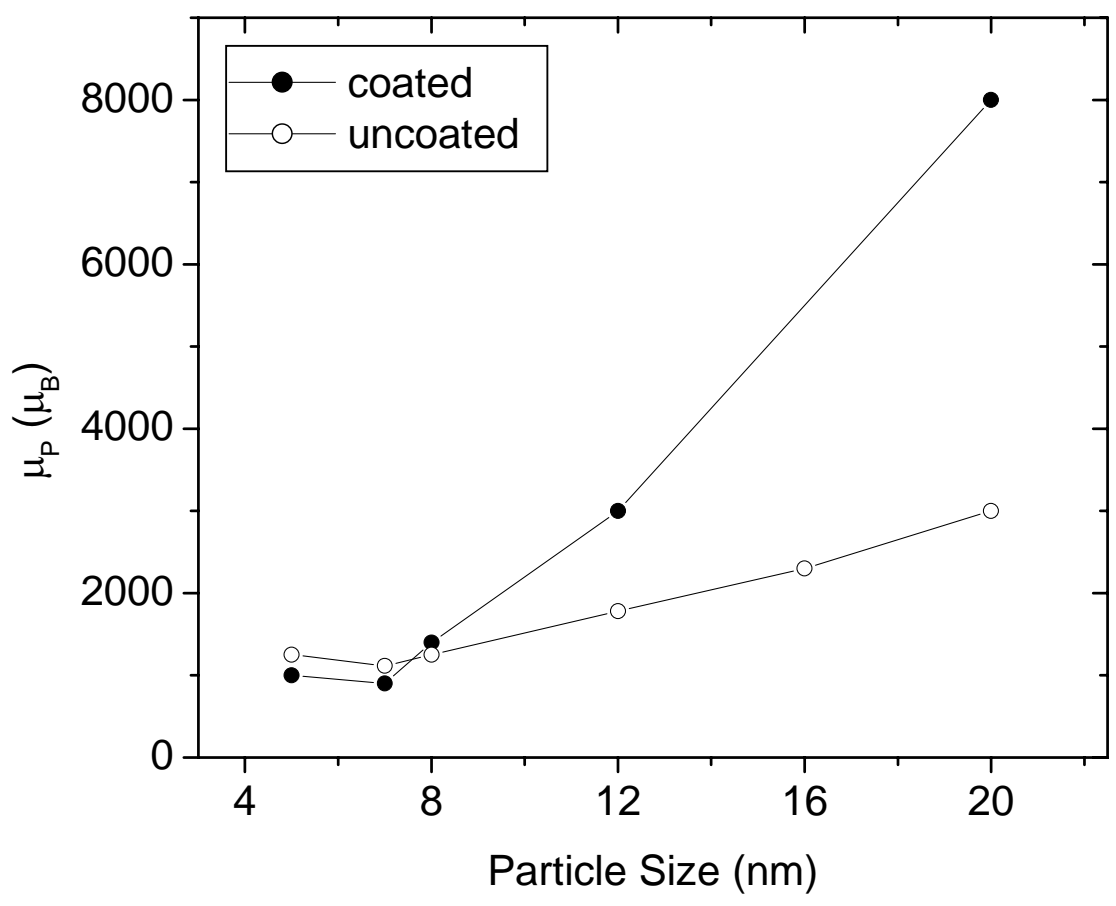

Fig. 6.10 Plot of $\mu_{P}$ vs. particle size D shows that $\mu_{P}$ increases with increase in D. 


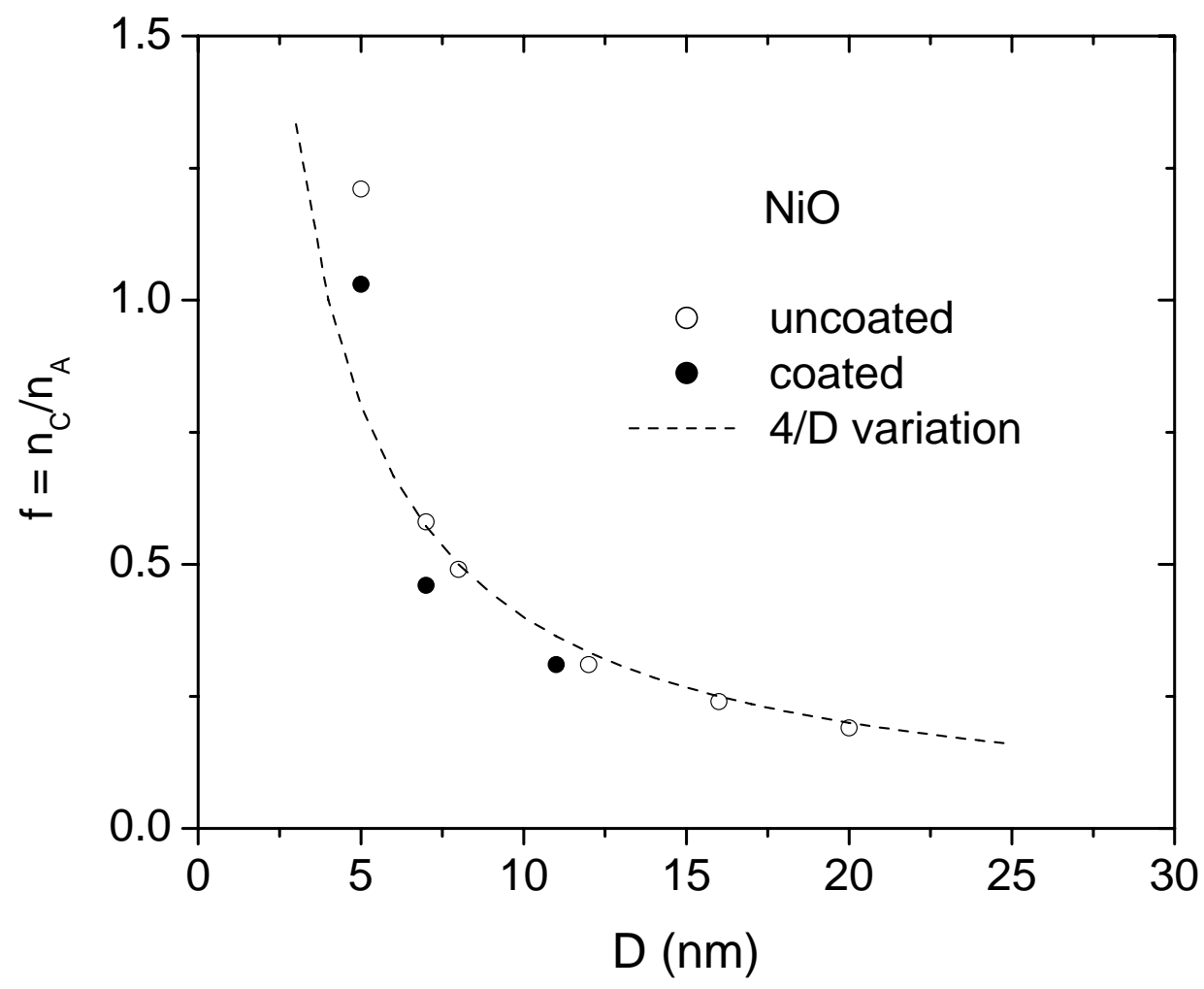

Fig. 6.11 Plot of the fraction $\mathrm{f}$ of the surface layer spins contributing to $\mu_{\mathrm{P}}$ against $\mathrm{D}$ for the coated and uncoated NiO-NP. The dashed line is the fitted 1/D variation for the uncoated particles only. 


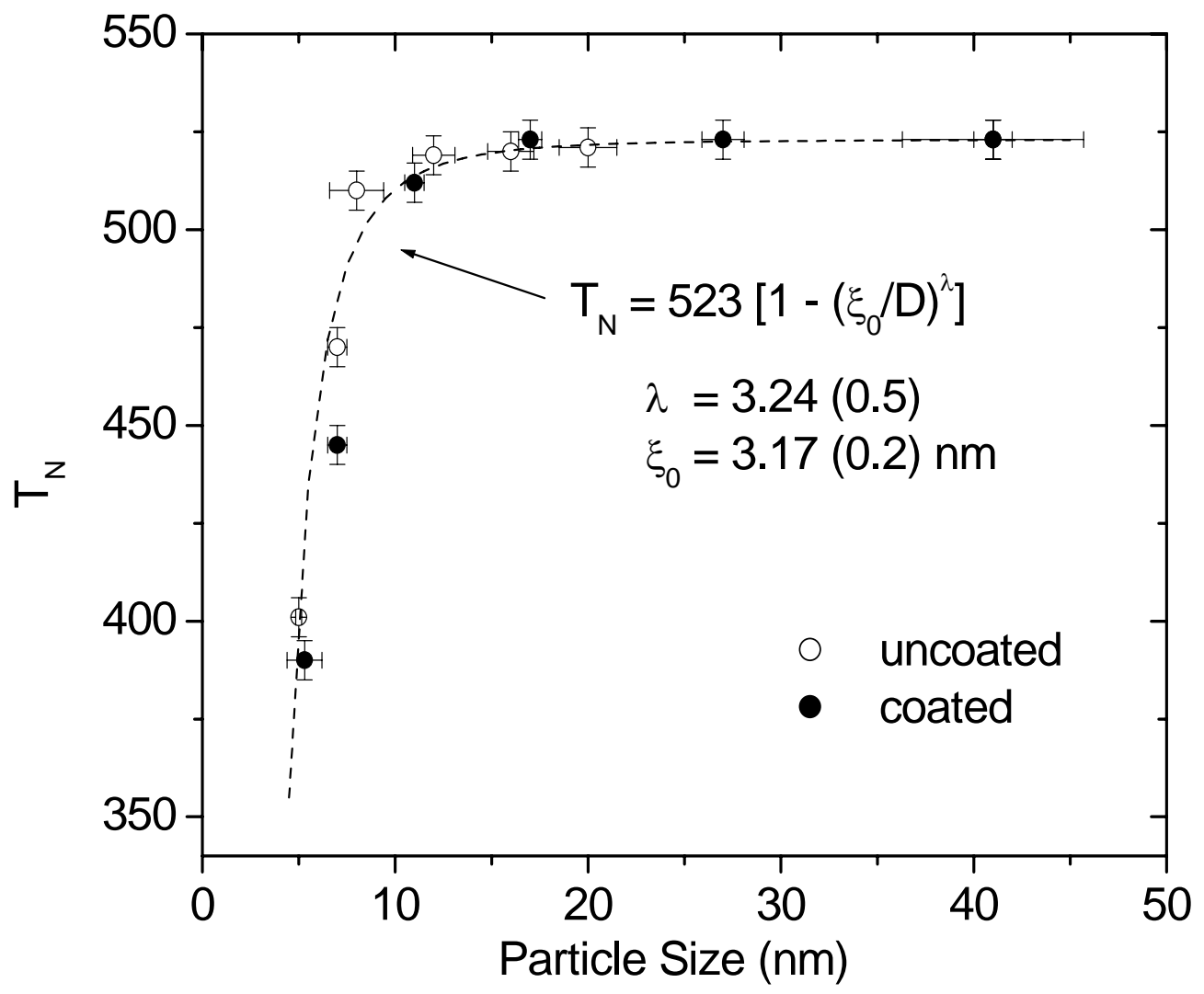

Fig. 6.12 Plots of estimated $\mathrm{T}_{\mathrm{N}}$ (Fig. 6.7) against particle size $\mathrm{D}$ for the coated and uncoated NiO-NP. The dashed line is a theoretical fit with the parameters given in the text. 


\subsection{Hysteresis loop measurements}

We performed the measurements of the hysteresis loops for all the particles. Following the procedure as described in section 5.4, the hysteresis loops were measured at $5 \mathrm{~K}$ using $\mathrm{ZFC}$ and $\mathrm{FC}$ cases up to $\mathrm{H}= \pm 50 \mathrm{kOe}$. For the $\mathrm{FC}$ case, the samples were cooled from $390 \mathrm{~K}$ to $5 \mathrm{~K}$ in $\mathrm{H}=20 \mathrm{kOe}$, followed by the hysteresis loop measurements. The loops for each particle are shown in Fig. 6.13 for the uncoated particles and in Fig. 6.14 for the coated particles. The hysteresis loop parameters, viz., coercivity $\mathrm{H}_{\mathrm{C}}$ and exchange bias $\mathrm{H}_{\mathrm{E}}$ for both the uncoated and coated particles are summarized in Table 6.2.

For all the particles, $\mathrm{H}_{\mathrm{E}}$ values are larger for the $\mathrm{FC}$ measurement than the ZFC. For the uncoated samples, the loops are narrow for the smaller particles but the loops are larger for the larger particles leading to increase in the values of $\mathrm{H}_{\mathrm{E}}$ and $\mathrm{H}_{\mathrm{C}}$. However, for the coated samples, the loops are narrow even in $27 \mathrm{~nm}$ particles. This is an interesting result since several hysteresis loops measurements for AF-NP [Makhlouf et al. 1997a and 1997b, Kodama et al, 1999, and Punnoose et al. 2001] show that most AF-NPs below their blocking temperatures show significant increase in the loop width with strong exchange bias when field-coolded from $\mathrm{T}>\mathrm{T}_{\mathrm{P}}$. The magnitudes of $\mathrm{H}_{\mathrm{C}}$ for the coated samples decrease because of the absence of interparticle interaction.

An interesting result observed here is the appearance of steps in the hysteresis loops in the low-field region for the uncoated particles. These step-like features are dramatically reduced by increasing particle size. Such steps in the hysteresis loops have been observed for Ferrihydrite NP [Punnoose et al. 2004] and for single-domain Ni nanomagnets [Grundler et al. 1999]. The recent theoretical studies of Fraerman and Sapozhnikov [2002] for one dimensional NP system, explained that the nature of the steps is determined by the competing effects of coercivity, interparticle interactions, and thermal energy. Punnoose et al. explained that the steps in the hysteresis loops in the low-field region could be due to the dipolar interparticle interaction. Our data support their interpretation from the reduction of the step-like features for our coated samples compared to the uncoated samples.

To explain the hysteresis loops for NiO-NP, more experiments are needed,

especially temperature dependence of the loops parameters below the blocking temperatures with the change in particle size. 


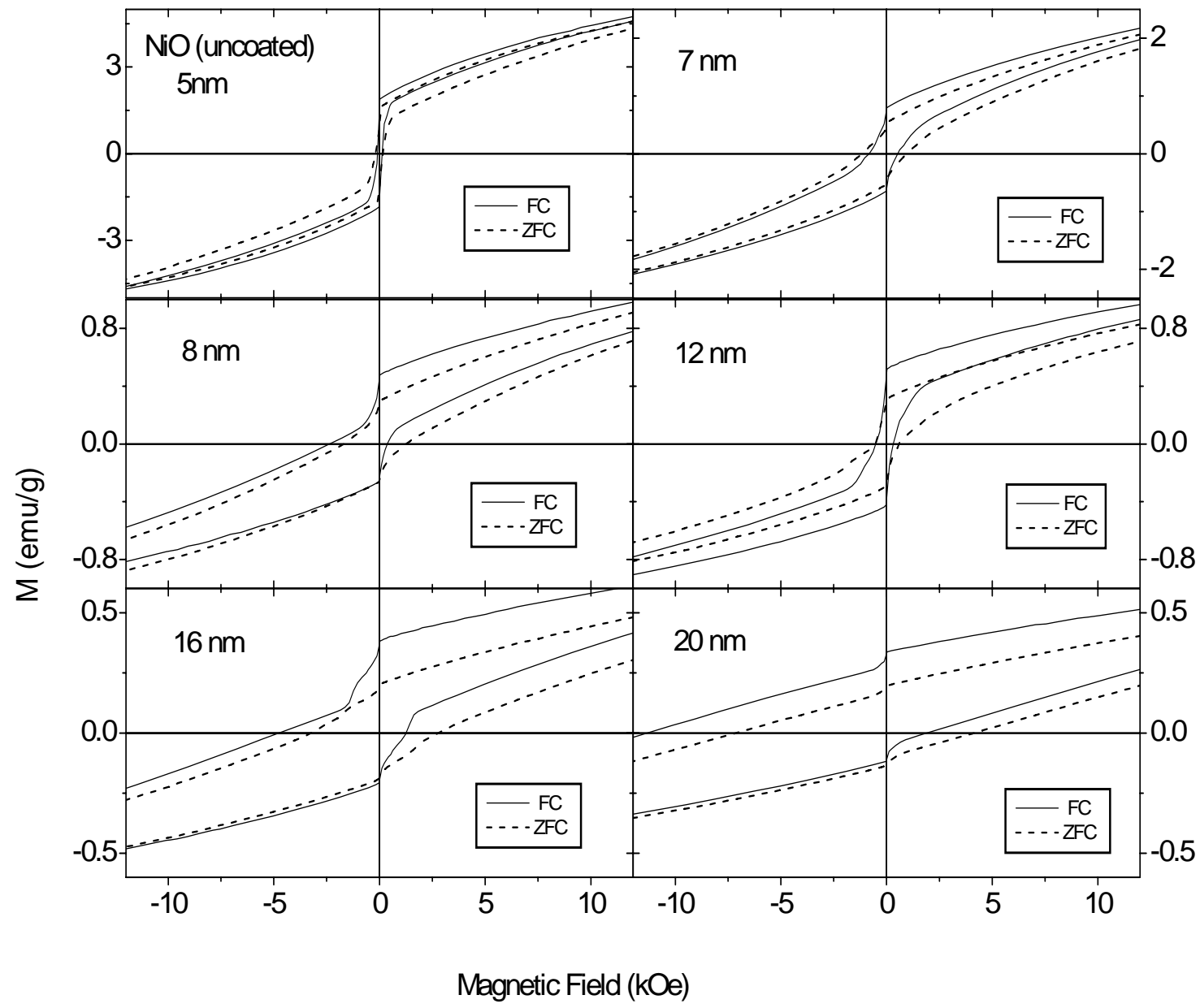

Fig. 6.13 Hysteresis loops for the various particle sizes are measured in zero - field cooled (ZFC) and field cooled (FC) cases at $\mathrm{T}=5 \mathrm{~K}$ for uncoated. 


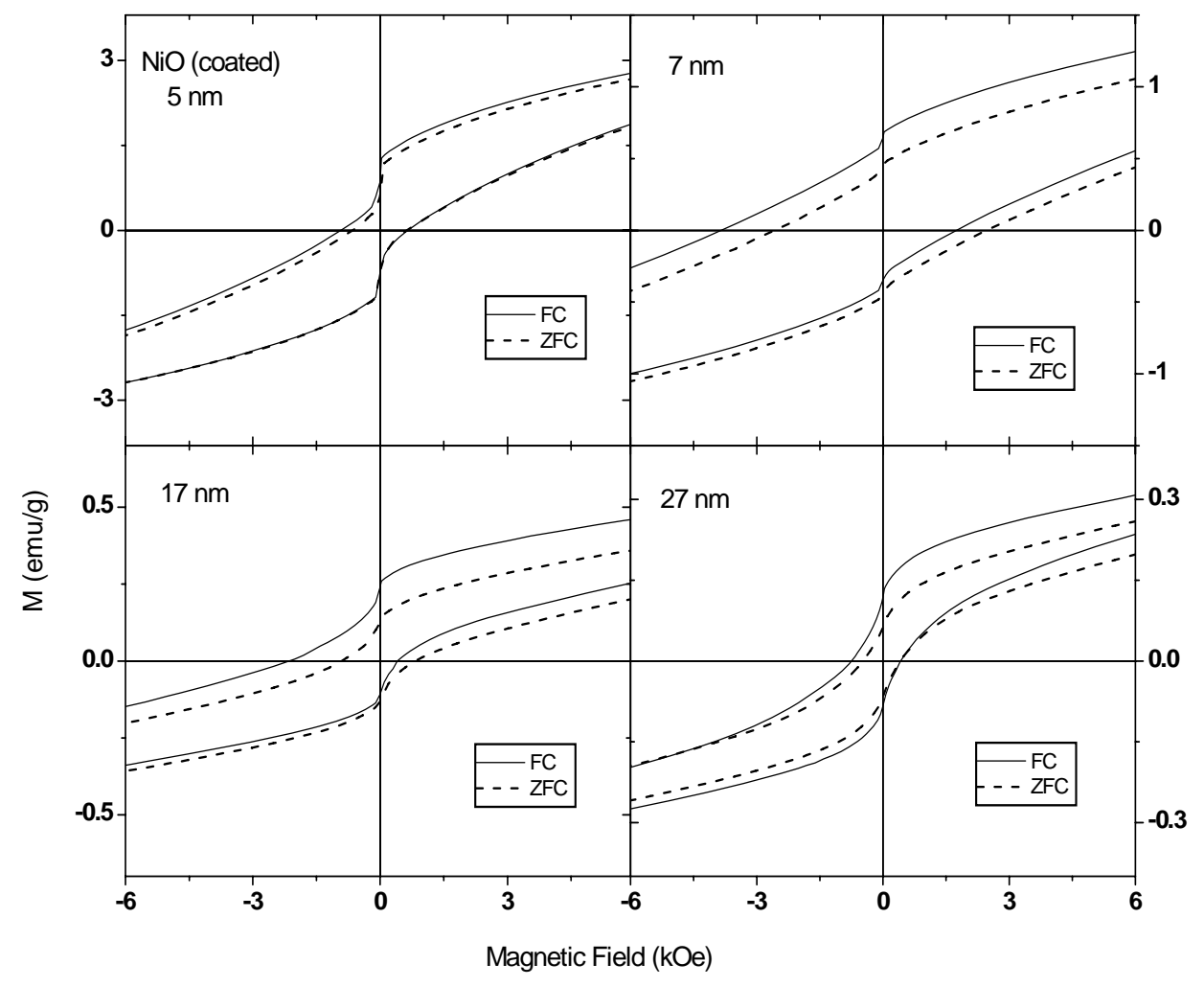

Fig. 6.14 Hysteresis loops for the various particle sizes are measured in zero - field cooled (ZFC) and field cooled (FC) cases at $\mathrm{T}=5 \mathrm{~K}$ for coated samples. 


\begin{tabular}{|c|c|c|c|c|c|c|c|}
\hline \multicolumn{2}{|c|}{$(\mathrm{ZFC})$} & $5 \mathrm{~nm}$ & $7 \mathrm{~nm}$ & $8 \mathrm{~nm}$ & $12 \mathrm{~nm}$ & $16 \mathrm{~nm}$ & $20 \mathrm{~nm}$ \\
\hline \multirow{2}{*}{$-\mathrm{H}_{\mathrm{E}}$} & Uncoated & 0 & 85 & 235 & 50 & 250 & 1,470 \\
\hline & Coated & 0 & 50 & 0 & 40 & - & 30 \\
\hline \multirow{2}{*}{$\mathrm{H}_{\mathrm{C}}$} & Uncoated & 115 & 1,030 & 1,490 & 560 & 3.020 & 5,610 \\
\hline & Coated & 640 & 2,510 & 600 & 860 & - & 440 \\
\hline \multicolumn{2}{|c|}{$(\mathrm{FC})$} & $5 \mathrm{~nm}$ & $7 \mathrm{~nm}$ & $8 \mathrm{~nm}$ & $12 \mathrm{~nm}$ & $16 \mathrm{~nm}$ & $20 \mathrm{~nm}$ \\
\hline \multirow{2}{*}{$-\mathrm{H}_{\mathrm{E}}$} & Uncoated & 0 & 210 & 1010 & 85 & 1750 & 4540 \\
\hline & Coated & 105 & 1070 & 170 & 855 & - & 160 \\
\hline \multirow{2}{*}{$\mathrm{H}_{\mathrm{C}}$} & Uncoated & 180 & 630 & 1,010 & 405 & 2,970 & 6,560 \\
\hline & Coated & 670 & 2790 & 460 & 1270 & - & 580 \\
\hline
\end{tabular}

Table 6.2 The hysteresis loop parameters observed in the ZFC and FC modes are summarized for each particle and here field unit is Oe. 


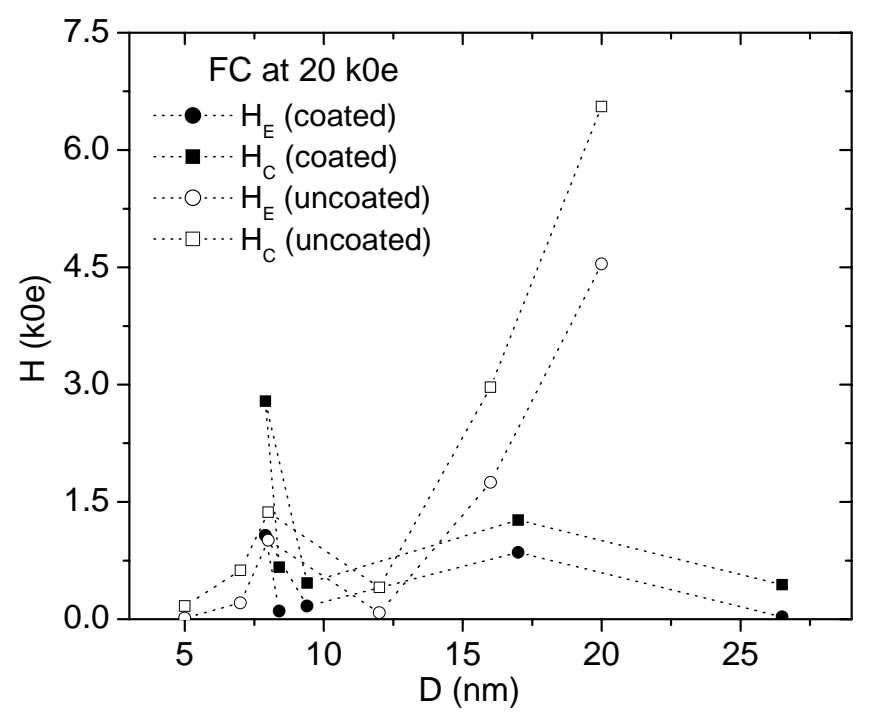

(a) Field - cooled

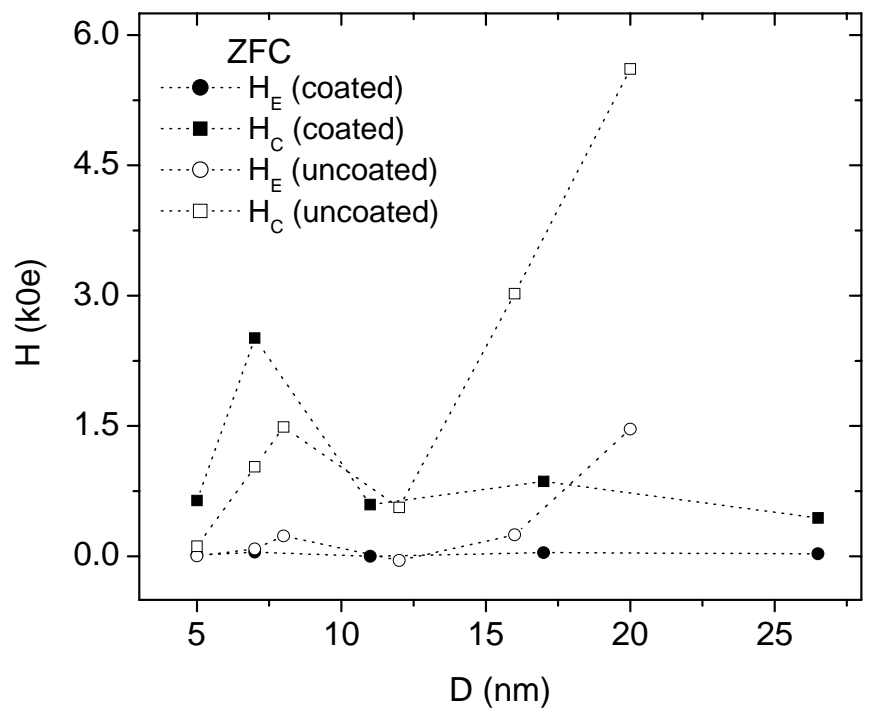

(b) Zero Field - cooled

Fig. 6.15 Plot of the variations of loop shift $\mathrm{H}_{\mathrm{E}}$ and coercivity $\mathrm{H}_{\mathrm{C}}$ on each particle size under FC and ZFC condition. 


\section{CHAPTER VII}

\section{CONCLUSION}

\subsection{Structure and Morphology of NiO-NP}

Studies reported in this dissertation have shown the changing morphology of the NiO-NP prepared by the sol-gel technique with increase in size D. For $\mathrm{D}<10 \mathrm{~nm}$, the particles have primarily rod-like features observed in TEM studies whereas for D $>10$ $\mathrm{nm}$ the rod-like features give way to more sphere shaped particles. However, for the smaller particles with nanorods features, it turns out that the volume of the nanorods is quite close to a sphere of diameter $\mathrm{D}$ determined by XRD so that XRD measures average diameter of an equivalent sphere. This comparison between XRD and TEM showed the importance of both TEM and XRD studies for investigating nanoparticles. The particles coated with oleic acid to reduce interparticle interaction show similar change in morphology with change in size.

The intensities (area under the peaks) of the XRD lines were analyzed in terms of the Debye-Waller factors B for the Ni and $\mathrm{O}$ atoms. A general trend of the increase of $\mathrm{B}$ with decrease in size $\mathrm{D}$ is observed suggesting that the atoms in the nanoparticles are more loosely bound than those in bulk materials.

\subsection{Effects of interparticle interaction on $5 \mathrm{~nm}$ NiO-NP}

The blocking temperatures $T_{B}$ are measured at different measuring frequencies $f_{m}$ by magnetization and EMR studies for both coated and uncoated $\mathrm{NiO} 5 \mathrm{~nm}$ nanorods. The dc and ac measurements show that blocking temperature $T_{B}$ decreases as field $H$ increases and increases as frequency $f$ increases, respectively.

The Vogel-Fulcher law describes well the magnetic relaxation of the $5 \mathrm{~nm}$ particles, $T_{B}$ being enhanced by $T_{0}$ in the uncoated particles. Also, it has been shown that $\mathrm{T}_{0}$ provides a good measure of the effect of interparticle interactions on magnetic relaxation. Oleic acid coating of $\mathrm{NiO} 5 \mathrm{~nm} \mathrm{NP}$ essentially eliminated these interparticle interactions. 


\subsection{Size dependence of the magnetic properties of NiO-NP}

The blocking temperature, $\mathrm{T}_{\mathrm{P}}$ for non-interacting particles is expected to be proportional to the particle volume $\mathrm{V}$ in line with the observations for the coated particles. However, for $\mathrm{D}<10 \mathrm{~nm}, \mathrm{~T}_{\mathrm{P}}$ for the uncoated particles continues to increase with decrease in $\mathrm{D}$ due to interparticle interaction.

Unlike previous studies on NiO-NP, we were able to fit the $\mathrm{M}$ vs. $\mathrm{H}$ data for $\mathrm{T}>$ $\mathrm{T}_{\mathrm{P}}$ for most sizes with the modified Langevin function and determine $\mu_{\mathrm{P}}$ and its (very weak) temperature dependence. Further analysis showed that $\mu_{\mathrm{P}}$ is determined by a fraction $f$ of the surface-layer spins, with $f$ varying as $1 / D$ such that for $D=5 \mathrm{~nm}$, all the $\mathrm{Ni}^{2+}$ in the surface layer contribute a moment of $2.2 \mu_{\mathrm{B}}$ to $\mu_{\mathrm{P}}$. Additionally, comparison of the data for the OA-coated and uncoated NP provided clear evidence for the presence (absence) of interparticle interaction in the uncoated (coated) nanoparticles. Finally, the decrease of the Néel temperature $\mathrm{T}_{\mathrm{N}}$ with decrease in $\mathrm{D}$, as expected for reduced dimensionality, was observed.

\subsection{Hysteresis loop measurements of $\mathrm{NiO}-\mathrm{NP}$}

We measured the hysteresis loops of $\mathrm{NiO}-\mathrm{NP}$ at $5 \mathrm{~K}$ below the blocking temperature. The step-like features observed in the low-field regions of the hysteresis loops might be due to interparticle interaction since the features are quiet reduced by OA coating. The temperature dependence of the hysteresis loop parameters (exchange bias, coercivity, remanence) for the different particles and the nature of the magnetic state for $\mathrm{T}<\mathrm{T}_{\mathrm{P}}$ requires additional studies. 


\section{REFERENCE:}

- Allia, P., Coisson, M., Tiberts, P., Vinai, F., Knobel, M., Novak, M.A., and Nunes, W.C., Ibid $\underline{64}, 144420$ (2001).

- Ambrose, T. and Chien, C.L., Phys. Rev. Lett, 76, 1743 (1996).

- Anderson, P.W., Phys. Rev. 115, 2 (1959).

- Barbier, A. et al, Phys. Rev. Lett. 93, 257208 (2004).

- Bartel, L.C. and Morosin, B., Phys. Rev. B 3 , 3 (1971).

- Bean, C.P. and Livingston, J.D., J. Appl. Phys. $\underline{30,} 1205$ (1959).

- Berkowitz, A.E., Takano, K., J. Magn. Magn. Mater. 200, 552 (1999).

- Bødker, F., Hansen, M.F., Koch, C.Bender, and Mørup, S., J. Magn. Magn. Mater. $\underline{221}, 32(2000)$.

- Brown, W.F., Phys. Rev. 130, 1677 (1963).

- Castner, T.G. and Seehra, M.S., Phys. Rev. B 4, 38 (1971) and references therein.

- Chantrell, R.W., Walmsley, N., Gore, J. and Maylin, M., Phys. Rev. B $\underline{63}, 024410$ (2000).

- Chien, C.L., J. Appl Phys, 695267 (1991).

- Cullity, B.D., the book of "Introduction to Magnetic Materials.", Addison-Wesley Publishing Company (1972).

- Denardin, J.C., Brandl, A.L., Knobel, M., Panissod, P., Pakhomov, A.B., Liu, H., and Zhang, X.X., Phys. Rev. B $\underline{65}, 064422$ (2002).

- Dick, B. and Overhauser, A., Phys. Rev. 112, 90-103 (1958).

- Dickson, D.P.E., Reid, N.M.K., Hunt, C., Williams, H.D., El-Hilo, M., and O’Grady, K., J. Magn. Magn. Mater. 125, 345 (1993).

- Dietz, R.E., Parisot, G.I., and Meixner, A.E., Phys. Rev. B̧ㅡ, 2302 (1971).

- Dormann, J.L. and Fiorani, D. (editors), Magnetic properties of Fine Particles (North- Holland, Amsterdam 1992).

- Dormann, J.L., Bessais, L., and Fioani, D., J. Phys.C: Solid State Phys. 21, 2015 (1988).

- Dormann, J.L., D'Orazio, F., Lucari, F., Tronc, E., Prené, P., Jolivet, J.P., Fiorani, D., Cherkaoui, R., and Noguès, M., Phys. Rev. B 포, 14291 (1996). 
- Dormann, J.L., Fiorani, D., and Yamani, M.El., Phys. Lett. A 120, 95 (1987).

- Dormann, J.L., Fiorani, D., Tholence, J. L., and Sella, C., J. Magn. Magn. Mater. $\underline{40}, 1$ (1983).

- Dromann, J.L., Gibart, P., Suran, G., Tholence, J.L., and Sella, C., J. Magn. Magn. Mater. 15- $\underline{18}, 1121$ (1980).

- El-Hilo, M., O`Grady, K., and Chantrell, R.W., J. Magn. Magn. Mater. 114, 307 (1992).

- El-Hilo, M., O’Grady, K., and Chantrell, R.W., J. Magn. Magn. Mater. 117, 21 (1992).

- Fiorani, D., Dormann, J.L., Tholence, J.L., Bessaïs, L., and Villers, G., J. Magn. Magn. Mater. 54-57, 173 (1986).

- Fiorani, D., Tholence, J.L., and Dormann, J.L., J. Magn. Magn. Mater. 31-34, 947, (1983).

- Fiorani, D., Tholence, J.L., and Dormann, J.L., Physica B 107, 64 (1981).

- Fraerman, A.A. and Sapozhnikov, M.V., Phys. Rev. B $\underline{65}$, 184433 (2002).

- Gittleman, J.I., Abeles, B., and Bozoeski, S., Phys. Rev. B9, 3891 (1974).

- Gonzalez - Miranda, J.M. and Tejada, J., Phys. Rev. B $\underline{49}, 6$ (1994).

- Grundler, D., Meier, G., Broocks, K.B., Heyn, C., and Heitmann, D., J. Appl. Phys. $\underline{85}, 6175$ (1999).

- Harris, J.G.E., Grimaldi, J.E., Awschalom, D.D., Chilolero, A., Loss, D., Phys. Rev. B $\underline{60}, 3453$ (1999).

- Hutchings, M.T. and Samuelsen, E.J., Phys. Rev. B $\underline{6}, 9$ (1972).

- Ibrahim, M.M., Darwish, S.D., and Seehra, M.S., Phys. Rev. B $\underline{1}$, 2955 (1995).

- Jacobs, I.S. and Bean, C.P., Magnetism, Vol III, 294 (1963).

- Jonsson, T., Nordblad, P., and Svedlindh, P., Phys. Rev. B 므, 497 (1998).

- Kechrakos, D. and Trohidou, K.N., Phys Rev. B 타, 12169 (1998).

- Kenning, G.G., Slaughter, J.M., Cowen, J.A., Phys. Rev. Lett. 59, 2596 (1987).

- Kilcoyne, S.H. and Cywinski, R., J. Magn. Magn. Mater. 140-144, 1466 (1995).

- Klug, H.P. and Alexander, L.E., book of “X - Ray Diffraction Procedures.”, (2 ${ }^{\text {nd }}$ edition, Wiley - Interscience 1974). 
- Kodama, R.H. and Berkowitz, A.E., Phys. Rev. B 599, 6321 (1999).

- Kodama, R.H., Makhouf, S.A., and Berkowitz, A.E., Phys. Rev. Lett. 79, 1393 (1997).

- Koksharov, Yu. A. et al, Phys. Rev. B $\underline{63}, 012407$ (2000).

- Kwon, S.K. and Min, B.I., Phys. Rev. B 62 , 73 (2000).

- Makhlouf, S.A., Parker, F.T., and Berkowitz, A.E., Phys. Rev. B 55, R14717 (1997).

- Makhlouf, S.A., Parker, F.T., Spada, F.E., and Berkowitz, A.E., J. Appl. Phys. $\underline{\text { 81, }}$ 5561 (1997).

- Makhlouf, S.A., Parker, F.T., Spada, F.E., and Berkowitz, A.E., J. Appl. Phys. $\underline{\text { 81, }}$ 5561 (1997).

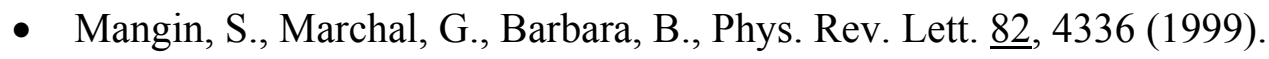

- Martínez, B., Obradors, X., Balcells, L., Rouanet, A., and Monty, C., Phys. Rev. Lett. $\underline{80}, 181-184$ (1998).

- Milano, J., Steren, L.B., and Grimsditch, M., Phys. Rev. Lett. 93, 077601 (2004).

- Mocuta et al. Phys. Rev. B $\underline{68}, 14416$ (2003).

- Morrish, Allan H., “The Physical Principles of Magnetism.”, IEEE, (2001).

- Mørup, S. and Fandsen, C., Phys. Rev. Lett. 92, 217201 (2004).

- Mørup, S., Europhys. Lett. 28, 671 (1994).

- Nagata, K., Ishihara, A., J. Magn. Magn. Mater. 104-107, 1571 (1992).

- Néel, L., Compt. Reud. 252, 4075 (1961); Néel L in Low Temperature Physics, edited by DeWitt C, Dreyfus B, and DeGeunes P.G (Gordon and Breach, London, 1962), p. 411.

- Neubeck, W., Vettier, C., Fernandez, V., Bergevin, F. de, and Giles, C., J. Appl. Phys. $\underline{85}, 4847$ (1999).

- Ohldag, H. et al. Phys. Rev. Lett. 6ㅜ, 2878 (2001).

- Owen, J. and Thornley, J.H.M., Rept. Progr. Phys. 29, 675 (1966).

- Pierre, A.C., "Introduction to Sol-Gel Processing.", (Kluwer Academic Publishers, Norwell, MA, 1998). 
- Poole, C.P. and Owens, F.J., "Introduction to Nanotechnology.", (Wiley Publishers, 2000).

- $\quad$ Poole, Jr. C.P., Electron Spin Resonance, $2^{\text {nd }}$ ed., p. 483 (Dover, New York, 1983).

- Punnoose, A., Phanthavady, T., Seehra, M.S., Shah, N.P., and Huffman, G.P., Phys. Rev. B $\underline{69}, 054425$ (2004).

- Punnoose, A., Seehra, M.S., Tol, J.Van., and Brunel, L.C., J. Magn. Magn. Mater. $\underline{288}, 168$ (2005).

- Resnick, D.A., Gilmore, K., Idzerda, Y.U., Klem, M.T., Allen, M., Douglas, T., Arenholz, E., and Young, M., J. Appl. Phys. 99, 08Q501 (2006).

- Richardson, J.T. and Milligan, W.O., Phys. Rev. 102, 1289 (1956).

- Richardson, J.T., Yiagas, D.I., Turk, B., Forster, K., and Twigg, M.V., J. Appl. Phys. $\underline{70}, 6977$ (1991).

- $\quad$ Roth, W., J. Appl. Phys. $\underline{31,} 2000$ (1960).

- Rubinstein, M., Kodama, R.H., and Makhlouf, S.A., J. Magn. Magn. Magn. 234, 289 (2001).

- Sako, S., Ohshima, K., J. Phys. Soc. Japan 64, 944 (1995).

- Samuel, Smart J., in Effective Field Theories of Mangetism, (Saunders, W.B. Company, Philadelphia \& London 1966).

- See various papers in Magnetic properties of Fine Particles, edited by Dormann, J.L. and Fiorani, D., (Elsevier Science Amsterdam, 1992); Nanophase Materials: Synthesis, Properties, Applications, edited by Hadjipanayis, G.C. and Siegel, R.W., (Kluwer, Dordrecht, 1994).

- Seehra, M.S. and Castner, T.G., Phys. Rev. B 1 , 2289 (1970); Solid. St. Commun. $\underline{8}, 787$ (1970).

- Seehra, M.S., and Giebultowicz, T.M., Phys. Rev. B $\underline{38}, 11898$ (1988).

- Seehra, M.S., Dutta, P., Shim, H., and Manivannan, A., Solid St. Commun. 129, 721 (2004).

- Seehra, M.S., Punnoose, A., Roy, P., and Manivannan, A., IEEE Trans. Magn. $\underline{37}$, 2007 (2001). 
- Seehra, M.S., Shim, H., Dutta, P., Manivannan, A., and Bonevich, J., J. Appl. Phys. 97, $10 \mathrm{~J} 609$ (2005).

- Shim, H., Manivannan, A., Seehra, M.S., Reddy, K.M., and Punnoose, A., J. Appl. Phys. 99, 08Q503 (2006).

- Shtrikman, S. and Wohlfarth, E.P., Phys. Lett. $\underline{\text { 85A, }} 467$ (1981).

- Shull, C.G., Strauser, W.A., and Wollan, E.O., Phys. Rev. 푸, 333 (1951).

- Silva, N.J.O., Amaral, V.S., and Carlos, L.D., Phys. Rev. B $\underline{71}, 184408$ (2005).

- Smart, J.S., in “Effective Field Theories of Magnetism.” (1996).

- $\quad$ Speliotis, D.E., J. Magn. Magn. Mater. 193, 29 (1999).

- Srinivasan, G. and Seehra, M.S., Phys. Rev. B29, 11 (1984).

- Stoner, E.C. and Wohlfarth, E.P., Phil. Trans. Roy. Soc. (London) A - 240, 599 (1948).

- Street, R. and Wooley, J.C., Proc. Phys. Soc. London A $\underline{62}, 562$ (1949).

- Tang, Y.J., Smith, David J., Zink, B.L., Hellman, F., and Berkowitz, A.E., Phys. Rev. B $\underline{67}, 054408$ (2003).

- $\quad$ Tang, Z.X., Sorensen, C.M., Klabunde, K.F., Hadjipanayis, G.C., Phys. Rev. Lett. $\underline{25}, 3602$ (1991).

- $\quad$ Tiwari, S.D. and Rajeev, K.P., Phys. Rev. B $\underline{72}, 104433$ (2005).

- Vargas, J.M., Socolovsky, L.M., Knobel, M., and Zanchet, D., Nanotechnology $\underline{16}, 5285$ (2005).

- Vincent, E., Yuan, Y., Hammann, J., Hurdequint, H., and Guevara, F., J. Magn. Magn. Mater. 161, 209 (1996).

- Vollath, D., Szabó, D.V., Willis, J.O., Mater. Lett. 29, 271 (1996).

- Voskoboynik, U., Acta Phys. Pol. A $\underline{92}$ Supplement, S $\underline{43}$ (1997).

- Warren, B.E., in “X-Ray Diffraction.” (Dover Publication, N.Y., 1996).

- Weiss, P., J. Phys. $\underline{6}, 667$ (1907).

- Wenger, L.E. and Mydosh, J.A., Phys. Rev. B 29, 4156 (1984).

- Zhang, J., Boyd, C., and Luo, W., Phys. Rev. Lett. 77, 390 (1996). 